\title{
Polarizable Multipolar Molecular Dynamics Using Distributed Point Charges
}

\author{
Mike Devereux, ${ }^{\dagger}$ Marco Pezzella, ${ }^{\dagger}$ Shampa Raghunathan, ${ }^{\dagger}$ and Markus \\ Meuwly,,$+ \ddagger$ \\ $\dagger$ Department of Chemistry, University of Basel, Klingelbergstrasse 80, CH-4056 Basel, \\ Switzerland \\ $\ddagger$ Department of Chemistry, Brown University, Providence, RI, USA \\ E-mail: m.meuwly@unibas.ch
}

\begin{abstract}
Distributed point charge models (DCM) and their minimal variants (MDCM) have been integrated with tools widely used for condensed-phase simulations, including a virial-based barostat and a slow-growth algorithm for thermodynamic integration. Minimal DCM is further developed with a systematic approach to reduce fitting errors in the electrostatic interaction energy and a new fragment-based approach offers considerable speedup of the MDCM fitting process for larger molecules with increased numbers of off-centered charged sites. Finally, polarizable (M)DCM is also introduced in the present work. The developments are used in condensed-phase simulations of popular force fields with commonly applied simulation conditions. (M)DCM equivalents for a range of widely used water force fields and for fluorobenzene $(\mathrm{PhF})$ are developed and applied along with the original models to evaluate the impact of reformulating the electrostatic term. Comparisons of the molecular electrostatic potential (MEP), electrostatic interaction energies, and bulk properties from molecular dynamics simulations for a range of models from simple TIP $n \mathrm{P}(n=3-5)$ to the polarizable, multipolar
\end{abstract}


iAMOEBA models for water and an existing quadrupolar model for PhF confirm that DCMs retain the accuracy of the original models, providing a homogeneous, efficient, and generic point charge alternative to a multipolar electrostatic model for force field development and multilevel simulations.

\section{INTRODUCTION}

Empirical force fields (FFs) are routinely used for simulating a multitude of chemical and biochemical phenomena. ${ }^{1-5}$ Commonly employed FFs divide interactions into intra- and intermolecular terms that include point charges (PCs) for Coulomb interactions. While interactions between nuclear-centered point charges are rapid to evaluate which allows application to large condensed-phase systems and relatively long timescales, their accuracy is compromised as they do not correctly describe charge density anisotropy. ${ }^{6}$ A new generation of FFs aims to overcome these drawbacks, either using higher-order multipolar electrostatics $^{7,8}$ in methods such as AMOEBA (atomic multipole optimized energetics for biomolecular applications), ${ }^{9-12}$ SIBFA (sum of interaction between fragments ab initio computed) ${ }^{13-17}$ and QCTFF (Quantum Chemical Topological Force Field), ${ }^{18}$ or using Gaussian functions to directly describe the underlying charge density in methods such as EFP (effective fragment potential), ${ }^{19}$ GEM (gaussian electrostatic model), ${ }^{20,21}$ and NEMO (nonempirical molecular orbital). ${ }^{22}$ On the other hand, the use of higher-order atomic multipoles, while resulting in improved accuracy, introduces non-negligible computational overhead due to the additional complexity and increased number of terms that need to be evaluated. ${ }^{23-26}$

An alternate tractable approach is to represent the MEP as a truncated multipole expansion transformed into a set of appropriately distributed point charges. Charges can be placed in fixed arrangements relative to the nuclei, ${ }^{26}$ or machine learning can be used to

replace fixed arrangements with a minimal set of optimally positioned off-center charges. ${ }^{27}$ Recently it was demonstrated that these Distributed Charge Models (DCMs) ${ }^{26}$ and Minimal 
Distributed Charge Models (MDCMs) ${ }^{27}$ can be implemented into widely used molecular dynamics software as an alternative to conventional, PC-based energy terms. The use of point charges for representing charge density anisotropy reduces the complexity of Coulomb terms relative to a traditional multipolar formalism considerably, allowing efficient MD simulations while maintaining the accuracy of a truncated multipole expansion.

The versatility of (M)DCMs (i.e. distributed charge models with and without machine learning optimization) additionally yields a homogeneous implementation of different types of electrostatic models (nuclear-centered charges, off-center charges and multipolar electrostatics) using a single routine, with combinations of models of different complexities in a single simulation - so-called 'multilevel' simulations. Its compatibility with remaining standard bonded and non-bonded FF terms promises to make adaptation of next-generation (multipolar) FF electrostatics straightforward in widely used simulation packages. ${ }^{1,3,28,29}$

The ability to generate models of increasing accuracy by increasing the number of charges in an MDCM fit offers an important tool to force field developers to carefully balance the accuracy of a model with the computational cost incurred from adding each additional charge. Models for the moiety or moieties of interest, such as a solute or protein ligand and immediate environment, can be created at the highest level of detail, while remaining interacting species can be optimally fitted to balance accuracy in the potential energy surface with computational efficiency to reach the system sizes and timescales required to sufficiently sample the relevant phase space. This multilevel approach is akin to the more familiar mixed quantum mechanical/molecular mechanical (QM/MM) treatments which also employ methods of different accuracy for different parts of a simulation system. ${ }^{30}$ Similarly, promising new models can equally be incorporated from one force field into another by transferring their parameters, and by refitting parameters of interacting neighbors at a level of detail that works optimally with that model, rather than combining existing models of different complexities 
that may not be compatible.

In this work recent advances in the implementation of (M)DCM are exploited and applied to condensed-phase simulations, including an intermediate fragment fitting step to improve efficiency of the MDCM fitting process for larger systems, improved error handling based on analysis of the relationship between errors in the fitted MEP and errors in the electrostatic interaction energy, integration with barostats for simulations in the isothermal-isobaric ensemble and slow-growth routines for thermodynamic integration calculations. Different (M)DCM models are developed to replace the electrostatic terms in several water force fields commonly used in chemical and biomolecular simulations and in a multipolar force field for PhF. In the first section of the results (M)DCM representations are generated for water models of increasing complexity ranging from the simple but widely used "TIP3P" 31 to the multipolar, polarizable "iAMOEBA" potential. ${ }^{32}$ All terms of each original force field other than the electrostatic term are left untouched, requiring particularly close agreement with the original charge model to avoid reparametrization. Then, a similar approach is taken for the PhF molecule, demonstrating the applicability to a solute molecule in a condensed-phase aqueous environment. Comparisons of energies and bulk properties in each case are used to demonstrate the accuracy of a distributed charge approach with respect to a more computationally complex multipolar description of molecular electrostatics.

\section{Background}

\section{$2.1 \quad \mathrm{DCM}$}

Multipolar force fields are based on the fact that any charge distribution can be represented as a series expansion, where the successive terms are multipole moments of increasing rank. ${ }^{6,33}$ Nuclear centers are typically used as convenient origins to locate 'atomic multipoles', as is 
the case in the "distributed multipole analysis" (DMA), ${ }^{34,35}$ "Atoms in Molecules" (AIM), ${ }^{8}$ and AMOEBA, ${ }^{10}$ to improve convergence of the multipole expansion as opposed to using a single molecular origin. As the rank of these terms increases from dipole to quadrupole to octupole and beyond, the contribution that they make to the electric field in regions beyond the extent of the original charge distribution decays increasingly rapidly with distance. It is therefore often possible to truncate the series expansion at the atomic quadrupole moment ${ }^{36,37}$ while maintaining accuracy of the electrostatic potential outside the molecular surface. This is especially true if the terms of the expansion are fitted to the electrostatic potential rather than derived directly from the electron density. ${ }^{25,38}$

For a discrete distribution of $n$ charges a multipole expansion truncated at the quadrupole moment can be expressed using spherical harmonics as:

$$
\begin{aligned}
Q_{00} & =\sum_{i=1}^{n} q_{i} & Q_{11 s} & =\sum_{i=1}^{n} q_{i} r_{y, i} \\
Q_{10} & =\sum_{i=1}^{n} q_{i} r_{z, i} & Q_{20} & =\sum_{i=1}^{n} \frac{1}{2} q_{i}\left(3 r_{z, i}^{2}-r^{2}\right) \\
Q_{11 c} & =\sum_{i=1}^{n} q_{i} r_{x, i} & Q_{22 c} & =\sum_{i=1}^{n} \sqrt{\frac{3}{4}} q_{i}\left(r_{x, i}^{2}-r_{y, i}^{2}\right)
\end{aligned}
$$

where $q_{i}$ is point charge $i, r_{x, i}$ is the $x$-coordinate of $i$ and $Q_{l m}$ is the total atomic multipole moment of rank $(l, m) .{ }^{6}$ For a continuous charge density an analogous volume integral replaces the summation.

DCM is based on the fact that the converse is also true, i.e. any truncated multipole expansion, even one derived from a continuous charge density, can be represented by a suitable arrangement of discrete point charges. ${ }^{26,39}$ An illustrative but general example is an octahedral charge arrangement, where the magnitude of the charge at each vertex of the octahedron is analytically determined to exactly reproduce all multipole moments up to 
quadrupole according to:

$$
\begin{array}{ccrl}
q_{\left(d_{\mathrm{q}}, 0,0\right)}=\frac{Q_{00}}{6}+\frac{Q_{11 c}}{2 d_{\mathrm{q}}}-\frac{Q_{20}}{6 d_{\mathrm{q}}^{2}}+\frac{Q_{22 c}}{2 \sqrt{3} d_{\mathrm{q}}^{2}} & q_{\left(0,-d_{\mathrm{q}}, 0\right)}=\frac{Q_{00}}{6}-\frac{Q_{11 s}}{2 d_{\mathrm{q}}}-\frac{Q_{20}}{6 d_{\mathrm{q}}^{2}}-\frac{Q_{22 c}}{2 \sqrt{3} d_{\mathrm{q}}^{2}} \\
q_{\left(-d_{\mathrm{q}}, 0,0\right)}=\frac{Q_{00}}{6}-\frac{Q_{11 c}}{2 d_{\mathrm{q}}}-\frac{Q_{20}}{6 d_{\mathrm{q}}^{2}}+\frac{Q_{22 c}}{2 \sqrt{3} d_{\mathrm{q}}^{2}} & q_{\left(0,0, d_{\mathrm{q}}\right)}=\frac{Q_{00}}{6}+\frac{Q_{10}}{2 d_{\mathrm{q}}}+\frac{Q_{20}}{3 d_{\mathrm{q}}^{2}} \\
q_{\left(0, d_{\mathrm{q}}, 0\right)}=\frac{Q_{00}}{6}+\frac{Q_{11 s}}{2 d_{\mathrm{q}}}-\frac{Q_{20}}{6 d_{\mathrm{q}}^{2}}-\frac{Q_{22 c}}{2 \sqrt{3} d_{\mathrm{q}}^{2}} & q_{\left(0,0,-d_{\mathrm{q}}\right)}=\frac{Q_{00}}{6}-\frac{Q_{10}}{2 d_{\mathrm{q}}}+\frac{Q_{20}}{3 d_{\mathrm{q}}^{2}}
\end{array}
$$

Here, $d_{\mathrm{q}}$ is the fixed distance of the charges from the nuclear coordinate of an atom. Note that the $Q_{21 c}, Q_{21 s}$ and $Q_{22 s}$ quadrupole moment components vanish if the correct local axis system for an atom is chosen. ${ }^{26}$

It is therefore possible to replace all 6 nonzero multipole moments by 6 point charges. The total multipole moments of the charge distribution will exactly match the multipole expansion up to truncation rank, so the two will differ only by their higher order terms, i.e. octupole and beyond. These terms can either be kept small by reducing the distance $d_{\mathrm{q}}$, or deliberately enhanced to potentially provide accuracy beyond the truncated multipole expansion by fixing $d_{\mathrm{q}}$ to reproduce some of the higher order multipole moments of the reference atom. The main advantage of such an approach is a considerable reduction in complexity of the terms, as shown explicitly for the quadrupole-quadrupole interaction in section 1 of the SI. Thus, in DCM no fitting is required. Rather, the multipole moments are converted into a distributed charge arrangement based on analytical formulae.

For both multipolar and DCM approaches torques are generated by the off-centered charges or multipole moments that need to be distributed across the surrounding nuclei. As is generally the case in multipolar force fields, the torques of DCM models are applied to the nuclei that define the local (atomic) axis system of each charge (Figure 1), as described elsewhere. ${ }^{26}$ 

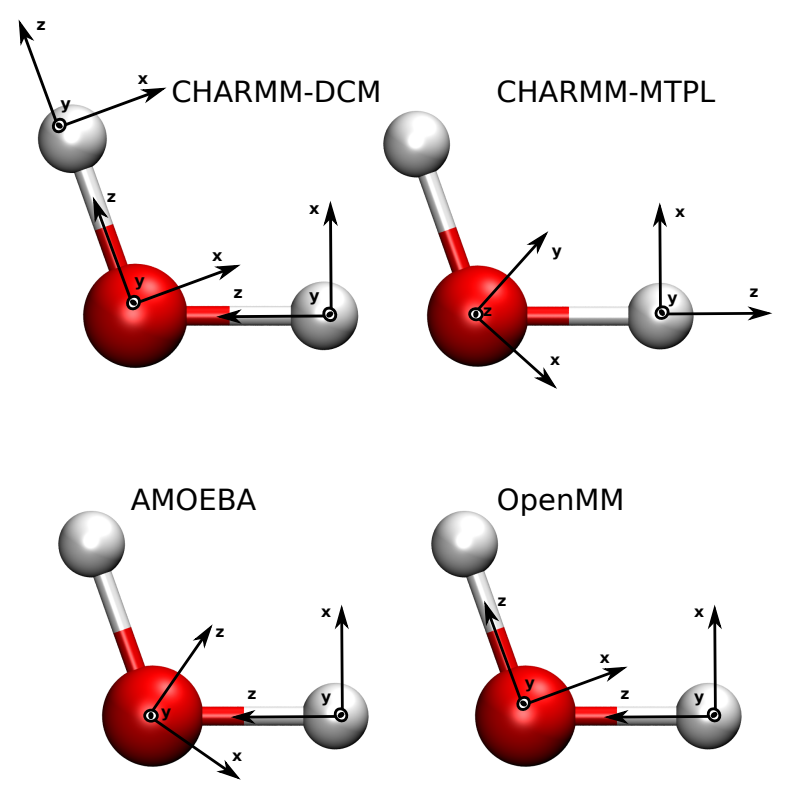

Figure 1: Local axis systems required to convert between the various electrostatic models presented in the text and to implement them into CHARMM and OpenMM. H-atom axes are equivalent except for the case of DCM, where one H-atom shares the same local axis system as its bonded O-atom neighbor.

\section{$2.2 \mathrm{MDCM}$}

The fixed charge arrangements of DCM can be further optimized by releasing constraints on charge positions to exploit spatial degrees of freedom, creating minimal distributed charge models. ${ }^{27}$ For this, MDCM employs machine-learning to determine charge positions and magnitudes such that a predefined accuracy in the MEP is attained using a minimal number of off-centered charges. Differential Evolution ${ }^{40}$ was found to be effective in this context. ${ }^{27}$ Unlike DCMs, MDCMs are not constrained to maintain the same atomic multipole moments as a multipolar reference model, but are fitted directly to describe the MEP around a molecule. Increasing numbers of charges can be added until an MDCM representation reaches a required level of accuracy, with the possibility to even improve beyond what is possible with a multipolar model truncated at quadrupole. ${ }^{27}$ After fitting, each MDCM charge is assigned to a nucleus and the MDCM arrangements are implemented in MD simulations using the same framework (local axis systems, electrostatic cut-offs etc.) as a DCM model. 


\subsection{Polarization}

For improved accuracy and physical rigour and realism, polarization interactions were also included. This makes (M)DCM models viable for emulating the iAMOEBA water model for which polarizable sites were assigned to the atomic nuclei. Here, the 'direct' (nonself-consistent) approach was employed ${ }^{41}$ which allows direct comparison with the original iAMOEBA model. ${ }^{32}$ In this approach, isotropic polarizabilities at nuclear sites are used to add induced dipoles to atoms as a function of the electric field generated by static multipole moments of surrounding atoms only (the field generated by other induced dipoles is ignored). For (M)DCM, this means that the electric field is generated by the point charges of surrounding atoms only. The total polarization energy is therefore:

$$
\begin{array}{r}
V_{\mathrm{pol}}=\sum_{i=1}^{N} \alpha_{i} \mathbf{E}\left(\mathbf{r}_{i}\right)^{2} \\
\mathbf{E}\left(\mathbf{r}_{i}\right)=\sum_{j=1}^{N b_{i}} \sum_{l=1}^{N q_{j}} \frac{\lambda_{i j} q_{l, j} \hat{\mathbf{r}}_{i l}}{R_{i l}^{2}}
\end{array}
$$

where the polarization energy $V_{\text {pol }}$ is determined by a sum over all $N$ atoms of their scalar (isotropic) polarizabilities $\alpha_{i}$ multiplied by the square of the electric field $\mathbf{E}$ at the atom's nuclear position $\mathbf{r}_{i}$. The electric field at the nuclear coordinate of atom $i$ is evaluated by summing over each DCM charge $q_{l}$ of the $N q$ DCM charges belonging to atom $j$, for each atom in the list of $N b$ nonbonded partners of atom $i$ within simulation cut-offs. $\hat{\mathbf{r}}_{i l}$ is a unit vector in the direction of charge $l$ from polarizable center $i, R_{i l}$ is the distance from the

nucleus of atom $i$ to charge $l$. The damping function $\lambda_{i j}$ used in AMOEBA $^{9}$ is also adopted here, with functional form:

$$
\lambda_{i j}=1-\exp \left(-a\left(\frac{R_{i l}}{\left(\alpha_{i} \alpha_{j}\right)^{1 / 6}}\right)^{3}\right)
$$


where $a=0.23616 \AA^{-1} \cdot{ }^{32}$ Although the purpose of the damping function in the original AMOEBA force field was to prevent artifacts at close range (the so-called 'polarization catastrophe'), in the non-iterative case these artifacts should not exist, so $\lambda$ should be interpreted as a fitted short-range correction to the polarization energy.

\subsection{Water Models}

TIP $n \mathbf{P}$ : The parameters for these models are summarized in Table 1 . In all TIP $n \mathrm{P}$ models, the $\mathrm{OH}$ bond length, $r_{\mathrm{OH}}$, and $\mathrm{HOH}$ bond angle, $\angle \mathrm{HOH}$, are the gas-phase experimental values, i.e., $0.9572 \AA$ and $104.52^{\circ}$, respectively. There is no charge at the $\mathrm{O}$ center in both the TIP4P and TIP5P models. The potential energy of the TIP4P and TIP5P models between two water molecules, $a$ and $b$, is given by Eq. 6, where $i$ and $j$ are the charged sites on molecules $a$ and $b$, respectively, and $r_{\mathrm{O}_{\mathrm{a}} \mathrm{O}_{\mathrm{b}}}$ is the oxygen-oxygen distance.

$$
E_{\mathrm{ab}}=4 \epsilon_{\mathrm{OO}}\left[\left(\frac{\sigma_{\mathrm{OO}}}{r_{\mathrm{O}_{\mathrm{a}} \mathrm{b}}}\right)^{12}-\left(\frac{\sigma_{\mathrm{OO}}}{r_{\mathrm{O}_{\mathrm{a}} \mathrm{b}}}\right)^{6}\right]+\sum_{i j} \frac{q_{i} q_{j}}{r_{i j}}
$$

Eq. 6 is equally applicable for TIP3P water when including an additional L-J interaction term on hydrogen sites. As the DCM approach uses off-centered charges to describe multipole moments, no modification is necessary to implement the TIP $n \mathrm{P}$ models, which are equivalent to MDCM distributions with 1 or 2 charges per atom. In some respects the $\mathrm{TIP} n \mathrm{P}$ models can be viewed as MDCMs with hand-fitted charge positions and magnitudes, as displayed in Figure 2.

iAMOEBA: Inexpensive AMOEBA ${ }^{32}$ was originally conceived as a computationally efficient (fewer parameters and non-iterative polarization) and robust alternative to the existing AMOEBA water model. The requisite Halgren buffered 14-7 potential $^{42}$ and anharmonic

bonded terms of the iAMOEBA model are available in OpenMM, ${ }^{29}$ which also contains basic "dummy atom" functionality for sites with zero mass that can be used to run simulations 
TIP3P
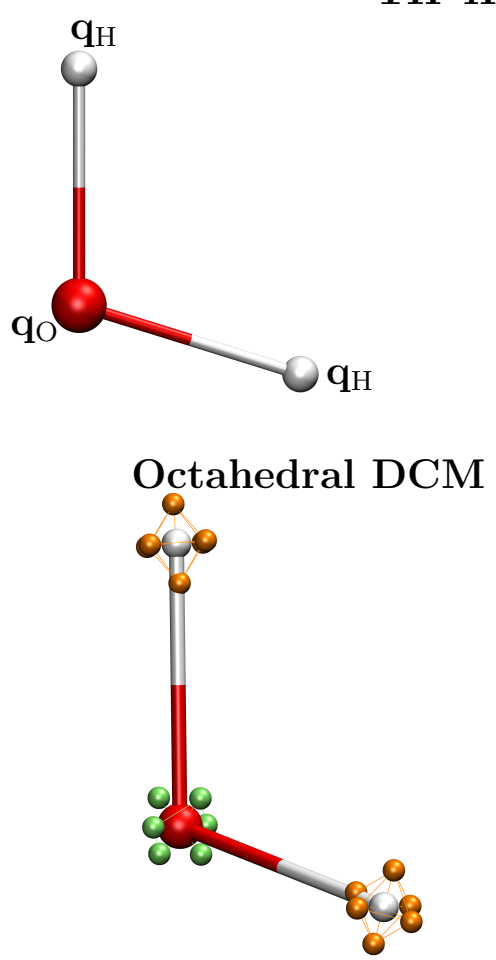

TIP4P

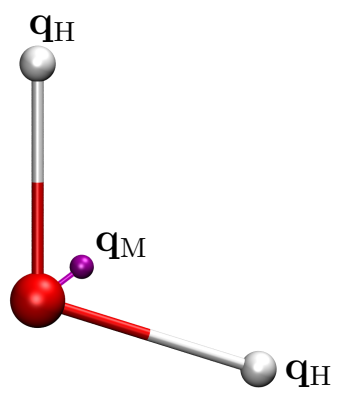

10-charge MDCM

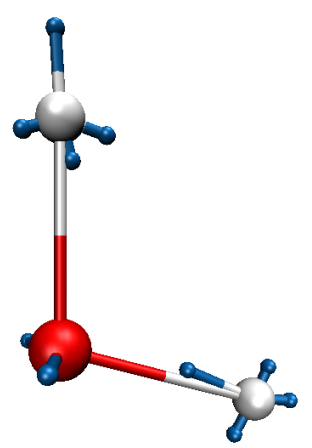

Figure 2: Charge locations in $\operatorname{TIP} n \mathrm{P}(n=3,4,5)$ water models (top), in an octahedral distributed charge model (DCM, bottom-left) and a 10-charge minimal distributed charge model (MDCM, bottom-right). In all TIP $n \mathrm{P}$ models positive charges are located at the $\mathrm{H}$ centers. The corresponding negative charges are located either at the $\mathrm{O}$ atom $\left(q_{\mathrm{O}}\right.$ in TIP3P), along an axis connecting $\mathrm{O}$ and the center of mass of $\mathrm{H}_{2} \mathrm{O}\left(q_{\mathrm{M}}\right.$ in TIP4P), or at two points approximating the $\mathrm{O}$ lone pairs $\left(q_{\mathrm{L}}\right.$ in TIP5P). In the octahedral DCM model there are 6 charge sites for the $\mathrm{O}$ atom and for each $\mathrm{H}$ atom, describing the multipole moments of the iAMOEBA model. In the MDCM model there are 4 charge sites per $\mathrm{H}$ atom and 2 charge sites for $\mathrm{O}$.

with (M)DCMs. iAMOEBA in OpenMM thus provides a suitable test case to compare performance of explicit atomic multipole moments versus distributed charges in polarizable, condensed phase simulations. It is also possible to demonstrate the ease with which (M)DCMs can be made available in existing MD software packages without the need to change the source code. Such an "emulation" should offer advantages in both computational efficiency and ease of implementation with respect to adding explicit multipolar interaction terms to software that does not already have this functionality. 
Table 1: Parameters for TIP $n \mathrm{P}$ and iAMOEBA potential functions.

\begin{tabular}{|c|c|c|c|c|}
\hline & TIP3P & TIP4P & TIP5P & $\begin{array}{c}\text { iAMOEBA } \\
(\mathrm{M}) \mathrm{DCM}\end{array}$ \\
\hline \multicolumn{5}{|l|}{ Geometry } \\
\hline$\angle \mathrm{HOH}^{\circ}$ & 104.52 & 104.52 & 104.52 & 106.48 \\
\hline$\angle \mathrm{LOL}^{\circ}$ & & & 109.47 & \\
\hline $\mathrm{r}_{\mathrm{OH}} / \AA$ & 0.9572 & 0.9572 & 0.9572 & 0.9584 \\
\hline $\mathrm{r}_{\mathrm{OL}} / \AA$ & & & 0.70 & \\
\hline $\mathrm{r}_{\mathrm{OM}} / \AA$ & & 0.15 & & \\
\hline \multicolumn{5}{|l|}{ Charges } \\
\hline $\mathbf{q}_{\mathrm{H}} / e$ & 0.417 & 0.52 & 0.241 & $\dagger$ \\
\hline $\mathbf{q}_{\mathrm{o}} / e$ & -0.834 & & & $\ddagger$ \\
\hline $\mathbf{q}_{\mathrm{L}} / e$ & & & -0.241 & \\
\hline $\mathbf{q}_{\mathrm{M}} / e$ & & -1.04 & & \\
\hline \multicolumn{5}{|l|}{ L-J parameters } \\
\hline$\epsilon_{\mathrm{OO}} / \mathrm{kcal} / \mathrm{mol}$ & -0.1521 & -0.155 & -0.16 & $0.19682^{a}$ \\
\hline$\epsilon_{\mathrm{HH}} / \mathrm{kcal} / \mathrm{mol}$ & -0.0460 & & & \\
\hline$\sigma_{\mathrm{OO}} / \AA$ & 3.15061 & 3.15365 & 3.12 & $3.6453^{a}$ \\
\hline$\sigma_{\mathrm{HH}} / \AA$ & 0.4 & & & \\
\hline \multicolumn{5}{|l|}{ Polarizabilities } \\
\hline$\alpha_{\mathrm{O}} / \AA^{3}$ & & & & 0.80636 \\
\hline$\alpha_{\mathrm{H}} / \AA^{3}$ & & & & 0.50484 \\
\hline$a / \AA^{-1}$ & & & & $0.23616^{b}$ \\
\hline
\end{tabular}

$\dagger(\mathrm{M}) \mathrm{DCM}$ charge sites for $\mathrm{H}$ atoms, and $\ddagger(\mathrm{M}) \mathrm{DCM}$ charge sites for $\mathrm{O}$ atoms - refer to section 2 of the SI.

${ }^{a}$ iAMOEBA L-J parameters for use with a Halgren 14-7 potential $^{42}$

${ }^{b}$ polarization damping factor

\section{Computational Details}

\subsection{DCM Representations}

TIP $n \mathrm{P}$ models were implemented for use with the DCM module in CHARMM by describing the positions of any off-centered charges in the standard DCM local axis system. ${ }^{26}$ For multipolar models, in-house scripts were used to convert from the original local axis system, as defined here by the AMOEBA force field for the iAMOEBA model and the multipole 
module of the CHARMM force field for the multipolar PhF model, to the final DCM axis system. Also, the necessary diagonalization of the Cartesian quadrupole matrix to obtain a minimal number of non-zero quadrupole components and to calculate the corresponding charge magnitudes of the DCM arrangement were computed using additional scripts that have been made available online. ${ }^{43}$ An octahedral charge arrangement was used to describe the multipole expansion for all DCM models, as described previously. ${ }^{26}$ For the polarization term the standard iAMOEBA damping parameters were used (Table 1). Polarizabilities were corrected as described in section 3.3 below, to maintain the original AMOEBA multipolar force field term and avoid refitting polarization damping parameters. A sample input file is provided in section 3 of the SI.

\subsection{MDCM fitting}

The Differential Evolution (DE) fitting code was implemented into the "Fitting Wizard" (FW) tool previously developed to fit multipole moments and L-J parameters to bulk properties for multipolar force fields. ${ }^{44}$ Charge positions were constrained using hard constraints during DE fitting (candidate solutions that violated constraints were labeled "unfeasible") so that all charges remained within one third of the van der Waals radius of an atom. Atomic multipoles up to rank $l=5$ were fitted to the MEP across a grid using a least squares fit as before,${ }^{27}$ where the grid used here is generated by the target multipolar model of interest rather than quantum chemical reference data. Specifically, the iAMOEBA and PhF multipole moments were used to evaluate the MEP at each grid point of a rectangular grid with spacing $0.1 \AA$ and dimensions $11.4 \times 11.0 \times 10.1 \AA\left(\mathrm{H}_{2} \mathrm{O}\right)$ or $14.4 \times 10.2 \times 15.2 \AA(\mathrm{PhF})$ centered on the molecule.

Grid points between the 0.001 a.u. and 0.0003 a.u. isodensity surfaces were used for fitting, as points outside the outer 0.0003 a.u. surface with lower electron density were found to be 
far enough away to be generally well described and have only a small impact on fitting quality. Points outside the outer surface that were excluded for fitting were, however, included to validate the performance of the model for the long-range part of the interaction. Grid points within the 0.001 a.u. isodensity surface were discarded for both fitting and subsequent evaluation of the fit.

Atomic charge models with up to 4 charges per atom were fitted to the ESP generated by the $l=5$ atomic multipoles for both molecules and used to generate initial populations for subsequent DE fitting of the larger systems, as described elsewhere. ${ }^{27} \mathrm{MDCM}$ was algorithmically improved by introducing an intermediate fragmentation strategy to increase computational efficiency for larger molecules. After fitting atomic charge models to the ESP of the atomic multipoles, the PhF molecule was thus divided into fragments (here into 2 arbitrary fragments of roughly equal size). Next, each fragment was fitted separately to a reference fragment ESP that does not include the ESP contribution of the multipoles of the other fragment(s). This is achieved using the high-rank $(l=5)$ fitted atomic multipoles already obtained to fit the atomic charge models:

$$
V_{\text {ref }}^{\mathrm{frag}}(\mathbf{r})=V_{\text {ref }}^{\mathrm{mol}}(\mathbf{r})-\sum_{i=1}^{N_{\text {frag, fix }}} \sum_{j=1}^{N_{\text {atom }, i}} V_{i, j}^{\mathrm{mtp}}(\mathbf{r})
$$

where the fragment reference ESP $V_{\text {ref }}^{\text {frag }}$ at point $\mathbf{r}$ is equal to the original reference MEP, $V_{\text {ref }}^{\text {mol }}(\mathbf{r})$, minus the ESP $V_{i, j}^{\mathrm{mtp}}(\mathbf{r})$ from the fitted multipoles of all $j$ atoms of all $N_{\text {frag,fix }}$ fragments that are not included in the current fragment fit. As each fragment contains fewer charges than the full molecule, and all fragments can be fitted independently a considerable speedup of the fitting process is possible and the approach scales favourably for larger systems. For $\mathrm{H}_{2} \mathrm{O}$ no fragmentation was necessary and molecular MDCMs were fitted directly to the iAMOEBA reference MEP. Scripts and code required for this workflow have been made available online. ${ }^{43}$ 
Fragments $(\mathrm{PhF})$ or molecules $\left(\mathrm{H}_{2} \mathrm{O}\right)$ are fitted with increasing numbers of charges until a predefined/desired accuracy has been obtained. Here, an average of between 1 and 3 charges per atom $(\mathrm{PhF})$ or 2 and 3.3 charges $\left(\mathrm{H}_{2} \mathrm{O}\right)$, respectively, was trialled during fitting and charges were free to move. The initial fragment or molecular DE population is assembled from combinations of atomic charge models that yield the lowest RMSE, typically by assigning more charges to atoms with more challenging ESP distributions. Hence, the number of charges for each atom within the fragment or molecule may differ in the initial population, and may also change during fitting, while the total number of charges for the fragment or molecule remains fixed. For each given total number of charges ten independent models for each $\mathrm{PhF}$ fragment or $\mathrm{H}_{2} \mathrm{O}$ molecular model were fitted. After fitting fragment models for $\mathrm{PhF}$, those with lowest RMSE across the ESP grid were combined to build molecular charge models with the total number of charges also corresponding to between 1 and 3 charges per atom (i.e. between 12 and 36 charges for the full $\mathrm{PhF}$ molecule) on average. Each molecular $\mathrm{PhF}$ model was subjected to a final DE refinement step.

A further improvement to the original approach ${ }^{27}$ was to introduce additional constraints on charge magnitudes. Constraints are important both to maintain stability of MD simulations and to maintain accuracy of subsequent electrostatic interaction energy calculations. Simulation stability is maintained by constraining charge positions to remain within $r_{\text {atom }} / 3$, one third of the van der Waals radius of the atom. If charges are placed too far from nuclear positions they are able to approach one another during MD simulations, overcoming repulsive barriers and causing simulations to collapse due to numerical instability. To improve the accuracy of electrostatic interaction energies, hard constraints of maximally $1 e$ for each point charge were applied to all charge magnitudes. The grounds for constraining charge magnitudes is based on analysis of the error in the interaction energy using MDCMs (described in section 4 of the SI), as larger charge magnitudes often reduce the error in the MEP 
at the expense of increasing the error in the electrostatic interaction energy in subsequent simulations through error multiplication.

\section{$3.3 \quad(\mathrm{M}) \mathrm{DCM}$ in OpenMM}

For iAMOEBA simulations, a single simulation engine (OpenMM 7.1.0 ${ }^{45}$ ) was used for multipolar and (M)DCM models to keep all simulation details and force field terms unchanged apart from the modified electrostatics. As OpenMM lacks native DCM support, the existing "dummy atom" functionality was exploited to run (M)DCM simulations, highlighting the possibility to run (M)DCM simulations in simulation packages that support dummy atoms or equivalent features. (M)DCM charges were placed relative to atoms by converting from local DCM axes to those defined in OpenMM for dummy atoms (Figure 1).

For consistency, the polarization term in OpenMM had to be adapted for use with distributed charges. The polarization energy damping term implemented in OpenMM assumes that charged and polarizable sites will coincide, as the distance $R_{i l}$ of the damping term in Eq. 4 is evaluated between nuclear sites. This is not the case in (M)DCM, where charge sites are shifted from nuclear positions. If every (M)DCM charge site were assigned the polarizability of the parent atom, the total polarization energy would be significantly overestimated, in accordance with Eq. 3. Hence, $\alpha_{i}^{\prime}=\alpha_{i} \cdot 10^{-4}$ was used for charge positions and the damping factor $\lambda_{i j}$ was changed to

$$
\lambda_{i j}=1-\exp \left(-\frac{a^{\prime}}{100}\left(\frac{r_{i l}}{\left(\alpha_{i}^{\prime} \alpha_{j}^{\prime}\right)^{1 / 6}}\right)^{3}\right)
$$

with $a^{\prime}=0.0023616 \AA^{-1}$ and $\alpha_{i}^{\prime}=\alpha_{i}$ for the nuclei. As all nuclei carry zero charge in (M)DCMs the nuclear-nuclear interactions yield zero polarization energy, in accordance with

Eq. 4. As all charges carry polarizabilities scaled by a factor $10^{-4}$, polarization energies be- 
tween (M)DCM charges are negligible which is consistent with Eq. 3. For charge-nuclear site interactions, the nuclear site carries the standard polarizability, so Eq. 3 is unchanged, and the charge site polarizability is scaled by $10^{-4}$, which is counteracted by the factor $10^{-2}$ applied to the damping parameter $a$ in Eq. 5, recovering the polarization and damping of the multipolar force field without refitting any parameters. Note that the small remaining difference in the $R_{i l}$ term from using shifted charge sites was found to not significantly affect the results, but should be considered a potential source from which slight differences can arise.

Finally, fitted MDCMs were converted to the standard local axis systems used for dummy atoms in OpenMM. A sample parameter file is provided in section 5 of the SI.

\subsection{Simulations and Property Computation}

Aside from iAMOEBA simulations, which were run with OpenMM as described below, remaining MD simulations were run with $\mathrm{CHARMM}^{28}$ version $45 \mathrm{a} 2$ which includes provisions for DCM. ${ }^{26}$ A 1 fs time step was used with SHAKE ${ }^{46}$ to constrain angles and bonds involving hydrogen atoms in an isothermal-isobaric (constant NPT) ensemble using a pressure bath at 1 atm coupled to a Nosé-Hoover temperature bath. ${ }^{47-51}$ The simulation system was a cubic box with 500 water molecules employing periodic boundary conditions. For every value of $T$, a simulation of at least 3 nanoseconds (ns) was performed. For TIP5P water and temperatures below freezing point $(273 \mathrm{~K})$ the simulations were extended by an additional $3 \mathrm{~ns}$ for improved estimates of thermodynamic properties using fluctuation formulae (see below). This strategy has been suggested previously to obtain converged results for modeling bulk water at low temperatures. ${ }^{52-55}$ All simulations were performed with SHIFT and SWITCH cutoff functions ${ }^{56}$ for non-bonded electrostatics and van der Waals interactions, respectively. The switching-function parameters are $R_{\text {on }}$ and $R_{\text {off }}$ with values 10.0 and 12.0 $\AA$, respectively, for non-bonded van der Waals interactions. A $12.0 \AA$ cutoff was applied 
for the shifted non-bonded electrostatics. For atoms with off-centered charges, distances for the shifting function were measured between the off-centered charge sites. The TIP4P and TIP5P simulations in particular provide a stringent validation for the implementation of cut-offs, torques, and integration with barostats of (M)DCM into CHARMM by comparing with results of the same simulations from those using standard routines. ${ }^{57} \mathrm{PME}$ for DCM has not yet been implemented in CHARMM.

OpenMM simulations of a cubic box with 500 water molecules were run without SHAKE constraints for compatibility with iAMOEBA, and with a 0.5 fs time step. A Monte Carlo barostat maintained simulation pressure at $1 \mathrm{~atm}$, after $150 \mathrm{ps}$ equilibration simulations were run for $10 \mathrm{~ns}$ at each $T$ used for the CHARMM simulations to facilitate direct comparison between CHARMM and OpenMM data. Particle Mesh Ewald (PME) was used with a realspace cutoff of $7.0 \AA$ and a van der Waals cutoff of $9.0 \AA$.

Solvation Free Energies, $\Delta G$, for $\mathrm{PhF}$ were calculated using a thermodynamic integration (TI) procedure described elsewhere, ${ }^{58}$ with slow-growth thermodynamic integration ${ }^{59,60}$ for LJ interactions with discrete windows of $\lambda$ from 0 to $1, \Delta \lambda=0.1$ and $\Delta t=1 \mathrm{fs}$, averaging over 8 separate forwards and backwards trajectories. $\lambda$ windows with an energy variance of $>0.5 \mathrm{kcal} / \mathrm{mol}$ were halved and simulations were repeated for the smaller window size. Although the DCM module in CHARMM is integrated with existing slow-growth algorithms, for direct comparison with multipole results the electrostatic contribution to the solvation free energy was evaluated by performing simulations with solute electrostatics scaled by $\lambda_{\text {mid }}$, where $\lambda_{\text {mid }}$ is the $\lambda$ value corresponding to the midpoint of the $\lambda$ window, again for discrete windows of $\lambda$ from 0 to 1 , with fixed $\Delta \lambda=0.05$ and $\Delta t=1 \mathrm{fs}$. A post-processing step then recalculated energies using the unscaled Hamiltonian for each time step of the simulation with scaled solute electrostatics. ${ }^{58}$ The simulations for each value of $\lambda$ for each of the 8 runs were equilibrated for $50 \mathrm{ps}$, and then sampled for another $100 \mathrm{ps}$, i.e., cumulatively $8 \times 150$ 
ps for each TI window.

Bulk-density $\rho$ was computed from the ratio between total mass, $M$, and the time-averaged volume of the simulation box, $\langle V\rangle$ according to $\rho=\frac{M}{\langle V\rangle}$.

Self-diffusion coefficient $D$ was computed from the mean squared displacement (MSD) of all oxygen atoms using the Einstein relation

$$
D=\lim _{t \rightarrow \infty} \frac{1}{6 t}<|r(t)-r(0)|^{2}>
$$

where $r(t)$ is the position of the oxygen atom of a water molecule at time $t$, and averaged over all water molecules. ${ }^{61}$ OpenMM trajectories were analyzed in CHARMM and $D$ was computed in the same way.

Enthalpy of vaporization $\Delta H_{\text {vap }}$ can be obtained from

$$
\Delta H_{\text {vap }}=<E_{\text {gas }}>-<E_{\text {liq }}>/ N+R T,
$$

where $E_{\text {liq }}$ is the potential energy of the liquid containing $N$ molecules and $R$ is the ideal gas constant. ${ }^{52,53,62}$

Heat capacity $C_{p}$, isothermal compressibility $\kappa$, coefficient of thermal expansion $\alpha$ can be calculated from standard fluctuation formulae (Eqs. 11 to 13). ${ }^{52,53,62}$

$$
C_{p}=\left(\frac{\partial H}{\partial T}\right)_{N, P}=\frac{1}{N k_{B} T^{2}}\left(<H^{2}>-<H>^{2}\right)+3 R
$$




$$
\begin{gathered}
\kappa=-\frac{1}{V}\left(\frac{\partial V}{\partial P}\right)_{N, T}=\frac{1}{k_{B} T<V>}\left(<V^{2}>-<V>^{2}\right) \\
\alpha=\frac{1}{V}\left(\frac{\partial V}{\partial T}\right)_{N, P}=\frac{1}{k_{B} T^{2}<V>}(<V H>-<V><H>)
\end{gathered}
$$

Here, $C_{p}$ and $\alpha$ were computed using the central difference formula for estimating derivatives, except at extremes where right and left differences were used, ${ }^{63}$

$$
C_{p} \approx \frac{<H_{2}>-<H_{1}>}{T_{2}-T_{1}} \text {, and } \quad \alpha \approx \frac{\ln <\rho_{2}>-\ln <\rho_{1}>}{T_{2}-T_{1}}
$$

and $\kappa$ values were calculated from the fluctuations (Eq. 12).

\section{Results and Discussion}

This section is structured as follows. First, the performance of the (M)DCM iAMOEBA model is evaluated for single-point energy calculations of water clusters, in order to validate the (M)DCM models against reference data from the original multipolar iAMOEBA implementation. Implementations for the TIP $n \mathrm{P}$ models are straightforward as the original charge coordinates are used directly, rather than replaced by an (M)DCM, and are hence not shown here. Then the performance of all models for different bulk properties between $T=235.5 \mathrm{~K}$ and $T=350 \mathrm{~K}$ is assessed to explore the performance of the DCM framework in describing models of increasing complexity under realistic simulation conditions. For iAMOEBA the impact on simulations of replacing the multipole term with distributed charges is the main focus. For TIP $n$ P the focus is compatibility of the DCM dynamics framework with existing, simpler charge models with and without off-centered charges. Finally, the methods developed are further used for MD and free energy simulations of hydrated $\mathrm{PhF}$. 


\subsection{MDCM Fitting for $\mathrm{H}_{2} \mathrm{O}$}

Models with increasing numbers of charges ranging from 6 to 10 per molecule were fitted to the MEP, with 10 independent models fitted for each number of charges. The models that performed best as quantified by mean absolute error in the MEP were then used in dimer energy calculations (next section). As no refitting of other force field parameters was desirable a tight agreement of $\sim 0.2 \mathrm{kcal} / \mathrm{mol}(1 \mathrm{~kJ} / \mathrm{mol})$ in dimer energies was the threshold to select the MDCM to be used in subsequent calculations. It was found that 10 charges were required to reach this threshold, more than might typically be necessary for such a model. However, this is justified by the challenging nature of the task, and still compares favorably with the 12 non-zero multipole components of the iAMOEBA model and without

the additional complexity that these terms will incur in simulations. In a more typical application an error in dimer electrostatic interaction energies of $1 \mathrm{kcal} / \mathrm{mol}$ with respect to $a b$ initio reference data might be acceptable, requiring fewer charges, as errors are typically compensated by remaining force field terms. The MAE across the grid between the two isodensity surfaces for the selected model was $0.023 \mathrm{kcal} / \mathrm{mol}$ and the maximum absolute error was $2.10 \mathrm{kcal} / \mathrm{mol}$.

The selected model is shown at the bottom-right of Figure 2. No symmetry constraints were applied in the fitting. While such constraints would represent a useful extension for the particular case of systems with symmetry, the asymmetric distribution of MDCMs obtained during fitting still accurately describes the symmetry of the underlying MEP if the fitting criteria are sufficiently tight. This is explicitly discussed for the water cluster energies in section S6 of the SI. There, it is demonstrated that minimal error is incurred by rotating monomers 180 degrees about their $\mathrm{H}-\mathrm{O}-\mathrm{H}$ bisectors, with maximal difference of $\Delta E \leq 0.027$ $\mathrm{kcal} / \mathrm{mol}$ for dimer energies and $\Delta E \leq 0.019 \mathrm{kcal} / \mathrm{mol} / \mathrm{monomer}$ for clusters up to decamer, and mean absolute $\Delta E=0.039 \mathrm{kcal} / \mathrm{mol}$ over all dimers and clusters. Both orientations can 
therefore be used equivalently in simulations without affecting bulk properties, as further demonstrated in sections 4.3 to 4.6 below.
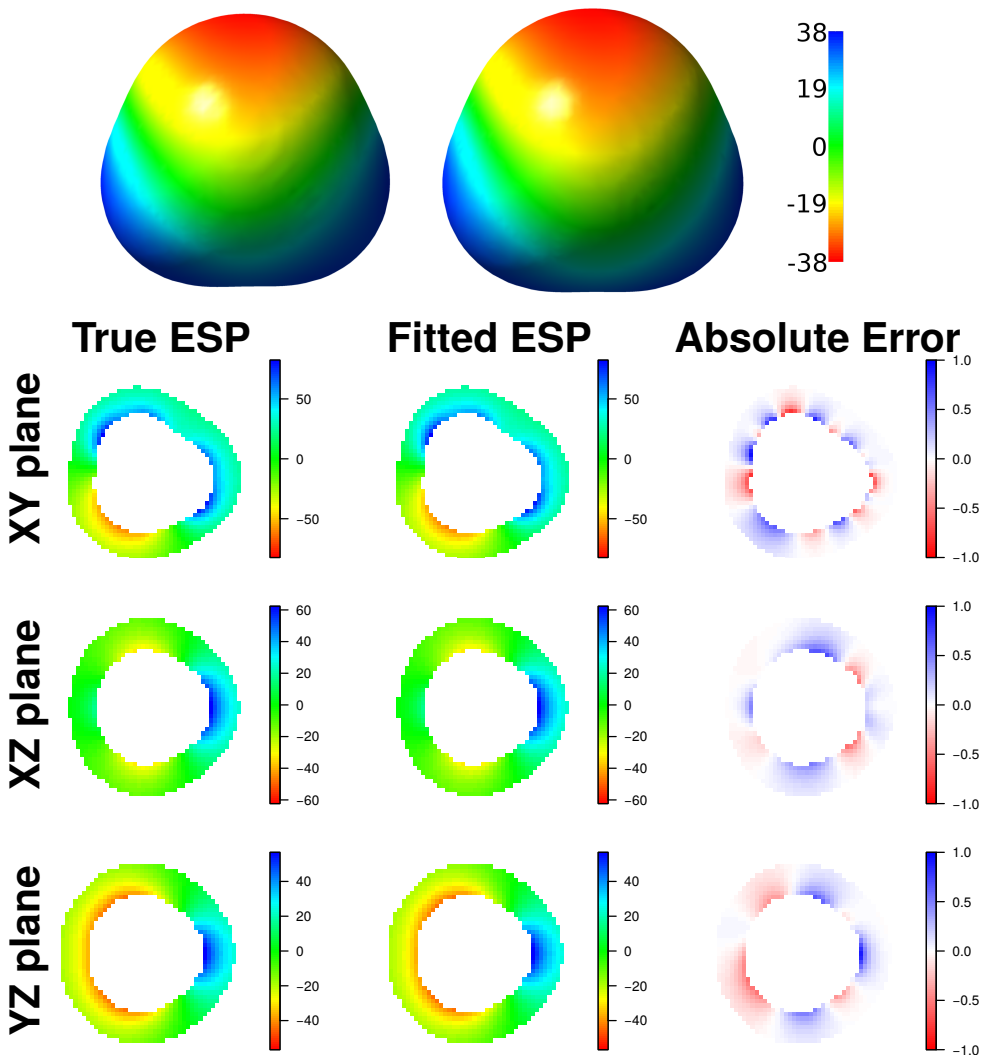

Figure 3: Top: comparison of MEP generated by 10-charge MDCM (left) with MEP generated by iAMOEBA multipole moments (right), mapped onto the molecular 0.001 a.u. isodensity surface. Colors range from -38 (red) to +38 (blue) kcal/mol. Bottom: 2D slices of the MEP in different molecular planes (rows) in the region used for fitting. The "True" ESP refers to the iAMOEBA multipolar reference, the fitted ESP refers to the MDCM 10-charge model. The absolute error is plotted in the right-hand column and ranges from $-1 \mathrm{kcal} / \mathrm{mol}$ (red) to $+1 \mathrm{kcal} / \mathrm{mol}$ (blue).

\section{2 iAMOEBA and its (M)DCM Representation}

The quality of the octahedral DCM and 10-charge MDCM descriptions of the iAMOEBA multipolar electrostatics were examined using a series of water dimers, originally proposed by Tschumper et al. ${ }^{64}$ (Figure 4 ) and a set of larger water clusters up to decamer ${ }^{65}$ to check for error accumulation with cluster size. 
Table 2: Comparison between multipolar and DCM iAMOEBA electrostatic energies, including polarization. In the top part of the table, energies for the 10 dimer structures (see Figure 4) are shown, while in the bottom part energies for various oligomers (see Figure 5) are presented. The mean absolute error for the dataset is within chemical accuracy (MAE $=0.041$ $\mathrm{kcal} / \mathrm{mol}$ )

\begin{tabular}{l|ccc} 
Dimer & iAMOEBA $(\mathrm{kcal} / \mathrm{mol})$ & DCM-iAMOEBA $(\mathrm{kcal} / \mathrm{mol})$ & $\Delta E(\mathrm{kcal} / \mathrm{mol})$ \\
\hline 1 & -5.113 & -5.092 & 0.021 \\
2 & -4.505 & -4.532 & -0.027 \\
3 & -4.504 & -4.554 & -0.049 \\
4 & -3.824 & -3.769 & 0.056 \\
5 & -3.269 & -3.217 & 0.053 \\
6 & -2.972 & -2.967 & 0.005 \\
7 & -3.199 & -3.173 & 0.027 \\
8 & -1.572 & -1.579 & -0.007 \\
9 & -3.794 & -3.778 & 0.016 \\
10 & -3.016 & -3.027 & -0.011 \\
\hline Oligomer & iAMOEBA $(\mathrm{kcal} / \mathrm{mol})$ & DCM-iAMOEBA $(\mathrm{kcal} / \mathrm{mol})$ & $\Delta E(\mathrm{kcal} / \mathrm{mol})$ \\
\hline trimer & -13.770 & -13.691 & 0.079 \\
tetramer & -24.529 & -24.495 & 0.034 \\
pentamer & -32.321 & -32.279 & 0.067 \\
hexamer prism & -41.459 & -41.421 & 0.038 \\
heptamer & -51.299 & -51.193 & 0.106 \\
octamer & -64.672 & -64.495 & 0.178 \\
nonamer & -72.896 & -72.989 & -0.092 \\
decamer & -82.858 & -82.772 & 0.086 \\
& & &
\end{tabular}


Table 3: Comparison between multipolar and MDCM iAMOEBA electrostatic energies, including polarization. In the top part of the table, energies for the 10 dimer structures are shown, while in the bottom part energies for various oligomers are presented. The mean absolute error for the dataset is within chemical accuracy (MAE $=0.108 \mathrm{kcal} / \mathrm{mol}$ )

\begin{tabular}{l|ccc} 
Dimer & iAMOEBA $(\mathrm{kcal} / \mathrm{mol})$ & MDCM-iAMOEBA $(\mathrm{kcal} / \mathrm{mol})$ & $\Delta E(\mathrm{kcal} / \mathrm{mol})$ \\
\hline 1 & -5.113 & -5.125 & -0.011 \\
2 & -4.505 & -4.465 & 0.040 \\
3 & -4.504 & -4.471 & 0.033 \\
4 & -3.824 & -3.836 & -0.011 \\
5 & -3.269 & -3.180 & 0.089 \\
6 & -2.972 & -2.863 & 0.109 \\
7 & -3.199 & -3.196 & 0.003 \\
8 & -1.572 & -1.587 & -0.014 \\
9 & -3.794 & -3.770 & 0.024 \\
10 & -3.016 & -3.026 & -0.010 \\
\hline Oligomer & iAMOEBA $(\mathrm{kcal} / \mathrm{mol})$ & MDCM-iAMOEBA $(\mathrm{kcal} / \mathrm{mol})$ & $\Delta E(\mathrm{kcal} / \mathrm{mol})$ \\
\hline trimer & -13.770 & -13.782 & -0.012 \\
tetramer & -24.529 & -24.436 & 0.093 \\
pentamer & -32.321 & -32.266 & 0.055 \\
hexamer prism & -41.459 & -41.348 & 0.111 \\
heptamer & -51.299 & -51.129 & 0.171 \\
octamer & -64.672 & -64.238 & 0.434 \\
nonamer & -72.896 & -72.478 & 0.419 \\
decamer & -82.858 & -82.402 & 0.456 \\
& & &
\end{tabular}




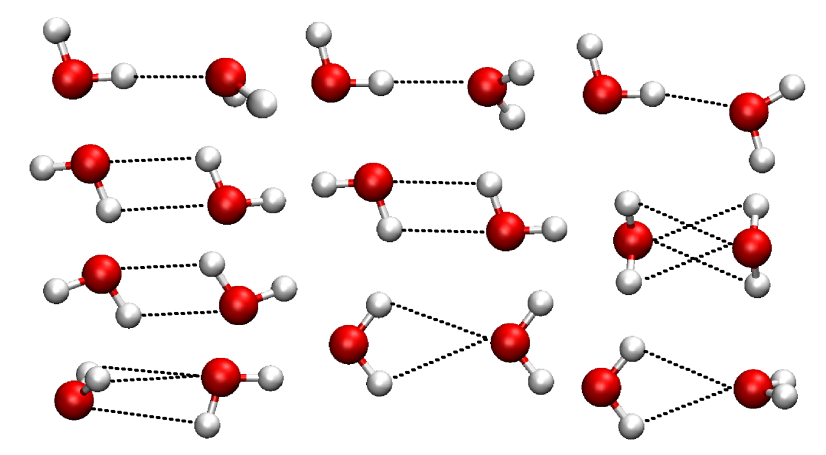

Figure 4: Water dimers from Ref. ${ }^{64}$ used to validate (M)DCM iAMOEBA parameters.

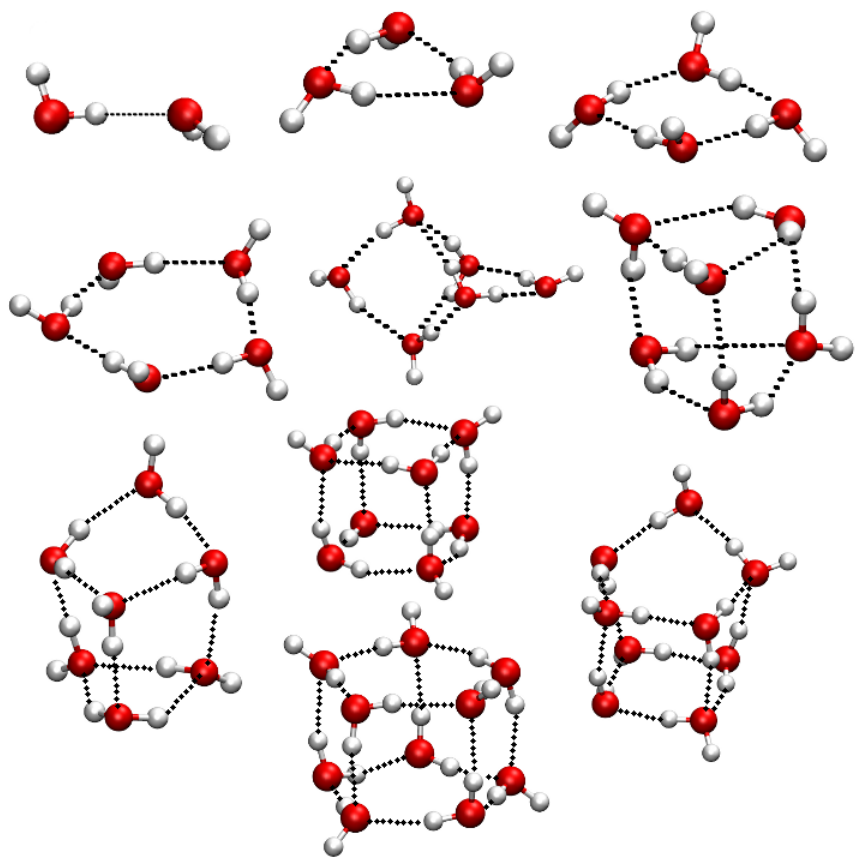

Figure 5: Water clusters used to check for error accumulation in (M)DCM iAMOEBA model.

As seen in Table 2, very close agreement was obtained between the original multipolar iAMOEBA implementation in OpenMM and its DCM representation developed here. The difference in electrostatic interaction energy (including polarization) is of the order of $10^{-2}$ $\mathrm{kcal} / \mathrm{mol}$ for all dimers. Close agreement was also obtained for the water clusters, with error accumulation remaining remarkably small up to decamer, and the largest total error of 0.18 $\mathrm{kcal} / \mathrm{mol}$ still well within chemical accuracy.

Table 3 reveals a similar trend for the fitted MDCM. While dimer errors are slightly larger 
than for the DCM, the largest error of $0.109 \mathrm{kcal} / \mathrm{mol}$ is still very close to the iAMOEBA multipolar energy. For the larger clusters results are again encouraging, although some error accumulation is visible as errors increase to almost $0.5 \mathrm{kcal} / \mathrm{mol}$ for the decamer. It should be emphasized that this remains well within chemical accuracy even for these larger clusters, with a percentage error of $0.5 \%$ for the decamer and MAE of $0.11 \mathrm{kcal} / \mathrm{mol}$ for the whole set of dimers and clusters, despite requiring little more than half the number of charges used in the octahedral DCM.

\section{3 $\quad \mathrm{H}_{2} \mathrm{O}$ Thermodynamic Properties at $298 \mathrm{~K}$ from all Models}

After establishing that both the octahedral DCM and 10-charge MDCM yield accurate interaction energies, the performance of the models for bulk properties in condensed phase MD simulations was assessed. (M)DCM routines and axis systems for models ranging from TIP3P to iAMOEBA and (M)DCM charge representations of iAMOEBA were chosen to demonstrate the versatility of the approach. For multipolar models, simulations are carried out for both the original model and its (M)DCM representation. For TIP $n \mathrm{P}$ models, simulations are carried out using both preexisting code with routines for off-centered charges, and the new DCM implementation in CHARMM.

Atom-atom pair correlation functions (radial distribution functions, RDFs) $g_{\mathrm{OO}}(r), g_{\mathrm{OH}}(r)$ and $g_{\mathrm{HH}}(r)$ characterize the microscopic structure of liquid water. One of the critical tests for water models is accurate reproduction of the experimental (X-ray scattering and neutron diffraction) $\mathrm{OO}, \mathrm{OH}$ and $\mathrm{HH}$ RDFs, although it should be noted that there remains some uncertainty over reference values in the literature. For example, the reported height $(g 1)$ and position $(r 1)$ of the first intermolecular peak in $g_{\mathrm{OO}}(r)$, which is the characteristic feature of liquid water, varies over the range $g 1=2.2-3.0$ and $r 1=2.76-2.82 \AA$ depending on the type of experiment. ${ }^{66-75}$ 
Figure 6 shows $g_{\mathrm{OO}}(r)$ for the various water models along with experimental neutron diffraction data. ${ }^{66}$ A more detailed comparison of $g_{\mathrm{OO}}(r)$ along with $g_{\mathrm{OH}}(r)$ and $g_{\mathrm{HH}}(r)$ is presented in sections $7-9$ of the SI. Of particular interest here is the close agreement firstly between $\mathrm{TIP} n \mathrm{P}$ results and their DCM equivalents. The DCM implementation in CHARMM was conceived to efficiently run dynamics for distributed charge representations of multipolar electrostatic models. Decisions such as how to implement cut-offs and torques, and interactions with barostats may therefore yield different results to widely used dummy-atom or lone-pair routines commonly used to implement TIP4P and TIP5P. The similar behavior of both models using the DCM implementation and more widely used approaches therefore both validates the code and demonstrates the equivalence of the implementation in this context. Secondly, there is very good agreement for the polarizable, multipolar iAMOEBA results with (M)DCM, demonstrating that the close agreement in MEP and interaction energies yields correspondingly close $g(r)$ in simulations without the need for explicit multipoles, and using only generic functionality for off-centered charge sites in OpenMM.

Liquid density, heat of vaporization, isobaric heat capacity, isothermal compressibility, thermal expansion coefficient and self-diffusion coefficient at $298 \mathrm{~K}$ and 1 atm are summarized in Table 4. Again results for TIP $n \mathrm{P}$ models are compared with their DCM-representations and those for multipolar iAMOEBA are compared with those from (M)DCM and with experiment.

The calculated $\rho, \Delta H_{\text {vap }}$ and $D$ of $\operatorname{TIP} n \mathrm{P}$ waters are essentially identical from both sets of simulations. $C_{p}, \kappa$ and $\alpha$ values of water from the various models vary within \pm 1 unit, although for TIP4P $\alpha$ is somewhat overestimated, by about $30 \%$. As shown in the next section, this discrepancy seems to originate from noise in the data rather than a physical effect, as performance across a range of temperatures shows closer agreement and the other 
models also show local discrepancies at certain temperatures (Figure 7c). Agreement for the simulations with iAMOEBA and its (M)DCM representations is also very good, with the largest discrepancy being the slightly larger value for $\kappa$ with the DCM representation. Figure $7 \mathrm{~b}$ again shows this discrepancy is significantly smaller than the difference between different models.

The range of dynamic properties examined provides confidence that the microscopic structure and dynamics of the solvent are preserved when moving from a full multipolar description to the simpler distributed charge models, also after reducing the number of charges in the MDCM. All iAMOEBA results additionally agree well with experiment, consistent with earlier findings. ${ }^{32}$ It should also be noted that the data presented are for the simulation conditions described above, and hence certain deviations from previously published results are to be expected. For example $\Delta H_{\text {vap }}$ is $6 \%$ larger than the originally published Monte Carlo (MC) data, ${ }^{31} \kappa$ values are roughly $30\left(10^{6} \mathrm{~atm}^{-1}\right)$ smaller for TIP3P and TIP4P compared to original MC data, ${ }^{31,52}$ and $D$ for both TIP3P and TIP4P is roughly $40 \%$ larger than previously published results. ${ }^{76}$ As has been highlighted previously, ${ }^{61}$ small differences in simulation conditions can have a significant impact on the results from bulk simulations.

\subsection{Temperature Dependence of $\mathrm{H}_{2} \mathrm{O}$ Thermodynamic Properties}

As a more exacting test of the various water models and their (M)DCM representations, the density $\rho$, enthalpy of vaporization $\Delta H_{\text {vap }}$, isobaric heat capacity $C_{p}$, isothermal compressibility $\kappa$, and thermal expansion coefficient $\alpha$ were studied as a function of temperature $T$ between $235.5 \mathrm{~K}$ and $350 \mathrm{~K}$. Corresponding property vs. $T$ profiles are presented alongside reference experimental data in Figure 7 . In all cases the TIP $n \mathrm{P}$ models and their DCM representations agree very favourably. The same applies to iAMOEBA with the exception of $\kappa$ using the DCM representation for which a small shift is visible. 
Table 4: Bulk properties of liquid water at $298 \mathrm{~K}$ and $1 \mathrm{~atm}$; density $\rho\left(\mathrm{g} \mathrm{cm}^{-3}\right)$, enthalpy of vaporization $\Delta H_{\text {vap }}(\mathrm{kcal} / \mathrm{mol})$, isobaric heat capacity $C_{p}\left(\mathrm{cal} \mathrm{mol}^{-1} \mathrm{~K}^{-1}\right)$, isothermal compressibility $\kappa\left(10^{6} \mathrm{~atm}^{-1}\right)$, thermal expansion coefficient $\alpha\left(10^{5} \mathrm{~K}^{-1}\right)$ and self-diffusion coefficient $D\left(10^{-5} \mathrm{~cm}^{2} \mathrm{~s}^{-1}\right)$. "DCM" denotes the new DCM code and framework were used in place of standard routines.

\begin{tabular}{|c|c|c|c|c|c|c|}
\hline & $\rho$ & $\Delta H_{\mathrm{vap}}$ & $C_{p}$ & $\kappa$ & $\alpha$ & $D$ \\
\hline $\mathrm{TIP} 3 \mathrm{P}^{c}$ & 1.0266 & 11.04 & 12.9 & 23.1 & 76.8 & 3.9 \\
\hline TIP3P/DCM & 1.0264 & 11.04 & 12.9 & 22.4 & 75.6 & 3.9 \\
\hline TIP4P & 1.0090 & 11.15 & 15.6 & 22.7 & 35.3 & 2.3 \\
\hline TIP4P/DCM & 1.0082 & 11.16 & 15.5 & 23.3 & 44.9 & 2.3 \\
\hline TIP5P & 0.9848 & 10.73 & 24.2 & 28.8 & 30.9 & 2.2 \\
\hline TIP5P/DCM & 0.9842 & 10.73 & 24.8 & 30.0 & 30.0 & 2.2 \\
\hline iAMOEBA/OpenMM & 0.9977 & 10.91 & 17.8 & 40.7 & 23.3 & 1.9 \\
\hline iAMOEBA/DCM & 0.9916 & 10.85 & 18.3 & 51.0 & 25.8 & 2.0 \\
\hline iAMOEBA/MDCM10 & 0.9964 & 10.76 & 17.9 & 41.5 & 28.9 & 2.2 \\
\hline Exp..$^{a}$ & 0.9965 & 10.51 & 18.0 & 45.8 & 25.7 & $2.3^{b}$ \\
\hline
\end{tabular}

${ }^{a}$ Ref.; ${ }^{77 b}$ Ref.; ${ }^{78 c}$ using conventional TIP3P in CHARMM;

Liquid density: The dependence of bulk density $\rho$ on $T$ is shown in Figure 7a. In all cases there is close agreement between the original models and their (M)DCM representations, and much closer than the agreement between the different models. The multipolar and MDCM iAMOEBA descriptions are almost indistinguishable from the experimental curve, while the DCM description is also very close.

Isothermal compressibility and thermal expansion coefficient: Plots of isothermal compressibility $\kappa(T)$, and thermal expansion coefficient $\alpha(T)$ as a function of temperature are also included in Figures $7 \mathrm{~b}$ and c. (M)DCM representations of TIP $n \mathrm{P}$ again agree well with reference data, and more closely than the models agree with one another. The same is true for iAMOEBA, although some shift is visible in $\kappa$ for the DCM representation.

Relative to the experimental results, the additional detail in the iAMOEBA model, well 


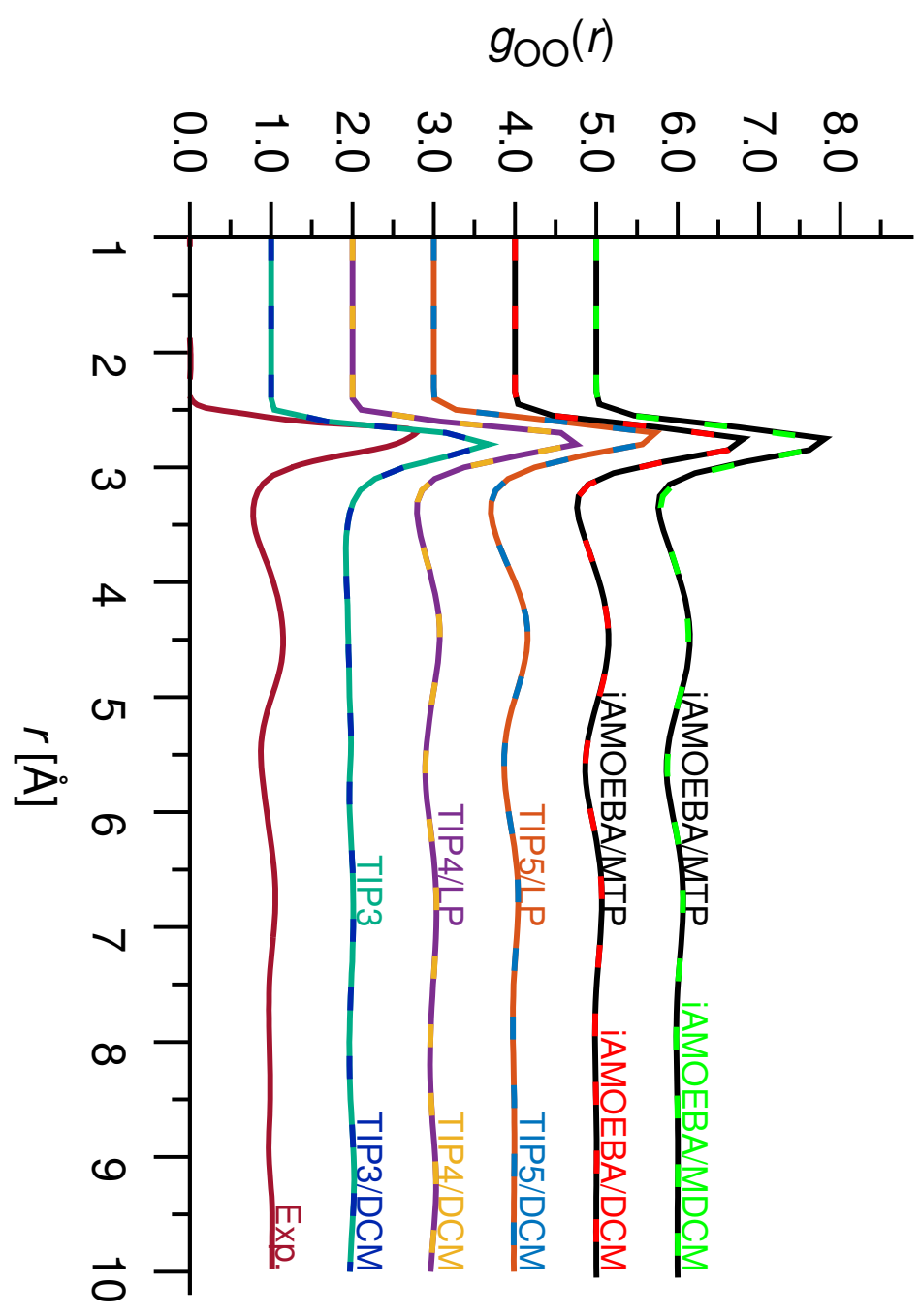

Figure 6: Radial distribution functions $(\mathrm{RDF}) g_{\mathrm{OO}}(r)$ of iAMOEBA and TIP $n \mathrm{P}$ $\mathrm{H}_{2} \mathrm{O}$ at $298 \mathrm{~K}$ and $1 \mathrm{~atm}$ using standard routines (LP) and DCM functionality in CHARMM (TIP $n \mathrm{P}$ ), and multipolar (MTP) iAMOEBA and (M)DCM implementations of iAMOEBA in OpenMM. Experimental neutron diffraction data from Ref. ${ }^{66}$ Successive curves are offset 1 unit along the $y$-axis for clarity.

encapsulated using distributed charges, affords a consistently accurate performance across the full range of $T$. 


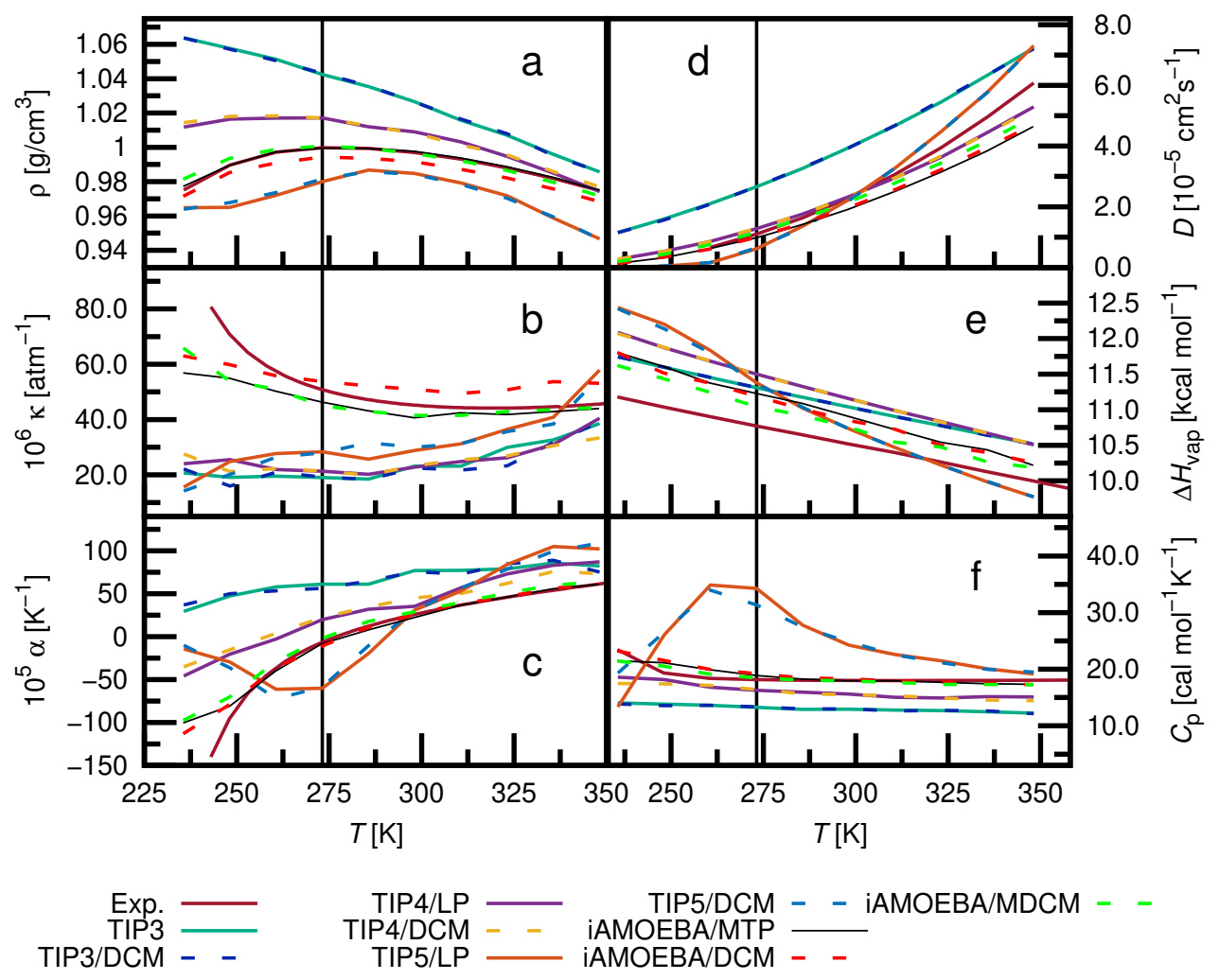

Figure 7: Experimental and computed densities $\rho$, diffusion coefficients $D$, isothermal compressibilities $\kappa$, enthalpies of vaporization $\Delta H_{\text {vap }}$, thermal expansion coefficients $\alpha$ and heat capacities $C_{\mathrm{p}}$ of liquid water using TIP $n \mathrm{P}$ and iAMOEBA water models as a function of temperature at 1 atm Exp. data from Refs. ${ }^{77-81}$ Experimental $\rho$ data are obscured by the iAMOEBA/MTP (multipolar iAMOEBA) data. A vertical line at $273 \mathrm{~K}$ indicates zero Celsius.

Diffusivity: The self-diffusion coefficient is one of the most frequently examined transport properties of water in MD studies. ${ }^{61}$ It measures the mobility of water molecules in the H-bonded liquid water network, and is thus taken as an indication of the accuracy of the water interaction potential. The simulated $D$, over the range of temperatures studied here, is presented in Figure 7d.

Enthalpy of vaporization: The variation of vaporization enthalpy $\Delta H_{\text {vap }}$ with $T$ (Figure 7e) shows significant differences between the different models, but in each case there is good 
agreement between the DCM implementation and the existing codes, and between the multipolar, polarizable charge model and (M)DCM results.

Isobaric heat capacity: For $C_{p}(T)$, again iAMOEBA and its (M)DCM representations agree well with one another across a broad range of temperatures. Slightly more deviation is visible for the DCM implementation of the TIP5P model. This model represents a significant outlier, though, in its agreement with experiment and the remaining models other than at high $T$, which may lead to increased sensitivity in this property.

\subsection{MDCM for PhF}

Next, the (M)DCM parametrization is extended to solvated systems. As an example, fluorobenzene $(\mathrm{PhF})$ was chosen. In the past it has been demonstrated that for halogenated benzenes including detailed electrostatics is mandatory for quantitative simulations. ${ }^{58,82,83}$

The presence of a halogen atom in PhF with a weak "sigma hole", combined with the availability of an existing multipolar model ${ }^{58}$ make it another suitable choice to evaluate the impact of replacing multipolar terms with distributed charges. In this case previously published experimental ${ }^{84-86}$ and computed ${ }^{58}$ solvation enthalpies, vaporization enthalpies of the pure liquid and heat capacities were available for comparison.

As for water, the first step was to obtain suitable MDCMs fitted to a grid of MEP points generated by the pre-existing multipolar model. Models were fitted with between 12 and 36 charges, i.e. with an average of between 1 and 3 charges per atom, see Figure 8. As the underlying multipolar model contained 42 non-zero multipolar terms, all charge models again offer a notable decrease in computational complexity for subsequent simulations. While the visible noise in Figure 8 with increasing number of charges in the fit shows that further refinement of the fitting procedure is possible, for example by increasing the number of DE 
fitting generations or the number of fits performed, the generally systematic improvement is encouraging and offers the possibility to select an MDCM based on an optimal compromise between computational cost of simulations due to increased number of charges, and improved accuracy in the electrostatics.

It is also encouraging to see that with 13 charges, i.e. an average of 1.1 charges per atom, the RMSE has already dropped to $0.19 \mathrm{kcal} / \mathrm{mol}$ with a maximum absolute error across the grid of $1.14 \mathrm{kcal} / \mathrm{mol}$. With 18 charges the RMSE is $0.08 \mathrm{kcal} / \mathrm{mol}$ and the maximum absolute error is $0.38 \mathrm{kcal} / \mathrm{mol}$ and with an average of 3 charges per atom the accuracy reaches an RMSE of $0.02 \mathrm{kcal} / \mathrm{mol}$ and the maximum absolute error is $0.12 \mathrm{kcal} / \mathrm{mol}$.
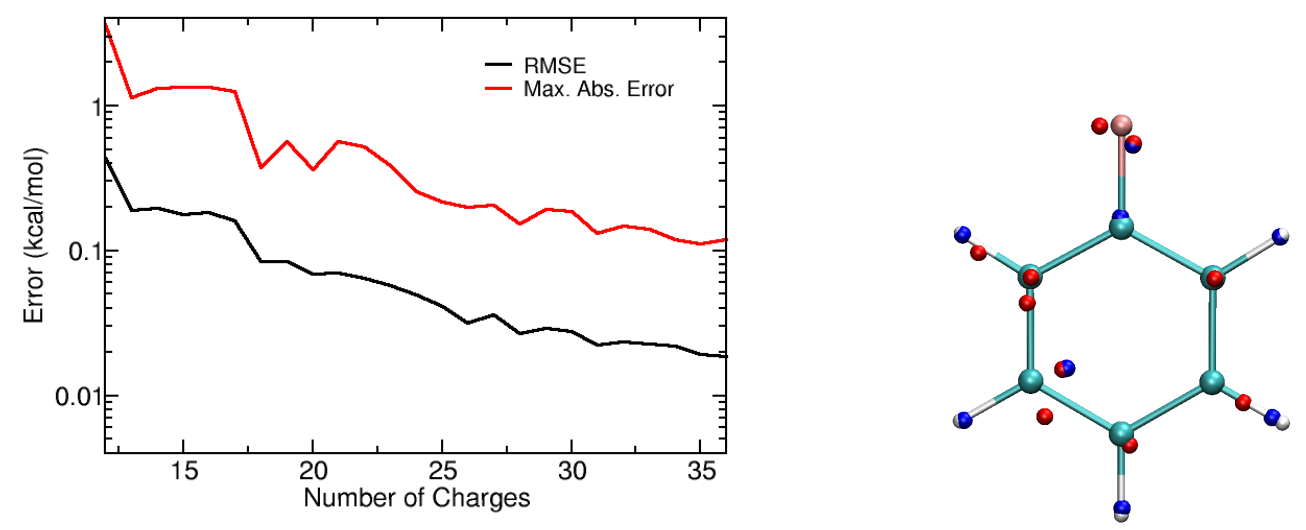

Figure 8: Left: RMSE and maximum absolute errors for fits of MDCMs to MEP reference data for $\mathrm{PhF}$ as a function of increasing number of charges. Right: location of the charges (blue positive, red negative) for the 22-charge model chosen for condensed phase simulations.

\subsection{Thermodynamic Properties of PhF}

After fitting MDCMs for PhF, the performance of the models in condensed phase simulations with respect to the performance of the original multipolar model was investigated. For this, the 22-charge MDCM was selected as it offered good accuracy (an RMSE of $0.06 \mathrm{kcal} / \mathrm{mol}$ 
across the electrostatic grid used for fitting and a maximum absolute error of $0.53 \mathrm{kcal} / \mathrm{mol}$ ) at modest computational cost with fewer than 2 charges on average per atom (Figure 8). Again, as no symmetry constraints were applied the charge positions are spatially asymmetric, but the close agreement in MEP with the symmetric underlying multipolar model demonstrates that the symmetry of the MEP is conserved.

The thermodynamic properties chosen for comparison with available experimental data were the density of pure liquid $\mathrm{PhF}$, the vaporization enthalpy of liquid $\mathrm{PhF}$ and the solvation free energy of a single PhF molecule in liquid water. As simulation conditions differed slightly from those used to fit the multipolar model ${ }^{58}$ a scaling factor of 1.1 was applied to the Lennard-Jones ' $\sigma$ ' and ' $\epsilon$ ' parameters of heavy atoms to recover the original performance of the multipolar model in describing these properties. The same scaling factor was applied to L-J parameters in MDCM simulations to allow direct comparison.

Results averaged over 8 independent simulations are shown in Table 5 . While both models agree quite well with experiment, there is again very good agreement between the multipolar and MDCM condensed-phase simulation results. That this agreement is again possible without refitting any other force-field terms and by fitting only to MEP grid-data highlights the equivalent performance of a modest number of distributed charges to a full multipoleexpansion truncated at quadrupole when describing the dynamics of these systems.

\subsection{Computational Efficiency}

While the results so far have demonstrated the equivalence in accuracy of distributed point charge and multipolar models in polarizable (iAMOEBA) and non-polarizable (PhF) MD simulations, any advantage over multipolar methods depends additionally on relative computational cost and ease of implementation into general-purpose MD codes. The second 
Table 5: Calculated free energy $\Delta G(\mathrm{kcal} / \mathrm{mol})$ of solvation of a single molecule of $\mathrm{PhF}$ in a TIP3P solvent box (averaged over 8 thermodynamic integration runs, with contributions from electrostatic and vdW terms), $\Delta H$ of vaporization $(\mathrm{kcal} / \mathrm{mol}$ ) and density at $300 \mathrm{~K}$ of pure liquid PhF. Results using an existing multipolar model for PhF are compared with results from a fitted MDCM with 22 charges. The standard deviation for each computed value is reported next to the number.

\begin{tabular}{|c|c|c|c|c|c|}
\hline & $\rho$ & $\overline{\Delta \Delta H_{\text {vap }}}$ & $\bar{c} \Delta G_{\text {solv,vdw }}^{c}$ & $\overline{\bar{c} \Delta G_{\text {solv,elec }}}$ & $\overline{\Delta \Delta G_{\text {solv,tot }}}$ \\
\hline Multipolar $^{a}$ & $0.90 \pm 0.01$ & $9.37 \pm 0.05$ & $2.21 \pm 0.13 \dagger$ & $-2.32 \pm 0.04$ & $-0.10 \pm 0.14$ \\
\hline MDCM & $0.90 \pm 0.01$ & $9.39 \pm 0.06$ & $2.21 \pm 0.13 \dagger$ & $-2.92 \pm 0.13$ & $-0.71 \pm 0.21$ \\
\hline Exp. & $1.02^{b}$ & $8.26^{c}$ & - & - & $-0.80^{d}$ \\
\hline
\end{tabular}

$\dagger$ The same 8 trajectories were used to evaluate Multipolar and MDCM vdW solvation energy contributions as solute electrostatics are zeroed in these calculations.

point has been demonstrated by introducing (M)DCMs for $\mathrm{H}_{2} \mathrm{O}$ into OpenMM by using only existing functionality. This suggests that (M)DCM can also be used in any other MD simulation code that provide appropriate functionality without undue additional technical effort.

The first point is more difficult to quantify in an objective manner as it depends on factors including hardware (CPU or GPU specifications, hardware optimization for important terms such as inverse square roots), compiler details, algorithmic efficiency, size of the simulated system, treatment of electrostatics (PME, cut-off distances), numerical precision and others. A rigorous treatment falls beyond the scope of the current work, but further to a brief analysis presented previously, ${ }^{26}$ analyses and timings for the simulations discussed here are given in section 10 of the SI. The observation is that MDCMs outperform MTPs, ranging from $\sim 20 \%$ for water to a factor of three for PhF. However, additional analysis for other molecules and types of simulations is required to yield conclusive results. 


\section{Conclusions}

(M)DCM has been improved in accuracy, generalized and extended to include polarizability and applied to condensed phase simulations. Its performance to capture multipolar interactions within a single point charge-based implementation has been demonstrated by comparing various condensed phase properties of water and solvated $\mathrm{PhF}$ with established multipolar representations. Specifically, the distributed charge framework (including distribution of torques, local axis system description, definition of cut-offs) maintains the original bulk simulation properties for traditional force field models such as TIP3P, models with one or more off-centered charges such as TIP4P and TIP5P, and multipolar models such as the recently developed iAMOEBA and a multipolar description of PhF. Although there are different possible ways to define axis systems (Figure 1) and to distribute the torques, the close agreement and consistency of the results from the MD simulations for eight condensed phase properties using the different models indicates that these choices are not critical to describing the dynamics of the system. This is not self-evident and a gratifying aspect of the present work.

The use of point charges in place of atom-centered multipoles removes the need for computationally expensive higher-order multipole terms to yield accurate and efficient dynamics. Furthermore, machine-learning reduces the number of charges necessary to a minimal set and the method has been combined with isotropic polarization and integrated with familiar tools such as barostats and thermodynamic integration routines to enable straightforward use of the models under widely encountered condensed-phase simulation conditions. The compatibility of the approach with an unmodified release of OpenMM and with all of the force field models tested here shows that distributed charges are a versatile tool for the development of next-generation force fields and multi-level approaches, and also offer an alternative to explicit multipolar terms of existing force fields. Beyond the simulation packages presented 
already, widely used codes such as Amber $^{1}$ also offer functionality for off-centered charges that could be combined with (M)DCM without further modification or prohibitive computational cost.

While the current work focused on directly comparing simulation results from (M)DCM with multipolar representations without refitting remaining parameters for direct comparison of the implementations, a generic (M)DCM force field, fitted to condensed phase experiments offers additional opportunities for quantitative simulations. This can be envisaged within the framework of an existing fitting environment ${ }^{44}$ and will be of particular interest when balancing accuracy (i.e. the number of charges per atom) and speed for specific applications. As was shown here it is possible by increasing the number of charges used in fitting to systematically improve the accuracy of the electrostatic interactions, allowing fine tuning of the computational cost of adding more charges and a corresponding improvement in the description of the electrostatic interaction. In this way, the present work opens up the possibility for custom-made MDCM force fields with calibrated accuracy to encapsulate the physics of a given application.

\section{Supplementary Material}

See supplementary material for an illustration of the complexity of multipolar versus distributed charge interaction terms, for parameter files with (M)DCM charge positions and magnitudes, for sample input files used to run (M)DCM in OpenMM, for radial distribution functions from different water model simulations, for a discussion of the relationship between errors in the electrostatic interaction energy and fitting errors in the MEP, for an analysis of the impact of broken symmetry in MDCMs on interaction energies, and for a discussion of relative computational costs of MDCMs and multipole moments. The code used to fit the 
MDCMs presented here is available freely at https://github.com/MMunibas/MDCM.

\section{ACKNOWLEDGEMENT}

The authors acknowledge financial support from the Swiss National Foundation for Research for their support within the NCCR MUST program and project 200021-117810.

\section{REFERENCES}

(1) Case, D. A.; Cheatham, T. E.; Darden, T.; Gohlke, H.; Luo, R.; Merz, K. M.; Onufriev, A.; Simmerling, C.; Wang, B.; Woods, R. J. The Amber biomolecular simulation programs. J. Comput. Chem. 2005, 26, 1668-1688.

(2) Jorgensen, W. L.; Tirado-Rives, J. Molecular modeling of organic and biomolecular systems using BOSS and MCPRO. J. Comput. Chem. 2005, 26, 1689-1700.

(3) Van Der Spoel, D.; Lindahl, E.; Hess, B.; Groenhof, G.; Mark, A. E.; Berendsen, H. J. GROMACS: fast, flexible, and free. J. Comput. Chem. 2005, 26, 1701-1718.

(4) Christen, M.; Hünenberger, P. H.; Bakowies, D.; Baron, R.; Bürgi, R.; Geerke, D. P.; Heinz, T. N.; Kastenholz, M. A.; Kräutler, V.; Oostenbrink, C.; Peter, C.; Trzesniak, D.; Gunsteren, W. F. V. The GROMOS software for biomolecular simulation: GROMOS05. J. Comput. Chem. 2005, 26, 1719-1751.

(5) MacKerell, Jr., A. D.; Brooks, B.; Brooks, III, C. L.; Roux, B. N.; Won, Y.; Karplus, M. In Encyclopedia of Computational Chemistry, 1st ed.; Schleyer, P. v. R., Ed.; John Wiley and Sons, Ltd.: New York, NY, 1998; pp 271-277.

(6) Stone, A. J. The Theory of Intermolecular Forces; Oxford University Press: Oxford, U.K., 2000. 
(7) Plattner, N.; Meuwly, M. The Role of Higher CO-Multipole Moments in Understanding the Dynamics of Photodissociated Carbonmonoxide in Myoglobin. Biophys. J. 2008, 94, 2505-2515.

(8) Cardamone, S.; Hughes, T. J.; Popelier, P. L. Multipolar electrostatics. Phys. Chem. Chem. Phys. 2014, 16, 10367-10387.

(9) Ren, P.; Ponder, J. W. Polarizable atomic multipole water model for molecular mechanics simulation. J. Phys. Chem. B 2003, 107, 5933-5947.

(10) Ponder, J. W.; Wu, C.; Ren, P.; Pande, V. S.; Chodera, J. D.; Schnieders, M. J.; Haque, I.; Mobley, D. L.; Lambrecht, D. S.; DiStasio Jr, R. A.; Head-Gordon, M.; Clark, G. N. I.; Johnson, M. E.; Head-Gordon, T. Current status of the AMOEBA polarizable force field. J. Phys. Chem. B 2010, 114, 2549-2564.

(11) Ren, P.; Wu, C.; Ponder, J. W. Polarizable atomic multipole-based molecular mechanics for organic molecules. J. Chem. Theory Comput. 2011, 7, 3143-3161.

(12) Laury, M. L.; Wang, L.-P.; Pande, V. S.; Head-Gordon, T.; Ponder, J. W. Revised parameters for the AMOEBA polarizable atomic multipole water model. J. Phys. Chem. $B 2015$,

(13) Gresh, N.; Cisneros, G. A.; Darden, T. A.; Piquemal, J.-P. Anisotropic, polarizable molecular mechanics studies of inter-and intramolecular interactions and ligandmacromolecule complexes. A bottom-up strategy. J. Chem. Theory Comput. 2007, 3, 1960-1986.

(14) Piquemal, J.-P.; Williams-Hubbard, B.; Fey, N.; Deeth, R. J.; Gresh, N.; GiessnerPrettre, C. Inclusion of the ligand field contribution in a polarizable molecular mechanics: SIBFA-LF. J. Comput. Chem. 2003, 24, 1963-1970. 
(15) Gresh, N.; Claverie, P.; Pullman, A. Theoretical studies of molecular conformation. Derivation of an additive procedure for the computation of intramolecular interaction energies. Comparison with ab initio SCF computations. Theor. Chim. Acta 1984, 66, $1-20$.

(16) Gresh, N. Energetics of $\mathrm{Zn} 2+$ binding to a series of biologically relevant ligands: A molecular mechanics investigation grounded on ab initio self-consistent field supermolecular computations. J. Comput. Chem. 1995, 16, 856-882.

(17) Piquemal, J.-P.; Gresh, N.; Giessner-Prettre, C. Improved formulas for the calculation of the electrostatic contribution to the intermolecular interaction energy from multipolar expansion of the electronic distribution. J. Phys. Chem. A 2003, 107, 10353-10359.

(18) Popelier, P. L. QCTFF: On the construction of a novel protein force field. Int. J. Quantum Chem. 2015, 115, 1005-1011.

(19) Gordon, M. S.; Fedorov, D. G.; Pruitt, S. R.; Slipchenko, L. V. Fragmentation methods: a route to accurate calculations on large systems. Chem. Rev. 2012, 112, 632-672.

(20) Cisneros, G. A. Application of gaussian electrostatic model (GEM) distributed multipoles in the AMOEBA force field. J. Chem. Theory Comput. 2012, 8, 5072-5080.

(21) Duke, R. E.; Starovoytov, O. N.; Piquemal, J.-P.; Cisneros, G. A. GEM*: A molecular electronic density-based force field for molecular dynamics simulations. J. Chem. Theory Comput. 2014, 10, 1361-1365.

(22) Engkvist, O.; Åstrand, P.-O.; Karlström, G. Accurate intermolecular potentials obtained from molecular wave functions: Bridging the gap between quantum chemistry and molecular simulations. Chem. Rev. 2000, 100, 4087-4108.

(23) Handley, C. M.; Hawe, G. I.; Kell, D. B.; Popelier, P. L. Optimal construction of a 
fast and accurate polarisable water potential based on multipole moments trained by machine learning. Phys. Chem. Chem. Phys. 2009, 11, 6365-6376.

(24) Kramer, C.; Gedeck, P.; Meuwly, M. Atomic multipoles: Electrostatic potential fit, local reference axis systems, and conformational dependence. J. Comput. Chem. 2012, 33, 1673-1688.

(25) Kramer, C.; Gedeck, P.; Meuwly, M. Multipole-based force fields from ab initio interaction energies and the need for jointly refitting all intermolecular parameters. J. Chem. Theory Comput. 2013, 9, 1499-1511.

(26) Devereux, M.; Raghunathan, S.; Fedorov, D. G.; Meuwly, M. A Novel, computationally efficient multipolar model employing distributed charges for molecular dynamics simulations. J. Chem. Theory Comput. 2014, 10, 4229-4241.

(27) Unke, O. T.; Devereux, M.; Meuwly, M. Minimal distributed charges: Multipolar quality at the cost of point charge electrostatics. J. Chem. Phys. 2017, 147, 161712.

(28) Brooks, B. R.; Bruccoleri, R. E.; Olafson, B. D.; States, D. J.; Swaminathan, S.; Karplus, M. CHARMM: a program for macromolecular energy, minimization and dynamics calculations. J. Comput. Chem. 1983, 4, 187-217.

(29) Eastman, P. et al. OpenMM 4: A Reusable, Extensible, Hardware Independent Library for High Performance Molecular Simulation. J. Chem. Theory Comput. 2013, 9, 461469.

(30) Merz, K. M. Using Quantum Mechanical Approaches to Study Biological Systems. Acc. Chem. Res. 2014, 47, 2804-2811.

(31) Jorgensen, W. L.; Chandrasekhar, J.; Madura, J. D.; Impey, R. W.; Klein, M. L. Comparison of simple potential functions for simulating liquid water. J. Chem. Phys. 1983, 79, 926-935. 
(32) Wang, L.-P.; Head-Gordon, T.; Ponder, J. W.; Ren, P.; Chodera, J. D.; Eastman, P. K.; Martinez, T. J.; Pande, V. S. Systematic improvement of a classical molecular model of water. J. Phys. Chem. B 2013, 117, 9956-9972.

(33) Price, S. S. L. Quantifying intermolecular interactions and their use in computational crystal structure prediction. Cryst. Eng. Comm. 2004, 6, 344-353.

(34) Stone, A. J. Distributed Multipole Analysis: Stability for Large Basis Sets. J. Chem. Theory Comput. 2005, 1, 1128-1132.

(35) Stone, A.; Alderton, M. Distributed multipole analysis. Mol. Phys. 1985, 56, 10471064 .

(36) Spackman, M. A. A simple quantitative model of hydrogen bonding. J. Chem. Phys. 1986, 85, 6587-6601.

(37) Misquitta, A. J.; Stone, A. J.; Fazeli, F. Distributed Multipoles from a Robust BasisSpace Implementation of the Iterated Stockholder Atoms Procedure. J. Chem. Theory Comput. 2014, 10, 5405-5418.

(38) Devereux, M.; Gresh, N.; Piquemal, J.-P.; Meuwly, M. A Supervised Fitting Approach to Force Field Parametrization with Application to the SIBFA Polarizable Force Field. J. Comput. Chem. 2014, 35, 1577-1591.

(39) Gao, Q.; Yokojima, S.; Fedorov, D. G.; Kitaura, K.; Sakurai, M.; Nakamura, S. Octahedral point-charge model and its application to fragment molecular orbital calculations of chemical shifts. Chem. Phys. Lett. 2014, 593, 165-173.

(40) Storn, R.; Price, K. Differential Evolution - A Simple and Efficient Heuristic for Global Optimization over Continuous Spaces. J. Glob. Optim. 1997, 11, 341-359.

(41) Straatsma, T. P.; McCammon, J. A. Molecular Dynamics Simulations with Interaction 
Potentials Including Polarization Development of a Noniterative Method and Application to Water. Molecular Simulation 1990, 5, 181-192.

(42) Halgren, T. A. The representation of van der Waals (vdW) interactions in molecular mechanics force fields: potential form, combination rules, and vdW parameters. J. Am. Chem. Soc. 1992, 114, 7827-7843.

(43) Devereux, M.; Unke, O.; Bereau, T. MDCM Repository. 2020; https://github.com/ MMunibas/MDCM.

(44) Hédin, F.; El Hage, K.; Meuwly, M. A Toolkit to Fit Nonbonded Parameters from and for Condensed Phase Simulations. J. Chem. Inf. Model. 2016, 56, 1479-1489.

(45) Eastman, P.; Swails, J.; Chodera, J. D.; McGibbon, R. T.; Zhao, Y.; Beauchamp, K. A.; Wang, L.-P.; Simmonett, A. C.; Harrigan, M. P.; Stern, C. D.; Wiewiora, R. P.; Brooks, B. R.; Pande, V. S. OpenMM 7: Rapid development of high performance algorithms for molecular dynamics. PLOS Comp. Biol. 2017, 13, 1-17.

(46) van Gunsteren, W.; Berendsen, H. Algorithms for macromolecular dynamics and constraint dynamics. Mol. Phys. 1977, 34, 1311-1327.

(47) Andersen, H. C. Molecular dynamics simulations at constant pressure and/or temperature. J. Chem. Phys. 1980, 72, 2384-2393.

(48) Melchionna, S.; Ciccotti, G.; Lee Holian, B. Hoover NPT dynamics for systems varying in shape and size. Mol. Phys. 1993, 78, 533-544.

(49) Martyna, G. J.; Tobias, D. J.; Klein, M. L. Constant pressure molecular dynamics algorithms. J. Chem. Phys. 1994, 101, 4177-4189.

(50) Nosé, S. A molecular dynamics method for simulations in the canonical ensemble. Mol. Phys. 1984, 52, 255-268. 
(51) Hoover, W. G. Canonical dynamics: equilibrium phase-space distributions. Phys. Rev. A 1985, 31, 1695.

(52) Jorgensen, W. L.; Jenson, C. Temperature dependence of TIP3P, SPC, and TIP4P water from NPT Monte Carlo simulations: Seeking temperatures of maximum density. J. Comput. Chem. 1998, 19, 1179-1186.

(53) Mahoney, M. W.; Jorgensen, W. L. A five-site model for liquid water and the reproduction of the density anomaly by rigid, nonpolarizable potential functions. J. Chem. Phys. 2000, 112, 8910-8922.

(54) Baez, L. A.; Clancy, P. Existence of a density maximum in extended simple point charge water. J. Chem. Phys. 1994, 101, 9837-9840.

(55) Jorgensen, W. L.; Tirado-Rives, J. Potential energy functions for atomic-level simulations of water and organic and biomolecular systems. Proc. Natl. Acad. Sci. USA 2005, 102, 6665-6670.

(56) Steinbach, P. J.; Brooks, B. R. New spherical-cutoff methods for long-range forces in macromolecular simulation. J. Comput. Chem. 1994, 15, 667-683.

(57) Brooks, B. R. Computer code implemented in CHARMM. 1997.

(58) Bereau, T.; Kramer, C.; Meuwly, M. Leveraging Symmetries of Static Atomic Multipole Electrostatics in Molecular Dynamics Simulations. J. Chem. Theory Comput. 2013, 9, $5450-5459$.

(59) Kirkwood, J. G. Statistical Mechanics of Fluid Mixtures. J. Chem. Phys. 1935, 3, $300-313$.

(60) Singh, U. C.; Brown, F. K.; Bash, P. A.; Kollman, P. A. An approach to the application of free energy perturbation methods using molecular dynamics: applications to the transformations of methanol to ethane, oxonium to ammonium, glycine to alanine, and 
alanine to phenylalanine in aqueous solution and to $\mathrm{H} 3 \mathrm{O}+(\mathrm{H} 2 \mathrm{O}) 3$ to $\mathrm{NH} 4+(\mathrm{H} 2 \mathrm{O}) 3$ in the gas phase. J. Am. Chem. Soc. 1987, 109, 1607-1614.

(61) Mark, P.; Nilsson, L. Structure and dynamics of the TIP3P, SPC, and SPC/E water models at 298 K. J. Phys. Chem. A 2001, 105, 9954-9960.

(62) Rick, S. W. A reoptimization of the five-site water potential (TIP5P) for use with Ewald sums. J. Chem. Phys. 2004, 120, 6085-6093.

(63) Horn, H. W.; Swope, W. C.; Pitera, J. W.; Madura, J. D.; Dick, T. J.; Hura, G. L.; Head-Gordon, T. Development of an improved four-site water model for biomolecular simulations: TIP4P-Ew. J. Chem. Phys. 2004, 120, 9665-9678.

(64) Anderson, J. A.; Tschumper, G. S. Characterizing the potential energy surface of the water dimer with DFT: failures of some popular functionals for hydrogen bonding. $J$. Phys. Chem. A 2006, 110, 7268-7271.

(65) Maheshwary, S.; Patel, N.; Sathyamurthy, N.; Kulkarni, A. D.; Gadre, S. R. Structure and stability of water clusters $(\mathrm{H} 2 \mathrm{O}) \mathrm{n}, \mathrm{n}=8-20$ : An ab initio investigation. J. Phys. Chem. A 2001, 105, 10525-10537.

(66) Soper, A. The radial distribution functions of water and ice from 220 to $673 \mathrm{~K}$ and at pressures up to $400 \mathrm{MPa}$. Chem. Phys. 2000, 258, 121-137.

(67) Hura, G.; Sorenson, J. M.; Glaeser, R. M.; Head-Gordon, T. A high-quality X-ray scattering experiment on liquid water at ambient conditions. J. Chem. Phys. 2000, 113, 9140-9148.

(68) Fu, L.; Bienenstock, A.; Brennan, S. X-ray study of the structure of liquid water. J. Chem. Phys. 2009, 131, 234702.

(69) Leetmaa, M.; Wikfeldt, K. T.; Ljungberg, M. P.; Odelius, M.; Swenson, J.; Nilsson, A.; 
Pettersson, L. G. Diffraction and IR/Raman data do not prove tetrahedral water. J. Chem. Phys. 2008, 129, 084502.

(70) Wikfeldt, K. T.; Leetmaa, M.; Mace, A.; Nilsson, A.; Pettersson, L. G. Oxygen-oxygen correlations in liquid water: Addressing the discrepancy between diffraction and extended x-ray absorption fine-structure using a novel multiple-data set fitting technique. J. Chem. Phys. 2010, 132, 104513.

(71) Petkov, V.; Ren, Y.; Suchomel, M. Molecular arrangement in water: random but not quite. J. Phys.: Condens. Matter 2012, 24, 155102.

(72) Hura, G.; Russo, D.; Glaeser, R. M.; Head-Gordon, T.; Krack, M.; Parrinello, M. Water structure as a function of temperature from X-ray scattering experiments and ab initio molecular dynamics. Phys. Chem. Chem. Phys. 2003, 5, 1981-1991.

(73) Head-Gordon, T.; Hura, G. Water structure from scattering experiments and simulation. Chem. Rev. 2002, 102, 2651-2670.

(74) Soper, A. Joint structure refinement of x-ray and neutron diffraction data on disordered materials: application to liquid water. J. Phys.: Condens. Matter 2007, 19, 335206.

(75) Skinner, L. B.; Huang, C.; Schlesinger, D.; Pettersson, L. G.; Nilsson, A.; Benmore, C. J. Benchmark oxygen-oxygen pair-distribution function of ambient water from X-ray diffraction measurements with a wide Q-range. J. Chem. Phys. 2013, 138, 074506.

(76) Mahoney, M. W.; Jorgensen, W. L. Diffusion constant of the TIP5P model of liquid water. J. Chem. Phys. 2001, 114, 363-366.

(77) Wagner, W.; Pruß, A. The IAPWS formulation 1995 for the thermodynamic properties of ordinary water substance for general and scientific use. J. Phys. Chem. Ref. Data 2002, 31, 387-535. 
(78) Mills, R. Self-diffusion in normal and heavy water in the range 1-45. deg. J. Phys. Chem. 1973, r7, 685-688.

(79) Archer, D. G.; Carter, R. W. Thermodynamic properties of the $\mathrm{NaCl}+\mathrm{H} 2 \mathrm{O}$ system. 4. heat capacities of h2o and nacl (aq) in cold-stable and supercooled states. J. Phys. Chem. B 2000, 104, 8563-8584.

(80) Kell, G. S. Density, thermal expansivity, and compressibility of liquid water from 0. deg. to 150. deg.. Correlations and tables for atmospheric pressure and saturation reviewed and expressed on 1968 temperature scale. J. Chem. Eng. Data 1975, 20, 97-105.

(81) Gillen, K. T.; Douglass, D.; Hoch, M. Self-Diffusion in Liquid Water to $-31^{\circ}$ C. J. Chem. Phys. 1972, 57, 5117-5119.

(82) Jorgensen, W. L.; Schyman, P. Treatment of Halogen Bonding in the OPLS-AA Force Field: Application to Potent Anti-HIV Agents. J. Chem. Theory Comput. 2012, 8, 3895-3901, PMID: 23329896.

(83) Politzer, P.; Murray, J. S.; Clark, T. Halogen bonding: an electrostatically-driven highly directional noncovalent interaction. Phys. Chem. Chem. Phys. 2010, 12, 7748-7757.

(84) Wang, J.; Hou, T. Application of Molecular Dynamics Simulations in Molecular Property Prediction. 1. Density and Heat of Vaporization. J. Chem. Theory Comput. 2011, 7, 2151-2165, PMID: 21857814.

(85) Majer, V.; Svoboda, V. Enthalpies of Vaporization of Organic Compounds: A Critical Review and Data Compilation; Blackwell Scientific Publications: Oxford, U.K., 1985.

(86) Mobley, D. L.; Bayly, C. I.; Cooper, M. D.; Shirts, M. R.; Dill, K. A. Small Molecule Hydration Free Energies in Explicit Solvent: An Extensive Test of Fixed-Charge Atomistic Simulations. J. Chem. Theory Comput. 2009, 5, 350-358, PMID: 20150953. 


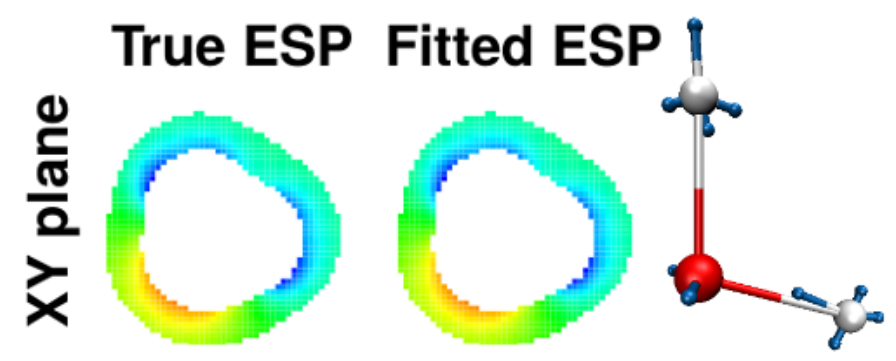

Figure 9: TOC graphic

47 


\title{
Supporting Information:
}

\section{Polarizable Multipolar Molecular Dynamics Using Distributed Point Charges}

\author{
Mike Devereux, Marco Pezzella, Shampa Raghunathan and Markus Meuwly* \\ Department of Chemistry, University of Basel, Klingelbergstrasse 80, CH-4056 Basel, \\ Switzerland \\ E-mail: m.meuwly@unibas.ch
}




\section{Contents}

1 Reduction in complexity using distributed charges S-3

2 (M)DCM Charge Models $\quad$ S-5

3 iAMOEBA DCM OpenMM force field definition file $\quad$ S-7

4 Error Reduction in MDCMs $\quad$ S-18

5 iAMOEBA MDCM OpenMM force field definition file S-20

6 Impact of Broken Symmetry in MDCMs $\quad$ S-27

7 O...O Radial Distribution Functions $\quad$ S-30

8 O...H Radial Distribution Functions $\quad$ S-31

$9 \quad$ H...H Radial Distribution Functions $\quad$ S-32

10 Computational Efficiency $\quad$ S-33

$\begin{array}{ll}\text { References } & \text { S-37 }\end{array}$ 


\section{Reduction in complexity using distributed charges}

The main benefit of using distributed charges over a truncated multipole expansion is that the electrostatic interaction energy between two multipolar atoms can be evaluated using a homogeneous set of charge-charge interaction terms in place of the heterogeneous terms between multipole moments of different ranks. I.e. all energy terms are of the form:

$$
V_{\mathrm{ab}}=\frac{q_{\mathrm{a}} q_{\mathrm{b}}}{R_{\mathrm{ab}}}
$$

where $R_{\mathrm{ab}}$ is the distance between atoms $a$ and $b$. By contrast, multipolar interaction terms are heterogeneous and especially higher rank terms are substantially more complex. The following example describes the interaction between multipole moments $Q_{l, m}$ on atoms $a$ and $d:$ S1 


$$
\begin{aligned}
V_{\mathrm{Q}_{00, \mathrm{a}} \mathrm{Q}_{00, \mathrm{~d}}} & =\frac{Q_{00, \mathrm{a}} Q_{00, \mathrm{~d}}}{r_{\mathrm{ad}}} \\
V_{\mathrm{Q}_{00, \mathrm{a}} \mathrm{Q}_{1 \mathrm{~m}, \mathrm{~d}}} & =\frac{Q_{00, \mathrm{a}} Q_{1 m, \mathrm{~d}} r_{\alpha}^{\mathrm{a}}}{r_{\mathrm{ad}}^{2}} \\
V_{\mathrm{Q}_{1 \mathrm{~m}, \mathrm{a}} \mathrm{Q}_{1 \mathrm{~m}, \mathrm{~d}}} & =\frac{Q_{1 m, \mathrm{a}} Q_{1 m, \mathrm{~d}}\left(3 r_{\alpha}^{\mathrm{a}} r_{\beta}^{\mathrm{d}}+c_{\alpha, \beta}\right)}{r_{\mathrm{ad}}^{3}} \\
V_{\mathrm{Q}_{20, \mathrm{a}} \mathrm{Q}_{00}, \mathrm{~d}} & =\frac{Q_{20, \mathrm{a}} Q_{00, \mathrm{~d}}\left(3 r_{z}^{\mathrm{a} 2}-1\right)}{2 r_{\mathrm{ad}}^{3}} \\
V_{\mathrm{Q}_{22 \mathrm{c}, \mathrm{a}} \mathrm{Q}_{00}, \mathrm{~d}} & =\frac{\sqrt{3} Q_{22 c, \mathrm{a}} Q_{00, \mathrm{~d}}\left(r_{x}^{\mathrm{a} 2}-r_{y}^{\mathrm{a} 2}\right)}{2 r_{\mathrm{ad}}^{3}} \\
V_{\mathrm{Q}_{20, \mathrm{a}} \mathrm{Q}_{1 \mathrm{~m}, \mathrm{~d}}} & =\frac{Q_{20, \mathrm{a}} Q_{1 m, \mathrm{~d}}\left(15 r_{z}^{\mathrm{a} 2} r_{\beta}^{\mathrm{d}}+6 c_{z A, \beta} r_{z}^{\mathrm{a}}-3 r_{\beta}^{\mathrm{d}}\right)}{2 r_{\mathrm{ad}}^{4}} \\
V_{\mathrm{Q}_{22 \mathrm{c}, \mathrm{a}} \mathrm{Q}_{1 \mathrm{~m}, \mathrm{~d}}} & =\frac{\sqrt{3} Q_{22 c, \mathrm{a}} Q_{1 m, \mathrm{~d}}\left(5 r_{\beta}^{\mathrm{d}}\left(r_{x}^{\mathrm{a} 2}-r_{y}^{\mathrm{a} 2}\right)+2 c_{x A, \beta} r_{x}^{\mathrm{a}}-2 c_{y A, \beta} r_{y}^{\mathrm{a}}\right)}{2 r_{\mathrm{ad}}^{4}} \\
V_{\mathrm{Q}_{20, \mathrm{a}} \mathrm{Q}_{20, \mathrm{~d}}} & =\frac{3 Q_{20, \mathrm{a}} Q_{20, \mathrm{~d}}\left(35 r_{z}^{\mathrm{a} 2} r_{z}^{\mathrm{d} 2}-5 r_{z}^{\mathrm{a} 2}-5 r_{z}^{\mathrm{d} 2}+20 r_{z}^{\mathrm{a}} r_{z}^{\mathrm{d}} c_{z A, z D}+2 c_{z A, z D}^{2}+1\right)}{4 r_{\mathrm{ad}}^{5}} \\
V_{\mathrm{Q}_{20, \mathrm{a}} \mathrm{Q}_{22 c, \mathrm{~d}}} & =\frac{\sqrt{3} Q_{20, \mathrm{a}} Q_{22 c, \mathrm{~d}}\left(35 r_{z}^{\mathrm{a} 2} r_{x}^{\mathrm{d}} r_{y}^{\mathrm{d}}-5 r_{x}^{\mathrm{d}} r_{y}^{\mathrm{d}}+10 r_{z}^{\mathrm{a}} r_{x}^{\mathrm{d}} c_{z A, y D}+10 r_{z}^{\mathrm{a}} r_{y}^{\mathrm{d}} c_{z A, x D}+2 c_{z A, x D} c_{z A, y D}\right)}{2 r_{\mathrm{ad}}^{5}}
\end{aligned}
$$

$$
\begin{aligned}
V_{\mathrm{Q}_{22 c, \mathrm{a}} \mathrm{Q}_{22 \mathrm{c}, \mathrm{d}}}=\frac{1}{4} & Q_{22 c, \mathrm{a}} Q_{22 c, \mathrm{~d}}\left(35 r_{x}^{\mathrm{a} 2} r_{x}^{\mathrm{d} 2}-35 r_{x}^{\mathrm{a} 2} r_{y}^{\mathrm{d} 2} r_{y}^{\mathrm{d}}-35 r_{y}^{\mathrm{a} 2} r_{x}^{\mathrm{d} 2}+35 r_{y}^{\mathrm{a} 2} r_{y}^{\mathrm{d} 2} r_{y}^{\mathrm{d}} r_{y}^{\mathrm{d}}\right. \\
& +20 r_{x}^{\mathrm{a}} r_{x}^{\mathrm{d}} c_{x A, x D}-20 r_{x}^{\mathrm{a}} r_{y}^{\mathrm{d}} c_{x A, y D}-20 r_{y}^{\mathrm{a}} r_{x}^{\mathrm{d}} c_{y A, x D}+20 r_{y}^{\mathrm{a}} r_{y}^{\mathrm{d}} c_{y A, y D} \\
& \left.+2 c_{x A, x D}^{2}-2 c_{x A, y D}^{2}-2 c_{y A, x D}^{2}+2 c_{y A, y D}^{2}\right) r_{\mathrm{ad}}^{-5}
\end{aligned}
$$

Here the multipole moment $Q_{1 m}$ is the dipole moment component $m(l=1)$. When $m=$ $0,1,2, \alpha=z, x, y$ respectively. $r_{\text {ad }}$ is the internuclear separation of atoms a and $\mathrm{d}, r_{\alpha}^{\mathrm{a}}$ is defined as the scalar product $\hat{\mathbf{e}}_{\alpha}^{\mathrm{a}} \cdot \hat{\mathbf{e}}_{\mathrm{ad}}$ where $\hat{\mathbf{e}}_{\alpha}^{\mathrm{a}}$ is a unit vector along the $\alpha$ axis of atom a $(\alpha=x, y, z)$. $\hat{\mathbf{e}}_{\text {ad }}$ is a unit vector in the direction from a to $\mathrm{d}$. $\beta=x, y, z$ for atom $\mathrm{d}, c_{\alpha, \beta}$ is the scalar product $\hat{\mathbf{e}}_{\alpha}^{\mathrm{a}} \cdot \hat{\mathbf{e}}_{\beta}^{\mathrm{d}}$.

In order to run molecular dynamics simulations the gradients of each of these terms must also be evaluated for every nonbonded atom pair at each simulation time step. 


\section{2 (M)DCM Charge Models}

Table S1: Octahedrally arranged DCM charges (a.u.) and their coordinates $(\AA)$ for $\mathrm{H}_{2} \mathrm{O}$ used in iAMOEBA model; atomic polarizabilities $\alpha$ in $\AA^{3}$. Coordinates are relative to local atomic axes. ${ }^{\text {S2 }}$

\begin{tabular}{cccccc}
\hline \hline & $X$ & $Y$ & $Z$ & $q$ & $\alpha$ \\
\hline $\mathbf{H}$ & -0.0956010671 & 0.0000000000 & 0.0090474640 & 1.6134817638 & \\
$q_{1}$ & 0.0000000000 & 0.0959947647 & 0.0000000000 & 0.2524281689 & \\
$q_{2}$ & 0.0956010671 & 0.0000000000 & -0.0090474640 & 2.0482594186 & \\
$q_{3}$ & -0.0086850918 & 0.0000000000 & -0.0995898760 & -2.2882340867 & \\
$q_{4}$ & 0.0000000000 & -0.0959947647 & 0.0000000000 & 0.2524281689 & \\
$q_{5}$ & 0.0086850918 & 0.0000000000 & 0.0995898760 & -1.5813534334 & \\
$q_{6}$ & & & & & 0.80636 \\
$\mathbf{O}$ & & & & \\
$q_{1}$ & -0.0575896814 & 0.0000000000 & -0.0800056071 & 2.0122163666 & \\
$q_{2}$ & 0.0000000000 & -0.0959947647 & 0.0000000000 & -3.1087375276 & \\
$q_{3}$ & 0.0575896814 & 0.0000000000 & 0.0800056071 & 2.0122163666 & \\
$q_{4}$ & -0.0768011943 & 0.0000000000 & 0.0599925231 & 1.0336186790 & \\
$q_{5}$ & 0.0000000000 & 0.0959947647 & 0.0000000000 & -3.1087375276 & \\
$q_{6}$ & 0.0768011943 & 0.0000000000 & -0.0599925231 & 0.5654036430 & \\
\hline $\mathbf{H}$ & & & & & 0.50484 \\
$q_{1}$ & 0.0956010671 & 0.0000000000 & 0.0090474640 & 1.6134817638 & \\
$q_{2}$ & 0.0000000000 & -0.0959947647 & 0.0000000000 & 0.2524281689 & \\
$q_{3}$ & -0.0956010671 & 0.0000000000 & -0.0090474640 & 2.0482594186 & \\
$q_{4}$ & 0.0086850918 & 0.0000000000 & -0.0995898760 & -2.2882340867 & \\
$q_{5}$ & 0.0000000000 & 0.0959947647 & 0.0000000000 & 0.2524281689 & \\
$q_{6}$ & -0.0086850918 & 0.0000000000 & 0.0995898760 & -1.5813534334 & \\
\hline \hline
\end{tabular}


Table S2: MDCM charges (a.u.) and their positions $(\AA)$ for 10-charge $\mathrm{H}_{2} \mathrm{O}$ model used for iAMOEBA; atomic polarizabilities $\alpha$ in $\AA^{3}$. Coordinates are relative to local atomic axes. ${ }^{\mathrm{S} 2}$

\begin{tabular}{cccccc}
\hline \hline & $X$ & $Y$ & $Z$ & $q$ & $\alpha$ \\
\hline $\mathbf{H}$ & & & & & 0.50484 \\
$q_{1}$ & 0.0209144768 & 0.0000004420 & 0.3473443817 & -0.1066619461 & \\
$q_{2}$ & -0.0452396324 & 0.0000004981 & -0.1651346312 & -0.9725811260 & \\
$q_{3}$ & -0.1905254480 & 0.0000021077 & -0.0528319716 & 0.5997075639 & \\
$q_{4}$ & 0.1496075438 & -0.0018910650 & -0.0565055370 & 0.8289103944 & \\
\hline $\mathbf{O}$ & & & & & 0.80636 \\
$q_{1}$ & 0.1009343674 & 0.4067419560 & -0.0294610237 & -0.2369290661 & \\
$q_{2}$ & 0.0881957994 & -0.3169912077 & -0.0203134193 & -0.3142376636 & \\
\hline $\mathbf{H}$ & & & & & 0.50484 \\
$q_{1}$ & 0.1659300202 & -0.0008225336 & 0.0275557737 & 0.5465716486 & \\
$q_{2}$ & -0.1837974669 & -0.0020877351 & -0.0477859191 & 0.6204782536 & \\
$q_{3}$ & 0.0519918506 & -0.0000018568 & -0.3764240427 & -0.6244927174 & \\
$q_{4}$ & 0.0355028536 & -0.0000020937 & 0.2009935462 & -0.3407653412 & \\
\hline \hline
\end{tabular}




\section{3 iAMOEBA DCM OpenMM force field definition file}

The following "XML" format force field definition file defines the iAMOEBA/DCM implementation for use in OpenMM. While dummy atom types could potentially be consolidated (all dummy atoms share the same mass and VDW, sites belonging to the same atom-type also have the same polarizability), the exhaustive approach taken here is convenient for development purposes.

$$
\begin{aligned}
& <\text { ForceField }> \\
& <\text { AtomTypes }> \\
& <\text { Type name="380" class="73" element="O" mass="15.999" / > } \\
& <\text { Type name="381" class="74" element="H" mass="1.008" / > } \\
& <\text { Type name="382" class }=" 75 " \text { mass }=" 0 " /> \\
& <\text { Type name="383" class }=" 76 " \text { mass }=" 0 " /> \\
& <\text { Type name="384" class }=" 77 " \text { mass }=" 0 " /> \\
& <\text { Type name }=" 385 " \text { class }=" 78 " \text { mass }=" 0 " /> \\
& <\text { Type name="386" class }=" 79 " \text { mass }=" 0 " /> \\
& <\text { Type name }=" 387 \text { " class }=" 80 " \text { mass }=" 0 " /> \\
& <\text { Type name }=" 388 " \text { class }=" 81 " \text { mass }=" 0 " /> \\
& <\text { Type name="389" class }=" 82 " \text { mass }=" 0 " /> \\
& <\text { Type name }=" 390 " \text { class }=" 83 " \text { mass }=" 0 " /> \\
& <\text { Type name="391" class }=" 84 " \text { mass }=" 0 " /> \\
& <\text { Type name="392" class="85" mass ="0" } /> \\
& <\text { Type name="393" class }=" 86 " \text { mass }=" 0 " /> \\
& <\text { Type name="394" class }=" 87 " \text { mass }=" 0 " /> \\
& <\text { Type name }=" 395 " \text { class }=" 88 " \text { mass }=" 0 " /> \\
& <\text { Type name="396" class }=" 89 " \text { mass }=" 0 " /> \\
& <\text { Type name="397" class }=" 90 " \text { mass }=" 0 " /> \\
& <\text { Type name="398" class="91" mass="0" } />
\end{aligned}
$$




$$
\begin{aligned}
& <\text { Type name="399" class="92" mass="0" / > } \\
& </ \text { AtomTypes }> \\
& <\text { Residues }> \\
& <\text { Residue name="HOH" }> \\
& <\text { Atom name }=" \text { O" type }=" 380 " /> \\
& <\text { Atom name }=" H 1 " \text { type }=" 381 " /> \\
& <\text { Atom name="H2" type="381" / > } \\
& <\text { Atom name="M1" type="382" / > } \\
& <\text { Atom name="M2" type="383" / > } \\
& <\text { Atom name="M3" type="384" / > } \\
& <\text { Atom name }=" \mathrm{M} 4 " \text { type }=" 385 " /> \\
& <\text { Atom name="M5" type }=" 386 " /> \\
& <\text { Atom name="M6" type="387" / > } \\
& <\text { Atom name="M7" type="388" / > } \\
& <\text { Atom name="M8" type="389" /> } \\
& <\text { Atom name="M9" type="390" / > } \\
& <\text { Atom name="M10" type="391" / > } \\
& <\text { Atom name="M11" type="392" / > } \\
& <\text { Atom name="M12" type="393" / > } \\
& <\text { Atom name="M13" type="394" / > } \\
& <\text { Atom name="M14" type="395" / > } \\
& <\text { Atom name="M15" type="396" /> } \\
& <\text { Atom name="M16" type="397" / > } \\
& <\text { Atom name="M17" type="398" / > } \\
& <\text { Atom name="M18" type="399" / > } \\
& <\text { Bond from }=" 0 " \text { to }=" 2 " /> \\
& <\text { Bond from }=" 0 " \text { to }=" 1 " />
\end{aligned}
$$


$<!--H 1-$ atom charges and positions $-->$

$<$ VirtualSite type="localCoords" index="3" atom $1=" 0 "$ atom $2=" 1 "$ atom $3=" 2 "$ wo1= "0.0" wo2="1.0" wo3=" $0.0 "$ wx $1="-1.0 "$ wx $2=" 1.0 "$ wx $3=" 0.0 "$ wy $1="-1.0 "$ wy2 $=" 0.0 "$ wy3 $3=" 1.0 " \mathrm{p} 1="-0.000905 " \mathrm{p} 2="-0.009551 " \mathrm{p} 3="-0.000000 " />$

$<$ VirtualSite type="localCoords" index="4" atom $1=" 0 "$ atom $2=" 1 "$ atom $3=" 2 "$ wo1= "0.0" wo2 $=" 1.0 "$ wo3 $=" 0.0 "$ wx $1="-1.0 "$ wx $2=" 1.0 "$ wx $3=" 0.0 "$ wy $1="-1.0 "$ wy2 $=" 0.0 "$ wy3 $=" 1.0 " \mathrm{p} 1="-0.000000 " \mathrm{p} 2="-0.000000 " \mathrm{p} 3="-0.009591 " />$

$<$ VirtualSite type="localCoords" index="5" atom $1=" 0 "$ atom $2=" 1 "$ atom $3=" 2 "$ wo1= "0.0" wo2="1.0" wo3=" $0.0 "$ wx $1="-1.0 "$ wx $2=" 1.0 "$ wx $3=" 0.0 "$ wy $1="-1.0 "$ wy 2 $=" 0.0 "$ wy3 $=" 1.0 " \mathrm{p} 1="-0.000905 " \mathrm{p} 2="-0.009551 " \mathrm{p} 3="-0.000000 " />$

$<$ VirtualSite type="localCoords" index $=" 6 "$ atom $1=" 0 "$ atom $2=" 1 "$ atom $3=" 2 "$ wo1= "0.0" wo2="1.0" wo3=" $0.0 "$ wx $1="-1.0 "$ wx $2=" 1.0 "$ wx $3=" 0.0 "$ wy $1="-1.0 "$ wy 2 $=" 0.0 "$ wy3 $=" 1.0 " \mathrm{p} 1="-0.009959 " \mathrm{p} 2="\lrcorner 0.000868 " \mathrm{p} 3="\llcorner 0.000000 " />$

$<$ VirtualSite type="localCoords" index="7" atom1="0" atom $2=" 1 "$ atom $3=" 2 "$ wo1= $" 0.0 "$ wo2 $=" 1.0 "$ wo3 $3=0.0 "$ wx $1="-1.0 "$ wx $2=" 1.0 "$ wx $3=" 0.0 "$ wy $1="-1.0 "$ wy 2 $=" 0.0 "$ wy3 $=" 1.0 " \mathrm{p} 1="-0.000000 " \mathrm{p} 2="-0.000000 " \mathrm{p} 3="-0.009591 " />$

$<$ VirtualSite type="localCoords" index=" 8" atom $1=" 0 "$ atom $2=" 1$ " atom $3=" 2 "$ wo1= " $0.0 "$ wo2 $=" 1.0 "$ wo3 $=" 0.0 "$ wx $1="-1.0 "$ wx $2=" 1.0 "$ wx $3=" 0.0 "$ wy $1="-1.0 "$ wy 2 $=" 0.0 "$ wy3 $=" 1.0 " \mathrm{p} 1="-0.009959 " \mathrm{p} 2="-0.000868 " \mathrm{p} 3="-0.000000 " />$ $<!--O$-atom charges and positions $-->$

$<$ VirtualSite type="localCoords" index="9" atom $1=" 0 "$ atom $2=" 1 "$ atom $3=" 2 "$ wo1= $" 1.0 "$ wo2 $=" 0.0 "$ wo3 $=" 0.0 "$ wx $1="-1.0 "$ wx $2=" 1.0 "$ wx $3=" 0.0 "$ wy $1="-1.0 "$ wy 2 $=" 0.0 "$ wy3 $3=1.0 " \mathrm{p} 1="-0.008010 " \mathrm{p} 2="-0.005742 " \mathrm{p} 3="-0.000000 " />$ $<$ VirtualSite type="localCoords" index="10" atom $1=" 0 "$ atom $2=" 1$ " atom $3=" 2$ " wo1 $=" 1.0 "$ wo2 $=" 0.0 "$ wo3 $=" 0.0 "$ wx1 $="-1.0 "$ wx $2=" 1.0 "$ wx $3=" 0.0 "$ wy $1="-1.0 "$ wy2="0.0" wy3="1.0" p1=" $0.000000 " \mathrm{p} 2="-0.000000 " \mathrm{p} 3="-0.009591 " />$ 
$<$ VirtualSite type="localCoords" index="11" atom $1=" 0 "$ atom $2=" 1$ " atom $3=" 2 "$ wo1 $=" 1.0 "$ wo $2=" 0.0 "$ wo3 $=" 0.0 "$ wx $1="-1.0 "$ wx $2=" 1.0 "$ wx $3=" 0.0 "$ wy $1="-1.0 "$ wy2="0.0" wy3="1.0" p1=" $0.008010 " \mathrm{p} 2="-0.005742 " \mathrm{p} 3="-0.000000 " />$ $<$ VirtualSite type="localCoords" index="12" atom1="0" atom2="1" atom3="2" wo1 $=" 1.0 "$ wo $2=" 0.0 "$ wo3 $=" 0.0 "$ wx $1="-1.0 " \mathrm{wx} 2=" 1.0 " \mathrm{wx} 3=" 0.0 " \mathrm{wy} 1="-1.0 "$ wy $2=" 0.0 "$ wy $3=" 1.0 "$ p1 $="-0.005987 "$ p2=" $-0.007682 "$ p $3="-0.000000 " />$ $<$ VirtualSite type="localCoords" index="13" atom1="0" atom2="1" atom3="2" wo1 $=" 1.0 "$ wo2 $=" 0.0 "$ wo3 $=" 0.0 "$ wx $1="-1.0 "$ wx $2=" 1.0 "$ wx $3=" 0.0 "$ wy $1="-1.0 "$ wy2="0.0" wy3="1.0" p1=" $0.000000 " \mathrm{p} 2="-0.000000 " \mathrm{p} 3="-0.009591 " />$ $<$ VirtualSite type="localCoords" index="14" atom1="0" atom2="1" atom3="2" wo1 $=" 1.0 "$ wo $2=" 0.0 "$ wo $3=" 0.0 " \mathrm{wx} 1="-1.0 " \mathrm{wx} 2=" 1.0 " \mathrm{wx} 3=" 0.0 " \mathrm{wy} 1="-1.0 "$ wy2="0.0" wy3="1.0" p1="-0.005987" p2=" $-0.007682 " \mathrm{p} 3="-0.000000 " />$ $<!--$ H2-atom charges and positions $-->$

$<$ VirtualSite type="localCoords" index="15" atom $1=" 0 "$ atom $2=" 1$ " atom $3=" 2 "$ wo1 $=" 0.0 "$ wo $2=" 0.0 "$ wo $3=" 1.0 "$ wx $1="-1.0 "$ wx $2=" 0.0 "$ wx $3=" 1.0 "$ wy $1=" 1.0 "$ wy 2 $="-1.0 "$ wy3 $=" 0.0 " \mathrm{p} 1="-0.000905 " \mathrm{p} 2="-0.009551 " \mathrm{p} 3="-0.000000 " />$

$<$ VirtualSite type="localCoords" index="16" atom1="0" atom2="1" atom3="2" wo1 $=" 0.0 "$ wo $2=" 0.0 "$ wo3 $3=1.0 "$ wx $1="-1.0 "$ wx $2=" 0.0 "$ wx $3=" 1.0 "$ wy $1=" 1.0 "$ wy 2 $="-1.0 "$ wy3 $3=0.0 " \mathrm{p} 1="-0.000000 " \mathrm{p} 2="-0.000000 " \mathrm{p} 3="-0.009591 " />$

$<$ VirtualSite type="localCoords" index="17" atom $1=" 0 "$ atom $2=" 1$ " atom $3=" 2 "$ wo1 $=" 0.0 "$ wo $2=" 0.0 "$ wo3 $3=1.0 "$ wx $1="-1.0 "$ wx $2=" 0.0 "$ wx $3=" 1.0 "$ wy $1=" 1.0 "$ wy 2 $="-1.0 "$ wy3 $=" 0.0 " \mathrm{p} 1="-0.000905 " \mathrm{p} 2="-0.009551 " \mathrm{p} 3="-0.000000 " />$

$<$ VirtualSite type="localCoords" index="18" atom $1=" 0 "$ atom $2=" 1 "$ atom $3=" 2 "$ wo1 $=" 0.0 "$ wo $2=" 0.0 "$ wo3 $3=1.0 "$ wx $1="-1.0 "$ wx $2=" 0.0 "$ wx $3=" 1.0 "$ wy $1=" 1.0 "$ wy 2 $="-1.0 "$ wy3 $=" 0.0 " \mathrm{p} 1="-0.009959 " \mathrm{p} 2="-0.000868 " \mathrm{p} 3="-0.000000 " />$

$<$ VirtualSite type="localCoords" index="19" atom $1=" 0 "$ atom $2=" 1$ " atom $3=$ "2" wo1 $=" 0.0 "$ wo $2=" 0.0 "$ wo $3=" 1.0 "$ wx $1="-1.0 "$ wx $2=" 0.0 "$ wx $3=" 1.0 "$ wy $1=" 1.0 "$ wy 2 


$$
="-1.0 " \text { wy3 }=" 0.0 " \mathrm{p} 1="-0.000000 " \mathrm{p} 2="-0.000000 " \mathrm{p} 3="-0.009591 " />
$$

$<$ VirtualSite type="localCoords" index="20" atom $1=" 0 "$ atom $2=" 1$ " atom $3=$ "2" wo1 $=" 0.0 "$ wo $2=" 0.0 "$ wo $3=" 1.0 "$ wx $1="-1.0 "$ wx $2=" 0.0 "$ wx $3=" 1.0 "$ wy $1=" 1.0 "$ wy 2 $="-1.0 "$ wy3 $=" 0.0 " \mathrm{p} 1="\llcorner 0.009959 " \mathrm{p} 2="\llcorner 0.000868 " \mathrm{p} 3="-0.000000 " />$

$</$ Residue $>$

$</$ Residues $>$

$<$ AmoebaBondForce bond -cubic $="-25.5 "$ bond-quartic $=" 379.3125 ">$

$<$ Bond class $1=" 73 "$ class $2=" 74 "$ length="9.584047e-02" $\mathrm{k}=" 2.3331232 \mathrm{e}+05 " />$ $</$ AmoebaBondForce $>$

$<$ AmoebaAngleForce angle - cubic $="-0.014 "$ angle - quartic $=" 5.6 e-05 "$ angle - pentic $="$ $-7 \mathrm{e}-07 "$ angle - sextic $=" 2.2 \mathrm{e}-08 ">$

$<$ Angle class1="74" class2="73" class3="74" k="6.359379296918e-02" angle1=" $1.064826 \mathrm{e}+02 " />$

$</$ AmoebaAngleForce $>$

$<$ AmoebaOutOfPlaneBendForce type="ALLINGER" opbend - cubic $="-0.014$ " opbend - quartic $=" 5.6 \mathrm{e}-05 "$ opbend - pentic $="-7 \mathrm{e}-07 "$ opbend - sextic $=" 2.2 \mathrm{e}-08 ">$ $<!--L P W:$ Mark's $\_$force $\_$field $\_$parsing $\_$code $\_$requires $\_$AmoebaOutOfPlaneBendForce

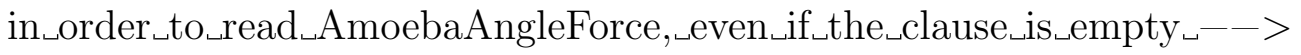

$\_/$AmoebaOutOfPlaneBendForce $>$

$\backsim<$ AmoebaVdwForce $\_$type="BUFFERED-14-7" radiusrule="CUBIC-MEAN" radiustype $=" \mathrm{R}-\mathrm{MIN} "\left\llcorner\right.$ radiussize $="$ DIAMETER" $\_$epsilonrule $=" H H G "\llcorner\mathrm{vdw}-13-$ scale $=" 0.0 "{ }_{\lrcorner} \mathrm{vdw}-14-\mathrm{scale}=" 1.0 "{ }^{\circ} \mathrm{vdw}-15-\mathrm{scale}=" 1.0 ">$

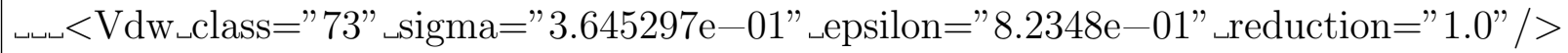

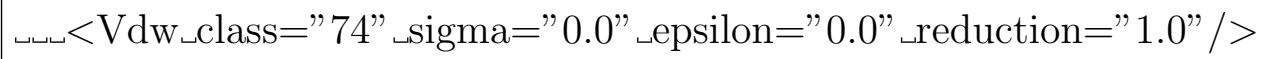
$\sim\left\llcorner\sim \mathrm{Vdw} \_\right.$class $\left.=" 75 "\right\lrcorner \operatorname{sigma}=" 0.0 " \_$epsilon="0.0" reduction="1.0" $/>$

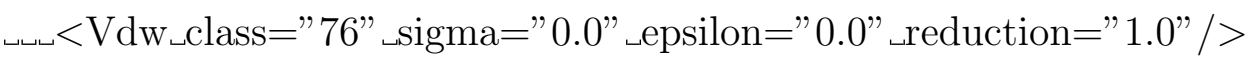

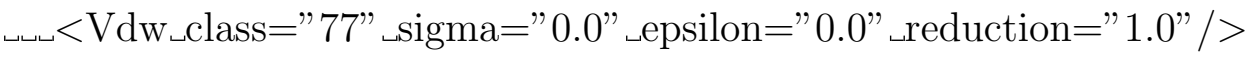




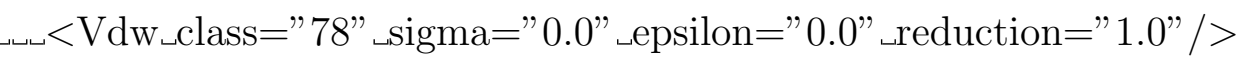

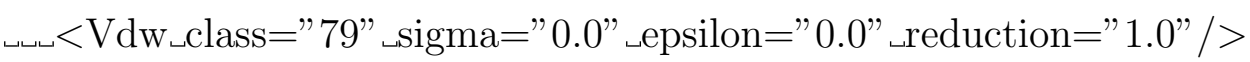

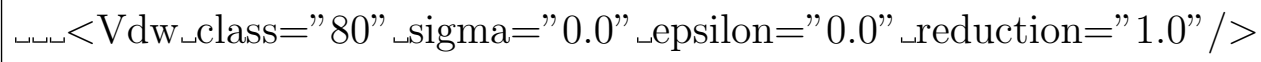

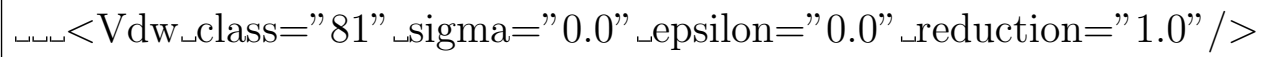

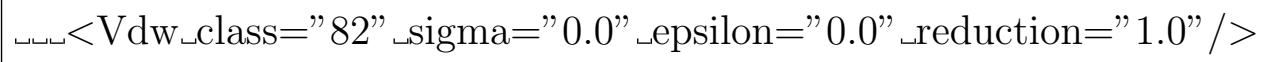

_c_<Vdw_class=" 83" _sigma="0.0"_epsilon="0.0"_reduction="1.0" $/>$

\lrcorner$<\mathrm{Vdw} \_$class $=" 84 "$ "sigma $=" 0.0 "\left\llcorner\right.$ epsilon="0.0" $\_$reduction="1.0" / $>$

$\sim \mathrm{Vdw} \_$class $=" 85 "$ sigma="0.0" $\_$epsilon="0.0" reduction=" $1.0 " />$

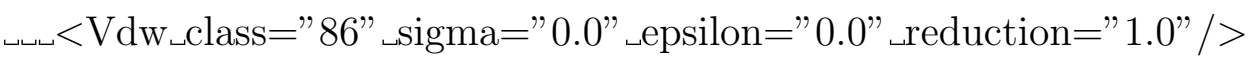

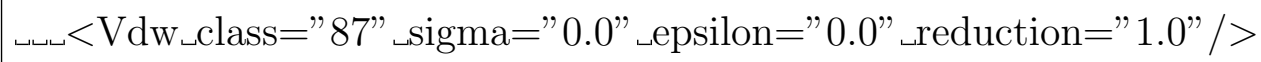

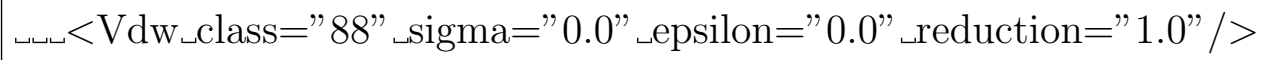

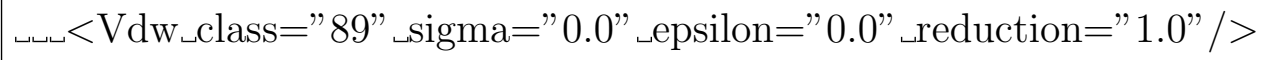

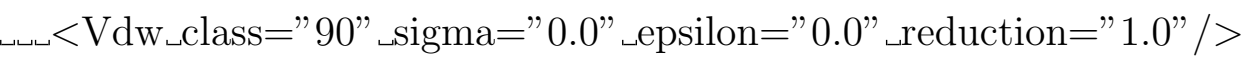

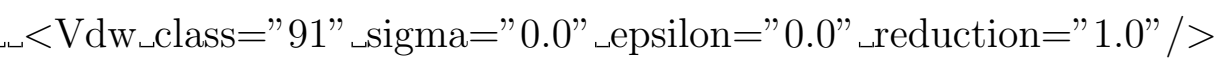

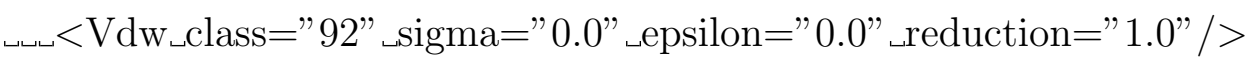

$-</$ AmoebaVdwForce $>$

$\left\llcorner<\right.$ AmoebaMultipoleForce $\_$direct11Scale="0.0" $\lrcorner$direct12Scale="1.0" $\operatorname{direct13Scale="1.0"}$ direct14Scale="1.0" mpole12Scale="0.0" mpole13Scale="0.0" mpole14Scale="0.4"

mpole15Scale="0.8" ـmutual11Scale="1.0" _mutual12Scale="1.0"

$=" 1.0 "\lrcorner$ mutual14Scale="1.0" $\lrcorner$ polar12Scale="0.0" $\lrcorner$ polar13Scale="0.0" $\lrcorner$ polar14Intra

$=" 0.5 "$ polar14Scale $=" 1.0 "$ polar15Scale $=" 1.0 ">$

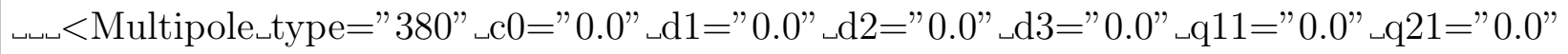
$\llcorner\mathrm{q} 22=" 0.0 "\llcorner\mathrm{q} 31=" 0.0 "\llcorner\mathrm{q} 32=" 0.0 "\lrcorner \mathrm{q} 33=" 0.0 " />$

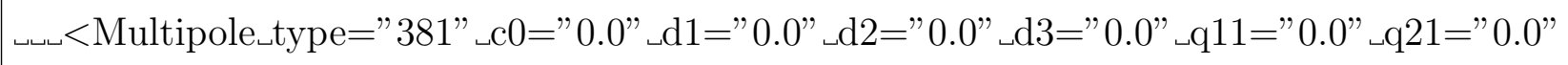
$\llcorner\mathrm{q} 22=" 0.0 "\lrcorner \mathrm{q} 31=" 0.0 "\lrcorner \mathrm{q} 32=" 0.0 "\lrcorner \mathrm{q} 33=" 0.0 " />$ $\sim$ Multipole $\_$type $\left.\left.\left.\left.\left.=" 382 "\right\lrcorner c 0=" 1.613482 "\right\lrcorner \mathrm{d} 1=" 0.0 "\right\lrcorner \mathrm{d} 2=" 0.0 "\right\lrcorner \mathrm{d} 3=" 0.0 "\right\lrcorner \mathrm{q} 11=" 0.0 "\llcorner\mathrm{q} 21$ $=" 0.0 "\llcorner\mathrm{q} 22=" 0.0 "\llcorner\mathrm{q} 31=" 0.0 "\llcorner\mathrm{q} 32=" 0.0 "\llcorner\mathrm{q} 33=" 0.0 " />$ 


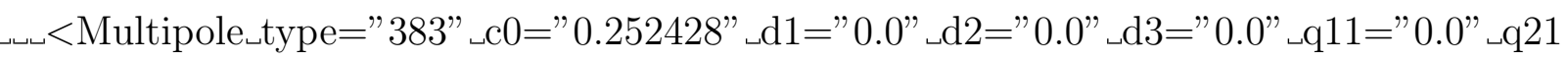
$=" 0.0 "\lrcorner q 22=" 0.0 "\left\llcorner q 31=" 0.0 " \_q 32=" 0.0 "\right\lrcorner q 33=" 0.0 " />$

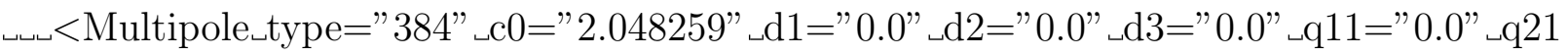
$=" 0.0 "\llcorner q 22=" 0.0 "\llcorner\mathrm{q} 31=" 0.0 "\llcorner\mathrm{q} 32=" 0.0 "\lrcorner \mathrm{q} 33=" 0.0 " />$

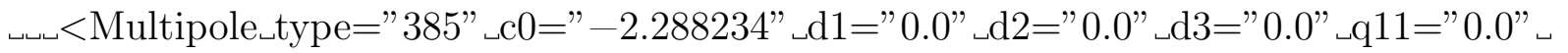
$\mathrm{q} 21=" 0.0 "$ _q $22=" 0.0 " \_\mathrm{q} 31=" 0.0 " \_\mathrm{q} 32=" 0.0 "$-q $33=" 0.0 " />$

$<$ Multipole $\lrcorner$ type $=" 386 "\lrcorner \mathrm{c} 0=" 0.252428 "\lrcorner \mathrm{d} 1=" 0.0 "\lrcorner \mathrm{d} 2=" 0.0 "\lrcorner \mathrm{d} 3=" 0.0 "\lrcorner \mathrm{q} 11=" 0.0 "\llcorner\mathrm{q} 21$ $=" 0.0 "\llcorner q 22=" 0.0 "\llcorner\mathrm{q} 31=" 0.0 "\llcorner\mathrm{q} 32=" 0.0 "\llcorner\mathrm{q} 33=" 0.0 " />$ $<$ Multipole $\lrcorner$ type $=" 387 "\lrcorner c 0="-1.581353 "\lrcorner \mathrm{d} 1=" 0.0 "\llcorner\mathrm{~d} 2=" 0.0 "\lrcorner \mathrm{d} 3=" 0.0 "\llcorner\mathrm{q} 11=" 0.0 "$ 。 $\mathrm{q} 21=" 0.0 "\llcorner\mathrm{q} 22=" 0.0 "\llcorner\mathrm{q} 31=" 0.0 "\llcorner\mathrm{q} 32=" 0.0 "\llcorner\mathrm{q} 33=" 0.0 " />$

$\sim \sim<$ Multipole type $=" 388 "\lrcorner \mathrm{c} 0=" 2.012216 "\lrcorner \mathrm{d} 1=" 0.0 "\lrcorner \mathrm{d} 2=" 0.0 "\lrcorner \mathrm{d} 3=" 0.0 "\lrcorner \mathrm{q} 11=" 0.0 "\lrcorner \mathrm{q} 21$ $=" 0.0 "\llcorner q 22=" 0.0 "\llcorner\mathrm{q} 31=" 0.0 "\llcorner\mathrm{q} 32=" 0.0 "\llcorner\mathrm{q} 33=" 0.0 " />$

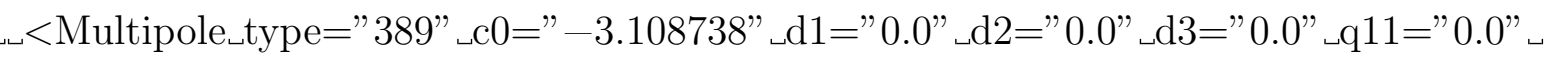
$\mathrm{q} 21=" 0.0 "\llcorner\mathrm{q} 22=" 0.0 "\llcorner\mathrm{q} 31=" 0.0 "\llcorner\mathrm{q} 32=" 0.0 "\llcorner\mathrm{q} 33=" 0.0 " />$ $<$ Multipole $\lrcorner$ type $=" 390 "\lrcorner \mathrm{c} 0=" 2.012216 "\lrcorner \mathrm{d} 1=" 0.0 "\lrcorner \mathrm{d} 2=" 0.0 "\lrcorner \mathrm{d} 3=" 0.0 "\lrcorner \mathrm{q} 11=" 0.0 "\llcorner\mathrm{q} 21$ $=" 0.0 "\llcorner\mathrm{q} 22=" 0.0 "\llcorner\mathrm{q} 31=" 0.0 "\llcorner\mathrm{q} 32=" 0.0 "\llcorner\mathrm{q} 33=" 0.0 " />$

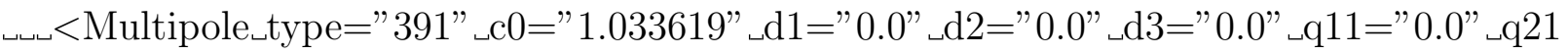
$=" 0.0 "\llcorner q 22=" 0.0 "\llcorner q 31=" 0.0 "\llcorner q 32=" 0.0 "\llcorner q 33=" 0.0 " />$

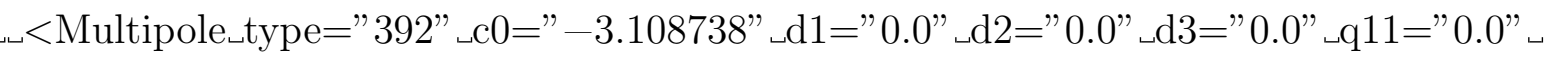
$\mathrm{q} 21=" 0.0 "\lrcorner \mathrm{q} 22=" 0.0 "\llcorner\mathrm{q} 31=" 0.0 "\llcorner\mathrm{q} 32=" 0.0 "\lrcorner \mathrm{q} 33=" 0.0 " />$

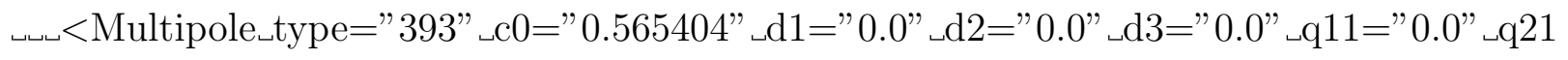
$=" 0.0 "\llcorner q 22=" 0.0 "\llcorner q 31=" 0.0 "\llcorner q 32=" 0.0 "\lrcorner q 33=" 0.0 " />$

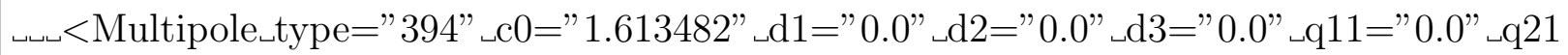
$=" 0.0 "\llcorner q 22=" 0.0 "\llcorner q 31=" 0.0 "\llcorner q 32=" 0.0 "\lrcorner q 33=" 0.0 " />$ $\sim$ Multipole $\lrcorner$ type $=" 395 "\lrcorner c 0=" 0.252428 "\lrcorner \mathrm{d} 1=" 0.0 "\lrcorner \mathrm{d} 2=" 0.0 "\lrcorner \mathrm{d} 3=" 0.0 "\lrcorner \mathrm{q} 11=" 0.0 "\lrcorner \mathrm{q} 21$ $=" 0.0 "\llcorner q 22=" 0.0 "\llcorner q 31=" 0.0 "\llcorner q 32=" 0.0 "\llcorner q 33=" 0.0 " />$ $<$ Multipole $\lrcorner$ type $=" 396 "\llcorner\mathrm{c} 0=" 2.048259 "\lrcorner \mathrm{d} 1=" 0.0 "\lrcorner \mathrm{d} 2=" 0.0 "\llcorner\mathrm{~d} 3=" 0.0 "\llcorner\mathrm{q} 11=" 0.0 "\llcorner\mathrm{q} 21$ 


$$
=" 0.0 "\llcorner q 22=" 0.0 "\llcorner\mathrm{q} 31=" 0.0 "\llcorner\mathrm{q} 32=" 0.0 "\llcorner\mathrm{q} 33=" 0.0 " />
$$

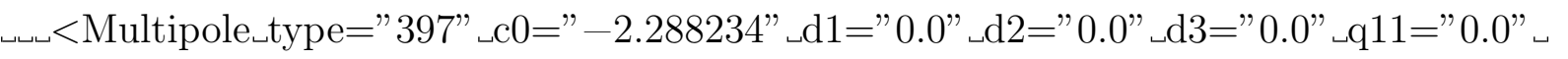

$$
\mathrm{q} 21=" 0.0 "\llcorner\mathrm{q} 22=" 0.0 "\llcorner\mathrm{q} 31=" 0.0 "\llcorner\mathrm{q} 32=" 0.0 "\llcorner\mathrm{q} 33=" 0.0 " />
$$

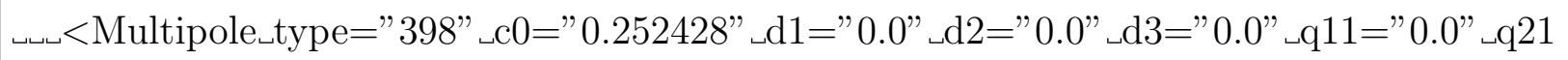

$$
=" 0.0 "-q 22=" 0.0 "\llcorner\mathrm{q} 31=" 0.0 "-\mathrm{q} 32=" 0.0 "-\mathrm{q} 33=" 0.0 " />
$$

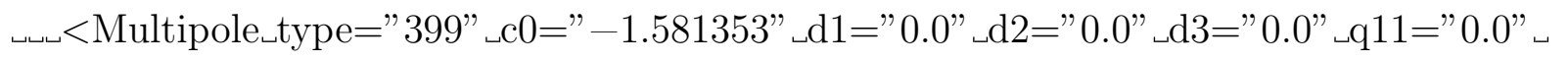
$\mathrm{q} 21=" 0.0 " \sqcup \mathrm{q} 22=" 0.0 "$ q $31=" 0.0 "\llcorner\mathrm{q} 32=" 0.0 "\llcorner\mathrm{q} 33=" 0.0 " />$

$\llcorner$ Polarize $\lrcorner$ type $=" 381 "$ ppolarizability $=" 5.048434386104 \mathrm{e}-04 "$.thole $=" 2.36164 \mathrm{e}-03 "$

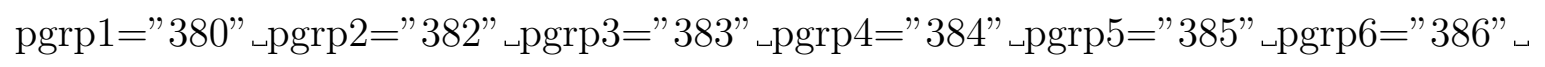

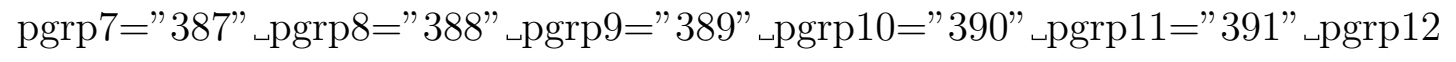

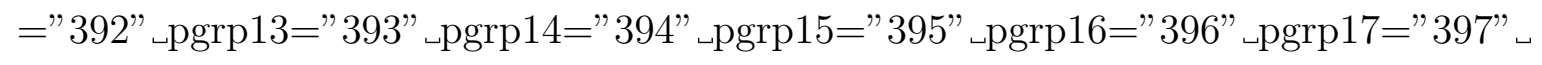
pgrp18="398" $\lrcorner \operatorname{pgrp} 19=" 399 " />$ $\sim_{\llcorner}<$Polarize $\lrcorner$type $=" 382 "$ polarizability $=" 5.048434386104 \mathrm{e}-08 "$ "thole $=" 2.36164 \mathrm{e}-03 "$

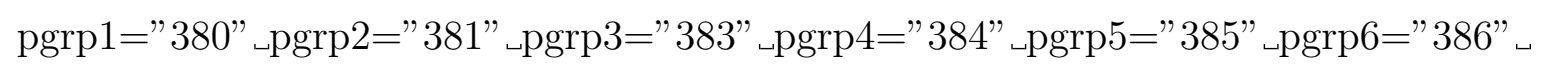

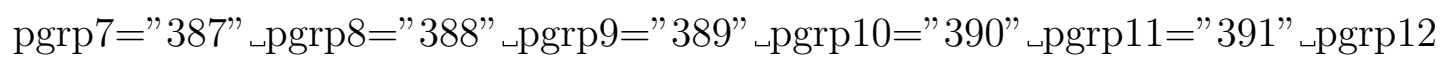

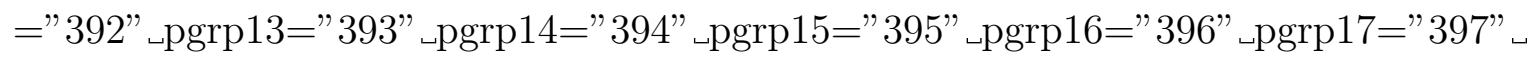
pgrp18="398" $\lrcorner \operatorname{pgrp} 19=" 399 " />$

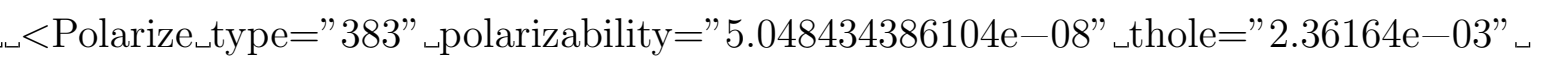

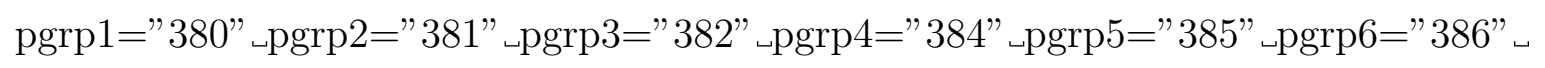

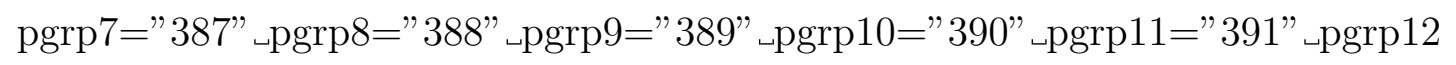

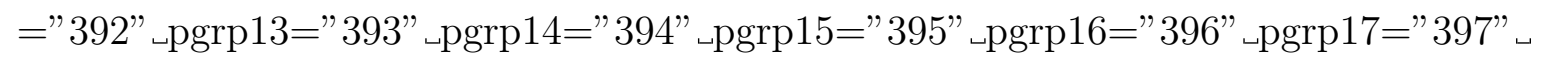
pgrp18="398" $\lrcorner \operatorname{pgrp} 19=" 399 " />$

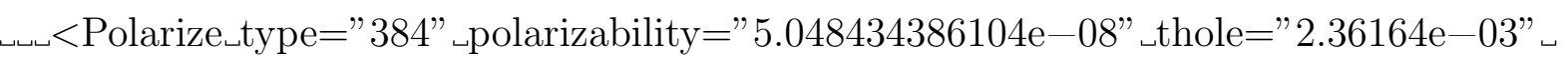

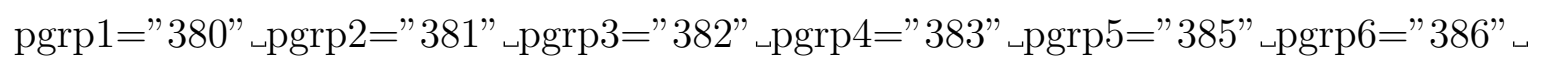

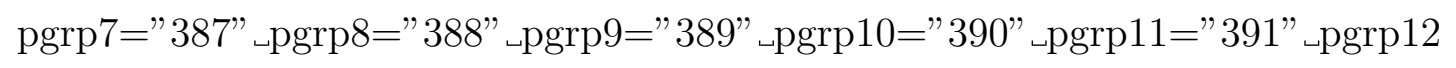

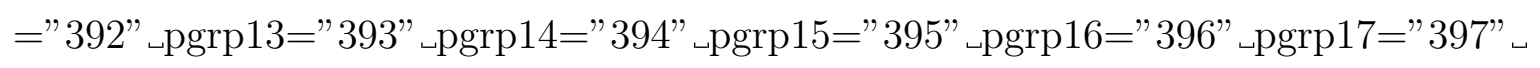
pgrp18="398" $\lrcorner \operatorname{pgrp} 19=" 399 " />$ 


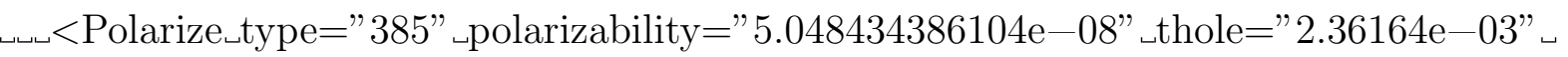

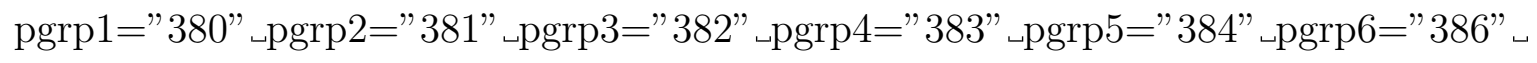

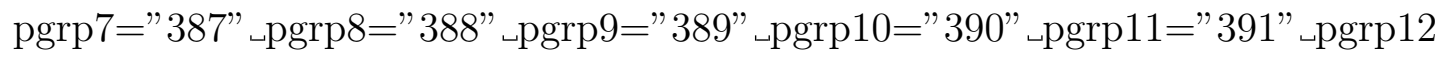

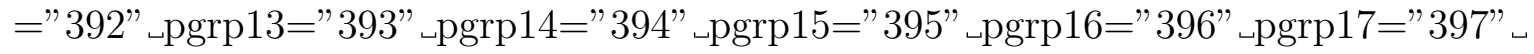
pgrp18="398" -pgrp19="399" /> $<$ Polarize $\_$type $=" 386 "$ "polarizability $=" 5.048434386104 \mathrm{e}-08 "$ „thole $=" 2.36164 \mathrm{e}-03 "$

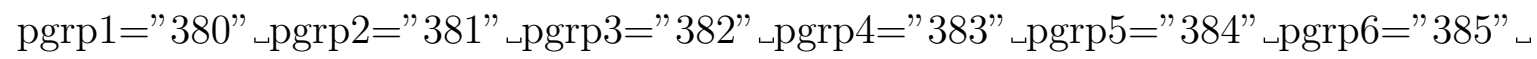

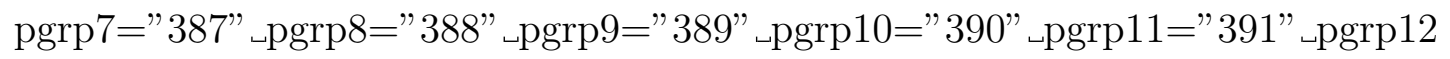

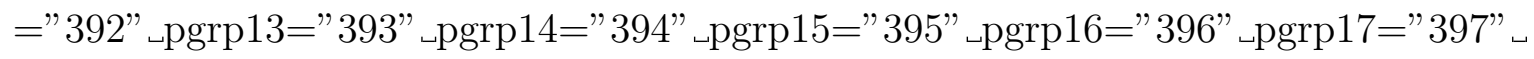
pgrp18="398" -pgrp19="399"/>

$\llcorner\sim<$ Polarize $\lrcorner$ type $=" 387 "$ "polarizability $=" 5.048434386104 \mathrm{e}-08$ " thole $=" 2.36164 \mathrm{e}-03 "$

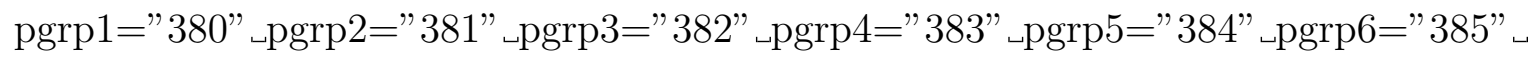

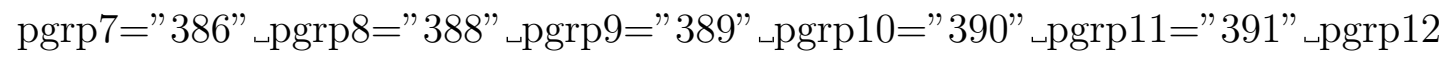

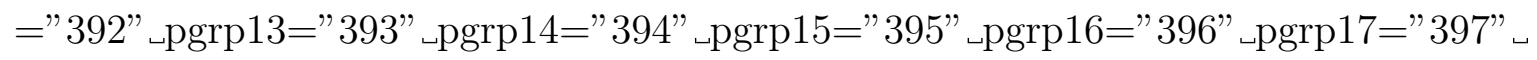
pgrp18="398" $\lrcorner \operatorname{pgrp} 19=" 399 " />$

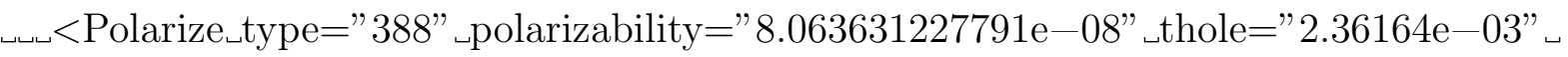

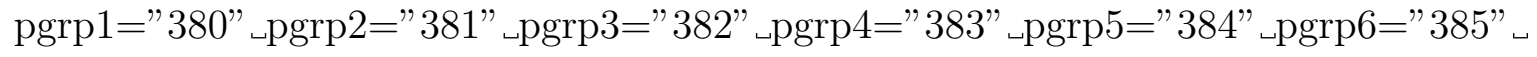

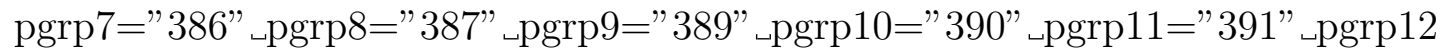

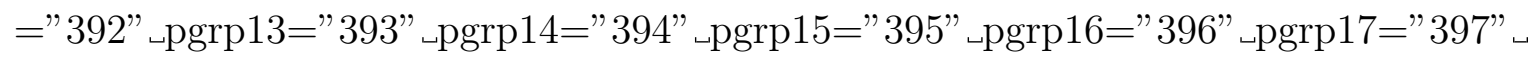
pgrp18="398" $\lrcorner \operatorname{pgrp} 19=" 399 " />$

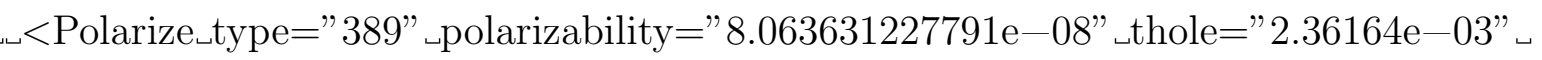

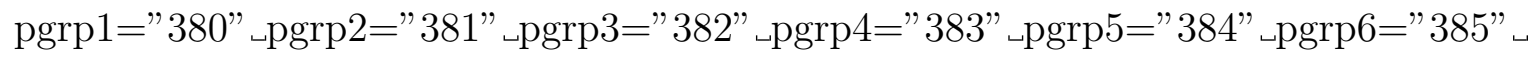

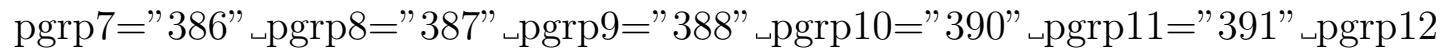

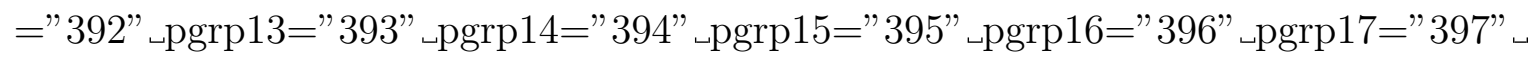
pgrp18="398" $\lrcorner \operatorname{pgrp} 19=" 399 " />$ $<$ Polarize $\_$type $=" 390 "$ polarizability $=" 8.063631227791 \mathrm{e}-08 "$.thole $=" 2.36164 \mathrm{e}-03 "$

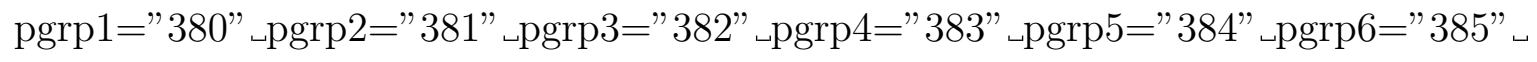




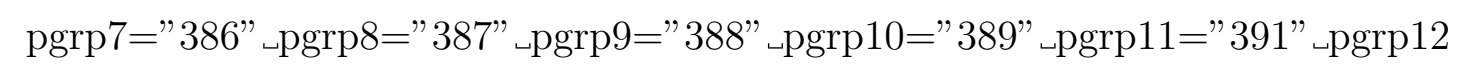

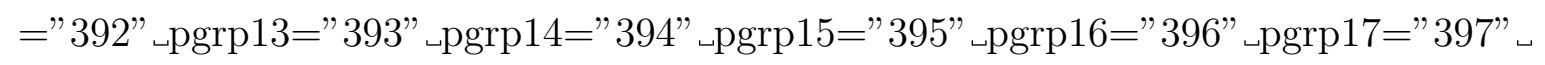$$
\text { pgrp18="398" }\lrcorner \operatorname{pgrp} 19=" 399 " />
$$

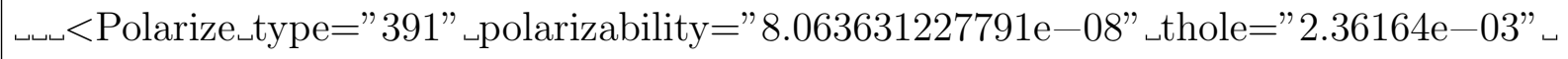

$$
\text { pgrp1="380" „pgrp2="381" -pgrp3="382" -pgrp4="383" -pgrp5="384" -pgrp6=" 385" }
$$

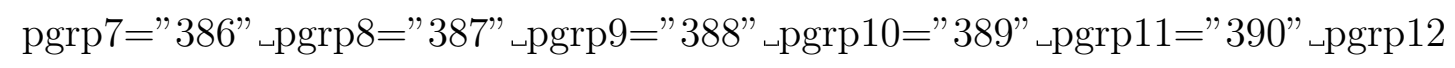

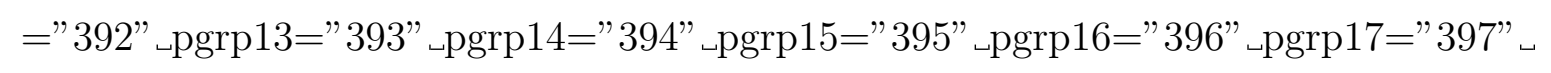$$
\text { pgrp18="398" } \operatorname{pgrp} 19=" 399 " />
$$

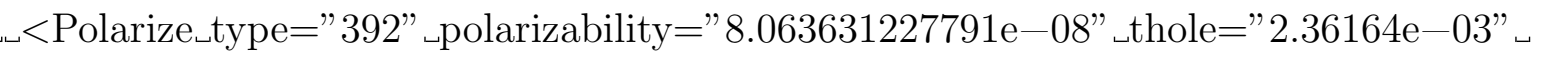

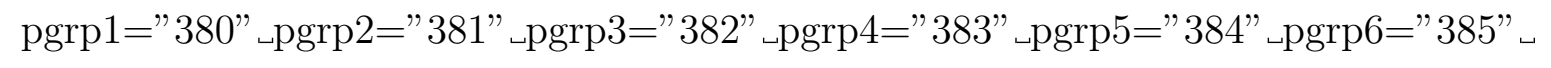

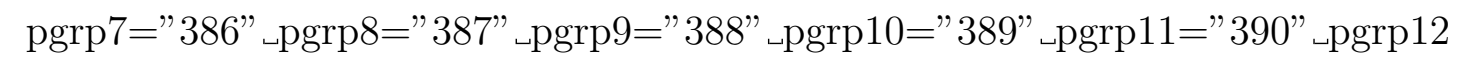

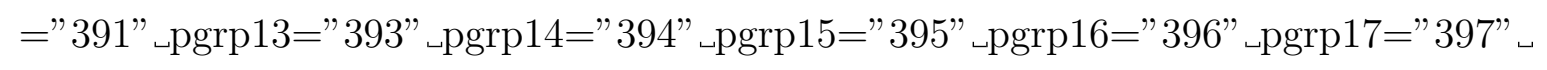$$
\operatorname{pgrp} 18=" 398 " \sqcup \operatorname{pgrp} 19=" 399 " />
$$

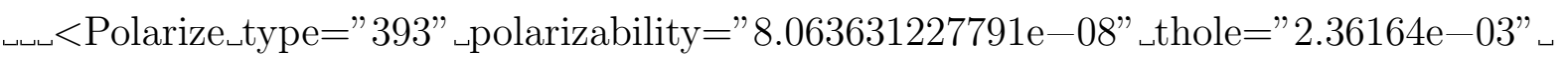

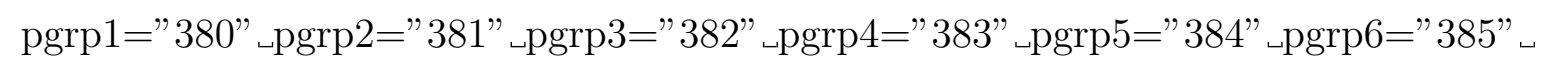

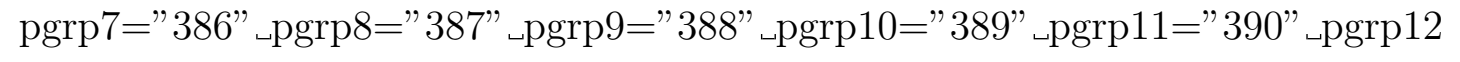

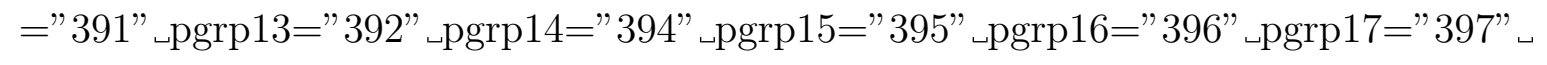
$\operatorname{pgrp} 18=" 398 " \sqcup \operatorname{pgrp} 19=" 399 " />$

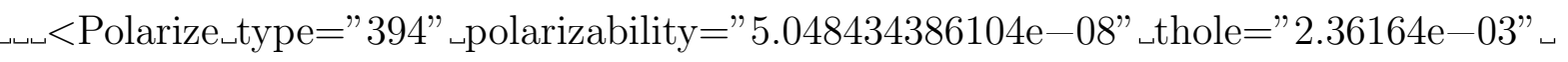

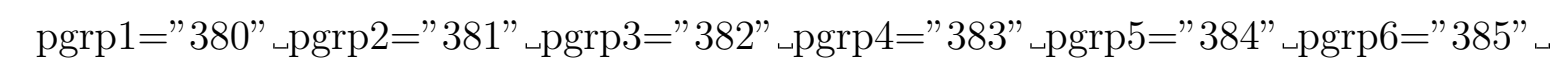

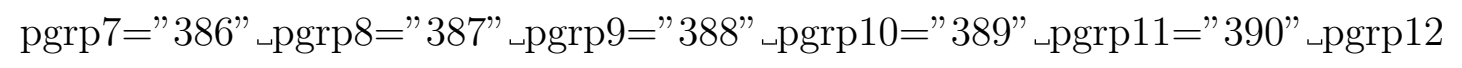

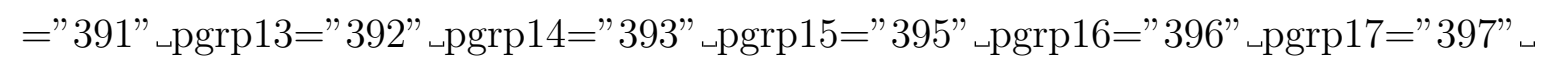
pgrp18="398" $-\operatorname{pgrp} 19=" 399 " />$

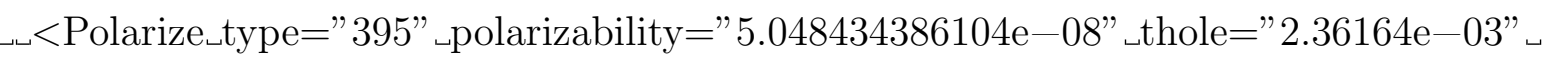

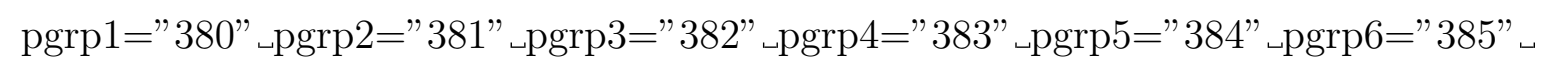

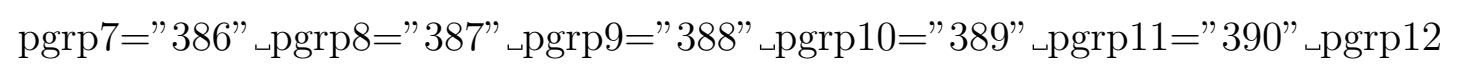

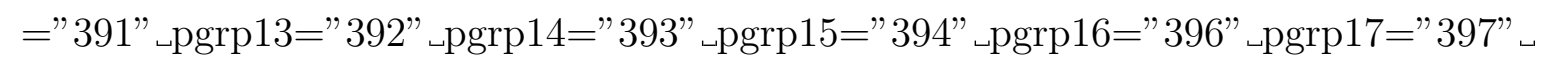




$$
\text { pgrp18="398" „pgrp19="399"/> }
$$

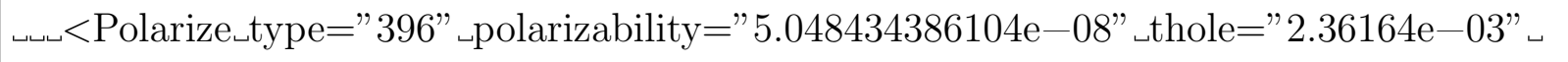

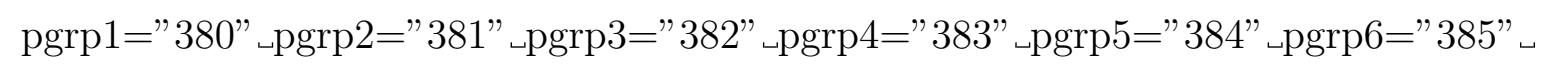

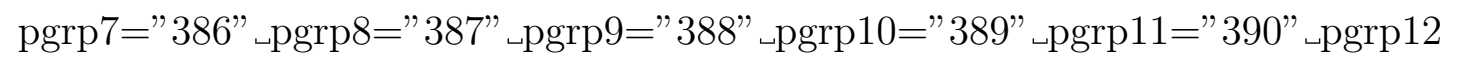

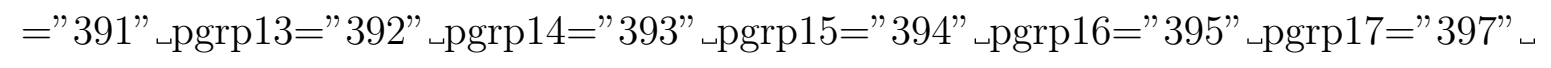
$\operatorname{pgrp} 18=" 398 "-p g r p 19=" 399 " />$ $\llcorner\llcorner<$ Polarize $\lrcorner$ type $=" 397 "$ "polarizability =" 5.048434386104e-08" .thole="2.36164e-03"

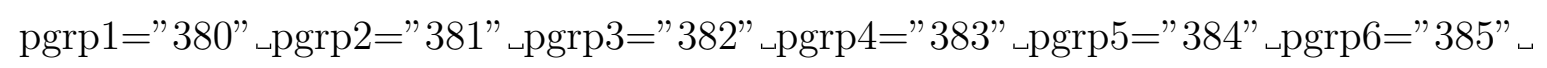

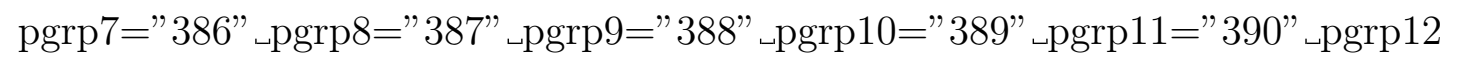

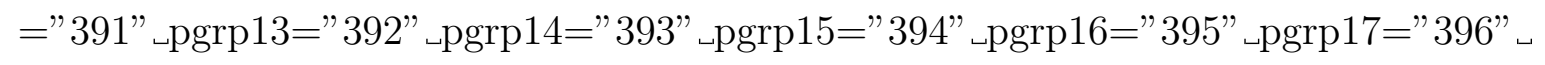
pgrp18="398" $\lrcorner \operatorname{pgrp} 19=" 399 " />$

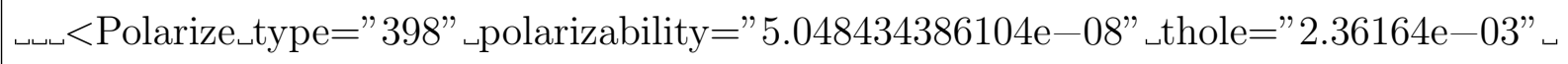

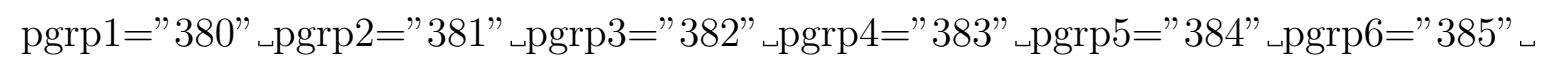

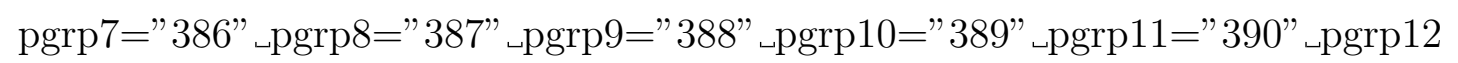

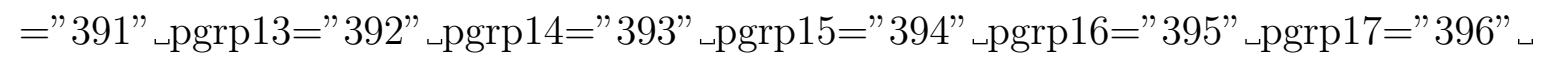
pgrp18="397" $\lrcorner \operatorname{pgrp} 19=" 399 " />$

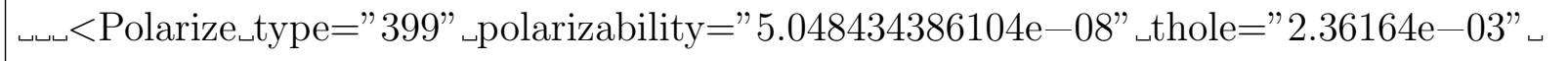

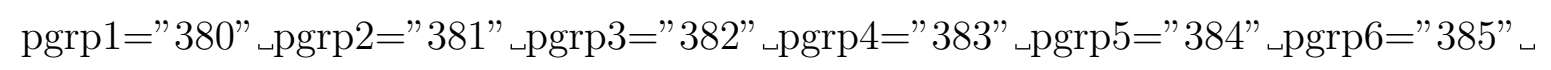

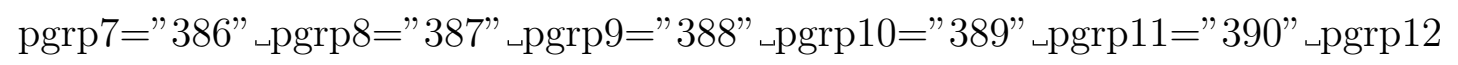

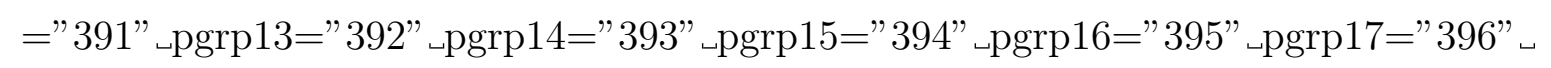
pgrp18="397" $\lrcorner \operatorname{pgrp} 19=" 398 " />$

$\leftarrow</$ AmoebaMultipoleForce $>$

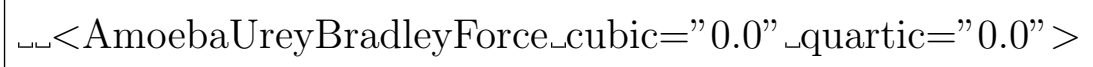

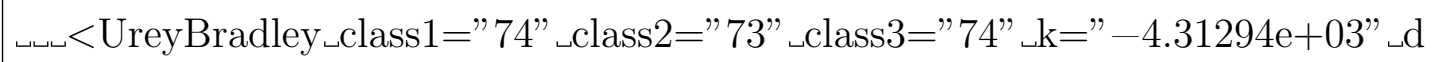

$$
=" 1.535676676685 \mathrm{e}-01 " />
$$

$\_$</AmoebaUreyBradleyForce $>$

$</$ ForceField $>$ 


\section{Error Reduction in MDCMs}

In the following it is demonstrated that applying constraints to charge magnitudes during MDCM fitting can reduce the corresponding error in electrostatic interaction energy between molecules of arbitrary types and relative spatial orientations.

The MDCM optimal fitted solution without applied constraints minimizes errors in the MEP, rather than the electrostatic interaction energy relevant to MD simulations. Fitting to the electrostatic interaction energy directly would avoid this issue, but also lead to biased sampling of the electric field around the molecule during fitting by focusing on those regions relevant to the electrostatic interactions included in the finite training set. It is more difficult to generate sufficiently large, heterogeneous training sets of reference electrostatic interaction energies between non-polarized monomers than to generate the more straightforward MEP across a spatial grid. A good solution is therefore to solve the more straightforward MEP fitting problem with constraints introduced to ensure that the resulting charge models will also perform well for the closely related electrostatic interaction energy between molecules.

To relate the error in the MEP to the resulting error in the electrostatic interaction between molecules, we start with a description of the residual error in the MEP of an MDCM for molecule 'a' after fitting:

$$
V_{\mathrm{a}}(\mathbf{r})=\int_{\Omega_{\mathrm{a}}} \frac{\rho_{\mathrm{a}}\left(\mathbf{r}_{\mathbf{a}}\right)}{\left|\mathbf{r}-\mathbf{r}_{\mathbf{a}}\right|} d r_{\mathrm{a}}=\sum_{n=1}^{n_{q, \mathrm{a}}} \frac{q_{\mathrm{a}, n}}{\left|\mathbf{r}-\mathbf{r}_{\mathbf{a}, n}\right|}+\delta V_{\mathrm{a}}(\mathbf{r})
$$

for $\mathbf{r}$ sampled outside the molecular surface used for fitting. $\rho_{\mathrm{a}}\left(\mathbf{r}_{\mathbf{a}}\right)$ is the reference electron density of molecule ' $\mathrm{a}$ ' at point $\mathbf{r}_{\mathbf{a}}$ in the molecular volume $\Omega_{a} . q_{\mathrm{a}, n}$ is charge $n$ of the fitted MDCM model with $\mathbf{r}_{\mathbf{a}, n}$ the position of the charge, and $\delta V_{\mathrm{a}}(\mathbf{r})$ is the residual error in the MEP after fitting the MDCM.

This can be related to the error in interaction with a single external charge, $q_{\mathrm{b}}$ at $\mathbf{r}_{\mathbf{b}}$ :

$$
U_{\mathrm{ab}}=q_{\mathrm{b}} \int_{\Omega_{\mathrm{a}}} \frac{\rho_{\mathrm{a}}\left(\mathbf{r}_{\mathbf{a}}\right)}{\left|\mathbf{r}_{\mathbf{b}}-\mathbf{r}_{\mathbf{a}}\right|} d r_{\mathrm{a}}=q_{\mathrm{b}} \sum_{n=1}^{n_{q, \mathrm{a}}} \frac{q_{\mathrm{a}, n}}{\left|\mathbf{r}_{\mathbf{b}}-\mathbf{r}_{\mathbf{a}, n}\right|}+q_{\mathrm{b}} \delta V_{\mathrm{a}}\left(\mathbf{r}_{\mathbf{b}}\right)
$$


Using the exact interaction between two molecules, a double integral over the molecular volumes of $a$ and $b$ :

$$
U_{\mathrm{ab}}=\int_{\Omega_{\mathrm{a}}} \int_{\Omega_{\mathrm{b}}} \frac{\rho_{\mathrm{a}}\left(\mathbf{r}_{\mathbf{a}}\right) \rho_{\mathrm{b}}\left(\mathbf{r}_{\mathbf{b}}\right)}{\left|\mathbf{r}_{\mathbf{b}}-\mathbf{r}_{\mathbf{a}}\right|} d r_{\mathrm{a}} d r_{\mathrm{b}}
$$

and inserting (12) and rearranging yields

$$
U_{\mathrm{ab}}=\sum_{n=1}^{\mathrm{n}_{\mathrm{q}, \mathrm{a}}} q_{\mathrm{a}, n} \int_{\Omega_{\mathrm{b}}} \frac{\rho_{\mathrm{b}}\left(\mathbf{r}_{\mathbf{b}}\right)}{\left|\mathbf{r}_{\mathbf{b}}-\mathbf{r}_{\mathbf{a}, n}\right|}+\int_{\Omega_{\mathrm{b}}} \rho_{\mathrm{b}}\left(\mathbf{r}_{\mathbf{b}}\right) \delta V_{\mathrm{a}}\left(\mathbf{r}_{\mathbf{b}}\right) d r_{\mathrm{b}}
$$

Using (12) again for molecule $b$ :

$$
U_{\mathrm{ab}}=\sum_{n=1}^{\mathrm{n}_{\mathrm{q}, \mathrm{a}}} \sum_{m=1}^{\mathrm{n}_{\mathrm{q}, \mathrm{b}}} \frac{q_{\mathrm{a}, n} q_{\mathrm{b}, m}}{\left|\mathbf{r}_{\mathbf{b}, m}-\mathbf{r}_{\mathbf{a}, n}\right|}+\sum_{n=1}^{n_{q, \mathrm{a}}} q_{\mathrm{a}, n} \delta V_{\mathrm{b}}\left(\mathbf{r}_{\mathbf{a}, n}\right)+\int_{\Omega_{\mathrm{b}}} \rho_{\mathrm{b}}\left(\mathbf{r}_{\mathbf{b}}\right) \delta V_{\mathrm{a}}\left(\mathbf{r}_{\mathbf{b}}\right) d r_{\mathrm{b}}
$$

or, equivalently:

$$
U_{\mathrm{ab}}=\sum_{n=1}^{\mathrm{n}_{\mathrm{q}, \mathrm{b}}} \sum_{m=1}^{\mathrm{n}_{\mathrm{q}, \mathrm{a}}} \frac{q_{\mathrm{b}, n} q_{\mathrm{a}, m}}{\left|\mathbf{r}_{\mathbf{a}, m}-\mathbf{r}_{\mathbf{b}, n}\right|}+\sum_{n=1}^{n_{q, \mathrm{~b}}} q_{\mathrm{b}, n} \delta V_{\mathrm{a}}\left(\mathbf{r}_{\mathbf{b}, n}\right)+\int_{\Omega_{a}} \rho_{\mathrm{a}}\left(\mathbf{r}_{\mathbf{a}}\right) \delta V_{\mathrm{b}}\left(\mathbf{r}_{\mathbf{a}}\right) d r_{\mathrm{a}}
$$

Minimizing the $\delta V_{\mathrm{a}}(\mathbf{r})$ error term in (12) requires simply a charge model that describes the MEP as closely as possible to the corresponding Coulomb integral over the molecular charge density. In practice this often results in large fitted charges, of the order of several a.u. The error terms in (16) and (17) for the electrostatic interaction energy, however, show that the error in the MEP is multiplied by the magnitude of each interacting charge. While error cancellation is possible it is not guaranteed, so the presence of larger charges can lead to an amplification of the error in the MEP. For a typical fitting problem it has been observed that for a given MDCM model containing large charges, the error in the electrostatic interaction energy is amplified in some regions but remains small due to error cancellation in others. It is therefore safest to constrain the magnitude of the fitted charges to remain as low as possible while still achieving an acceptable error in the fitted MEP, i.e. to find a compromise solution with low errors in the fitted MEP and the smallest possible charges. 


\section{5 iAMOEBA MDCM OpenMM force field definition file}

The following "XML" format force field definition file defines the iAMOEBA/MDCM implementation for use in OpenMM. Dummy atom types could again potentially be consolidated as all MDCM dummy atoms also share the same mass and VDW, and sites belonging to the same atom-type also have the same polarizability.

$$
\begin{aligned}
& <\text { ForceField }> \\
& <\text { AtomTypes }> \\
& <\text { Type name="380" class="73" element="O" mass="15.999" / > } \\
& <\text { Type name="381" class="74" element="H" mass="1.008" / > } \\
& <\text { Type name="382" class="75" mass="0" } /> \\
& <\text { Type name="383" class }=" 76 " \text { mass }=" 0 " /> \\
& <\text { Type name="384" class="77" mass }=" 0 " /> \\
& <\text { Type name="385" class }=" 78 " \text { mass }=" 0 " /> \\
& <\text { Type name }=" 386 " \text { class }=" 79 " \text { mass }=" 0 " /> \\
& <\text { Type name="387" class }=" 80 " \text { mass }=" 0 " /> \\
& <\text { Type name }=" 388 " \text { class }=" 81 " \text { mass }=" 0 " /> \\
& <\text { Type name="389" class=" 82" mass="0" / > } \\
& <\text { Type name="390" class=" 83" mass="0" / > } \\
& <\text { Type name="391" class="84" mass }=" 0 " /> \\
& </ \text { AtomTypes }> \\
& <\text { Residues }> \\
& <\text { Residue name="HOH" }> \\
& <\text { Atom name="O" type="380" / > } \\
& <\text { Atom name="H1" type="381" / > } \\
& <\text { Atom name="H2" type="381" / > }
\end{aligned}
$$


$<$ Atom name="M1" type="382" / >

$<$ Atom name="M2" type="383" $/>$

$<$ Atom name="M3" type="384" $/>$

$<$ Atom name="M4" type $=" 385 " />$

$<$ Atom name="M5" type $=" 386 " />$

$<$ Atom name="M6" type="387" $/>$

$<$ Atom name="M7" type $=" 388 " />$

$<$ Atom name="M8" type $=" 389 " />$

$<$ Atom name="M9" type $=" 390 " />$

$<$ Atom name="M10" type $=" 391 " />$

$<$ Bond from $=" 0 "$ to $=" 2 " />$

$<$ Bond from=" $0 "$ to $=" 1 " />$

$<!--H 1-$ atom charges and positions - - >

$<$ VirtualSite type="localCoords" index="3" atom1="0" atom2="1" atom3="2" wo1=

"0.0" wo $2=" 1.0$ " wo3=" $0.0 "$ wx $1="-1.0$ " wx $2=" 1.0$ " wx3=" $0.0 "$ wy $1="-1.0 "$ wy 2

$=" 0.0 "$ wy3 $=" 1.0 " \mathrm{p} 1="\llcorner 0.034734 " \mathrm{p} 2="-0.002091 " \mathrm{p} 3="\llcorner 0.000000 " />$

$<$ VirtualSite type="localCoords" index=" 4" atom1="0" atom2="1" atom3="2" wo1= "0.0" wo $2=" 1.0$ " wo3=" $0.0 "$ wx $1="-1.0 "$ wx $2=" 1.0 "$ wx $3=" 0.0 "$ wy $1="-1.0 "$ wy 2 $=" 0.0 "$ wy3 $=" 1.0 " \mathrm{p} 1="-0.016513 " \mathrm{p} 2="-0.004524 " \mathrm{p} 3=" \_0.000000 " />$

$<$ VirtualSite type $="$ localCoords" index $=" 5$ " atom1="0" atom2="1" atom3=" 2 " wo1= " $0.0 "$ wo $2=" 1.0 "$ wo3=" $0.0 "$ wx $1="-1.0 "$ wx $2=" 1.0 "$ wx $3=" 0.0 "$ wy $1="-1.0 "$ wy 2 $=" 0.0 "$ wy3 $=" 1.0 " \mathrm{p} 1="-0.005283 " \mathrm{p} 2="-0.019053 " \mathrm{p} 3="-0.000000 " />$

$<$ VirtualSite type $="$ localCoords" index $=" 6$ " atom1="0" atom2=" 1 " atom3=" 2" wo1= "0.0" wo2 $=" 1.0 "$ wo3=" $0.0 "$ wx $1="-1.0 "$ wx $2=" 1.0 "$ wx $3=" 0.0 "$ wy $1="-1.0 "$ wy 2 $=" 0.0 "$ wy3 $=" 1.0 " \mathrm{p} 1="-0.005651 " \mathrm{p} 2="-0.014961 " \mathrm{p} 3="-0.000189 " />$ $<!--O$-atom charges and positions $-->$ 
$<$ VirtualSite type="localCoords" index $=" 7 "$ atom $1=" 0 "$ atom $2=" 1 "$ atom $3=" 2 "$ wo1= "1.0" wo2=" $0.0 "$ wo3 $=" 0.0 "$ wx $1="-1.0 "$ wx $2=" 1.0 "$ wx $3=" 0.0 "$ wy $1="-1.0 "$ wy 2 $=" 0.0 "$ wy3="1.0" p1=" $-0.002946 " \mathrm{p} 2="-0.010093 " \mathrm{p} 3=" 0.040674 " />$

$<$ VirtualSite type="localCoords" index=" 8" atom1="0" atom $2=" 1 "$ atom $3=" 2 "$ wo1= $" 1.0 "$ wo2 $=" 0.0 "$ wo3 $=" 0.0 "$ wx $1="-1.0 "$ wx $2=" 1.0 "$ wx $3=" 0.0 "$ wy $1="-1.0 "$ wy 2 $=" 0.0 "$ wy3 $=" 1.0 " \mathrm{p} 1="-0.002031 " \mathrm{p} 2="-0.008820 " \mathrm{p} 3="-0.031699 " />$ $<!--$ H2-atom charges and positions $->$

$<$ VirtualSite type="localCoords" index="9" atom $1=" 0 "$ atom $2=" 1 "$ atom $3=" 2 "$ wo1= "0.0" wo $2=" 0.0 "$ wo3 $=" 1.0 "$ wx $1="-1.0 "$ wx $2=" 0.0 "$ wx $3=" 1.0 "$ wy $1=" 1.0 "$ wy $2=$ "-1.0" wy3="0.0" p1=" $0.002756 " \mathrm{p} 2="-0.016593 " \mathrm{p} 3="-0.000082 " />$

$<$ VirtualSite type="localCoords" index="10" atom $1=" 0 "$ atom $2=" 1$ " atom $3=$ "2" wo1 $=" 0.0 "$ wo $2=" 0.0 "$ wo3 $3=" 1.0 "$ wx $1="-1.0 "$ wx $2=" 0.0 "$ wx $3=" 1.0 "$ wy $1=" 1.0 "$ wy 2 $="-1.0 "$ wy3 $=" 0.0 " \mathrm{p} 1="-0.004779 " \mathrm{p} 2="-0.018380 " \mathrm{p} 3="-0.000209 " />$

$<$ VirtualSite type="localCoords" index="11" atom1="0" atom2="1" atom3="2" wo1 $=" 0.0 "$ wo $2=" 0.0 "$ wo3 $3=1.0 "$ wx $1="-1.0 "$ wx $2=" 0.0 "$ wx $3=" 1.0 "$ wy $1=" 1.0 "$ wy 2 $="-1.0 "$ wy3 $=" 0.0 " \mathrm{p} 1="-0.037642 " \mathrm{p} 2="-0.005199 " \mathrm{p} 3="-0.000000 " />$

$<$ VirtualSite type="localCoords" index="12" atom1="0" atom2="1" atom3="2" wo1 $=" 0.0 "$ wo $2=" 0.0 "$ wo3 $3=1.0 "$ wx $1="-1.0 "$ wx $2=" 0.0 "$ wx $3=" 1.0 "$ wy $1=" 1.0 "$ wy 2 $="-1.0 "$ wy3 $=" 0.0 "$ p1 $="-0.020099 " \mathrm{p} 2="-0.003550 " \mathrm{p} 3="-0.000000 " />$

$</$ Residue $>$

$</$ Residues $>$

$<$ AmoebaBondForce bond-cubic=" $-25.5 "$ bond-quartic $=" 379.3125 ">$

$<$ Bond class $1=" 73 "$ class $2=" 74 "$ length="9.584047e-02" k="2.3331232e+05" / > $</$ AmoebaBondForce $>$

$<$ AmoebaAngleForce angle - cubic $="-0.014 "$ angle - quartic $=" 5.6 \mathrm{e}-05 "$ angle - pentic $="$ $-7 \mathrm{e}-07 "$ angle - sextic $=" 2.2 \mathrm{e}-08 ">$

$<$ Angle class $1=" 74 "$ class2="73" class3="74" $\mathrm{k}=" 6.359379296918 \mathrm{e}-02 "$ angle1=" 


\section{$1.064826 \mathrm{e}+02 " />$}

$</$ AmoebaAngleForce $>$

$<$ AmoebaOutOfPlaneBendForce type="ALLINGER" opbend - cubic=" -0.014 " opbend

- quartic $=" 5.6 \mathrm{e}-05 "$ opbend - pentic $="-7 \mathrm{e}-07$ " opbend - sextic $=" 2.2 \mathrm{e}-08 ">$

$<!--L P W: M a r k$ 's_force_field_parsing_code_requires_AmoebaOutOfPlaneBendForce

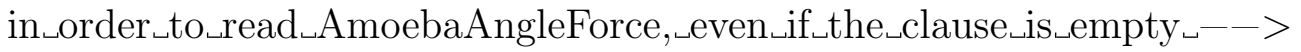

$<$ </AmoebaOutOfPlaneBendForce $>$

$\sim<$ AmoebaVdwForce $\_$type="BUFFERED $-14-7$ " radiusrule="CUBIC - MEAN"

radiustype $=" \mathrm{R}-\mathrm{MIN} "$ radiussize $="$ DIAMETER" $\_$epsilonrule $\left.=" \mathrm{HHG} "\right\lrcorner \mathrm{vdw}-13-$ scale $=" 0.0 " \mathrm{vdw}-14-$ scale $=" 1.0 " \mathrm{\nu dw}-15-$ scale $=" 1.0 ">$

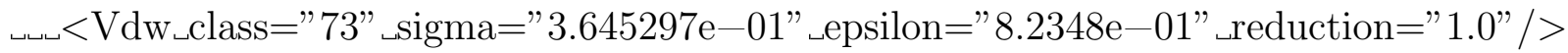

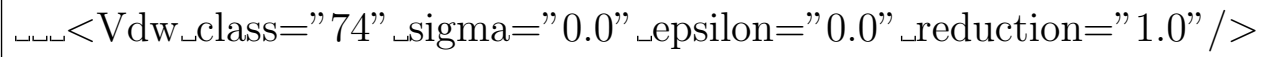

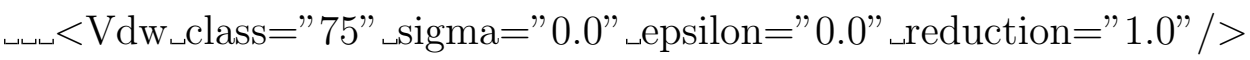

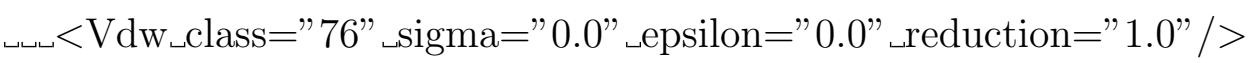

$\left\llcorner\left\llcorner<\mathrm{Vdw} \_\right.\right.$class $=" 77 "$ sigma $=" 0.0 " \_$epsilon $=" 0.0 "$ reduction $=" 1.0 " />$

$\left\llcorner \_<\right.$Vdw॰class $=" 78 "$ sigma $=" 0.0 " \_$epsilon="0.0" reduction=" $1.0 " />$

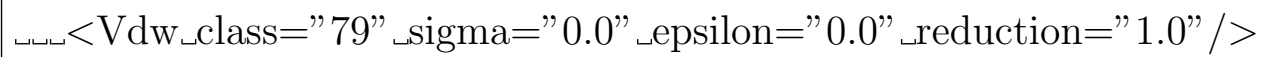

$\left\llcorner\left\llcorner<\mathrm{Vdw} \_\right.\right.$class $=" 80 "$ sigma $=" 0.0 "\llcorner$ epsilon $=" 0.0 "$ reduction $=" 1.0 " />$

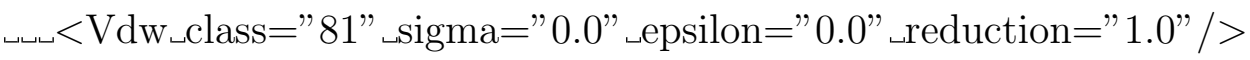

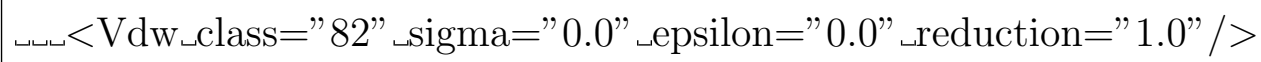

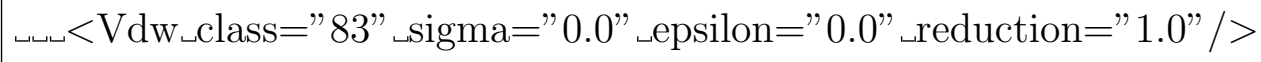

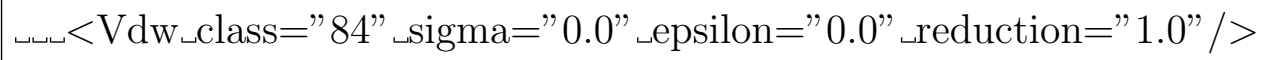

$\_</$AmoebaVdwForce $>$

$\sim \sim<$ AmoebaMultipoleForce_direct11Scale="0.0" $\lrcorner$ direct12Scale="1.0" $\lrcorner$ direct13Scale="1.0"

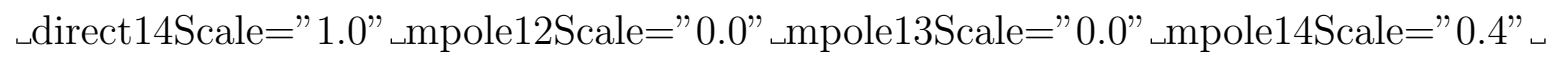

mpole15Scale="0.8" mutual11Scale="1.0" mutual12Scale="1.0" $\_$mutual13Scale

$=" 1.0 "$ mutual14Scale="1.0" $\lrcorner$ polar12Scale=" $0.0 "\lrcorner$ polar13Scale=" $0.0 "\lrcorner$ polar14Intra 
$=" 0.5 "$ polar14Scale $=" 1.0 "\lrcorner$ polar15Scale $=" 1.0 ">$

$\sim$ Multipole $\lrcorner$ type $=" 380 "\lrcorner c 0=" 0.0 "\lrcorner d 1=" 0.0 "\lrcorner d 2=" 0.0 "\lrcorner d 3=" 0.0 "\lrcorner q 11=" 0.0 "\lrcorner q 21=" 0.0 "$ $\llcorner\mathrm{q} 22=" 0.0 "\lrcorner \mathrm{q} 31=" 0.0 "\lrcorner \mathrm{q} 32=" 0.0 "\lrcorner \mathrm{q} 33=" 0.0 " />$

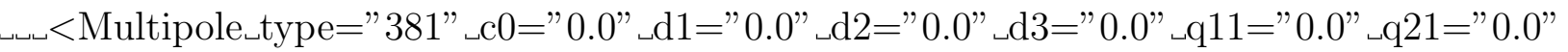

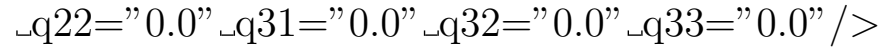

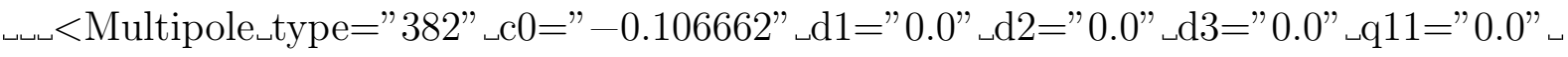
$\mathrm{q} 21=" 0.0 "\llcorner\mathrm{q} 22=" 0.0 "\llcorner\mathrm{q} 31=" 0.0 "\llcorner\mathrm{q} 32=" 0.0 "\llcorner\mathrm{q} 33=" 0.0 " />$ $<$ Multipole $\lrcorner$ type $=" 383 "\lrcorner c 0="-0.972581 "\lrcorner \mathrm{d} 1=" 0.0 "\lrcorner \mathrm{d} 2=" 0.0 "\lrcorner \mathrm{d} 3=" 0.0 "\llcorner\mathrm{q} 11=" 0.0 "\llcorner$ $\mathrm{q} 21=" 0.0 "\llcorner\mathrm{q} 22=" 0.0 "\llcorner\mathrm{q} 31=" 0.0 "\llcorner\mathrm{q} 32=" 0.0 "\llcorner\mathrm{q} 33=" 0.0 " />$ $<$ Multipole $\lrcorner$ type $=" 384 "\lrcorner c 0=" 0.599708 "\lrcorner \mathrm{d} 1=" 0.0 "\lrcorner \mathrm{d} 2=" 0.0 "\lrcorner \mathrm{d} 3=" 0.0 "\lrcorner \mathrm{q} 11=" 0.0 "\llcorner\mathrm{q} 21$ $=" 0.0 "\llcorner\mathrm{q} 22=" 0.0 "\llcorner\mathrm{q} 31=" 0.0 "\llcorner\mathrm{q} 32=" 0.0 "\llcorner\mathrm{q} 33=" 0.0 " />$

$<$ Multipole $\lrcorner$ type $=" 385 "\lrcorner c 0=" 0.828910 "\lrcorner \mathrm{d} 1=" 0.0 "\lrcorner \mathrm{d} 2=" 0.0 "\lrcorner \mathrm{d} 3=" 0.0 "\lrcorner \mathrm{q} 11=" 0.0 "\llcorner\mathrm{q} 21$ $=" 0.0 "\llcorner\mathrm{q} 22=" 0.0 "\llcorner\mathrm{q} 31=" 0.0 "\llcorner\mathrm{q} 32=" 0.0 "\llcorner\mathrm{q} 33=" 0.0 " />$ $<$ Multipole $\lrcorner$ type $=" 386 "\llcorner\mathrm{c} 0="-0.236929 "\lrcorner \mathrm{d} 1=" 0.0 "\lrcorner \mathrm{d} 2=" 0.0 "\lrcorner \mathrm{d} 3=" 0.0 "\llcorner\mathrm{q} 11=" 0.0 "$ ॰ $\mathrm{q} 21=" 0.0 "\llcorner\mathrm{q} 22=" 0.0 "\llcorner\mathrm{q} 31=" 0.0 "\llcorner\mathrm{q} 32=" 0.0 "\llcorner\mathrm{q} 33=" 0.0 " />$

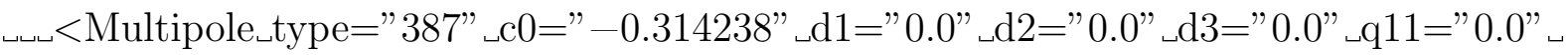
$\mathrm{q} 21=" 0.0 "\llcorner\mathrm{q} 22=" 0.0 "\llcorner\mathrm{q} 31=" 0.0 "\llcorner\mathrm{q} 32=" 0.0 "\llcorner\mathrm{q} 33=" 0.0 " />$ $<$ Multipole $\lrcorner$ type $=" 388 "\lrcorner \mathrm{c} 0=" 0.546572 "\lrcorner \mathrm{d} 1=" 0.0 "\lrcorner \mathrm{d} 2=" 0.0 "\lrcorner \mathrm{d} 3=" 0.0 "\lrcorner \mathrm{q} 11=" 0.0 "\lrcorner \mathrm{q} 21$ $=" 0.0 "\llcorner\mathrm{q} 22=" 0.0 "\llcorner\mathrm{q} 31=" 0.0 "\llcorner\mathrm{q} 32=" 0.0 "\lrcorner \mathrm{q} 33=" 0.0 " />$

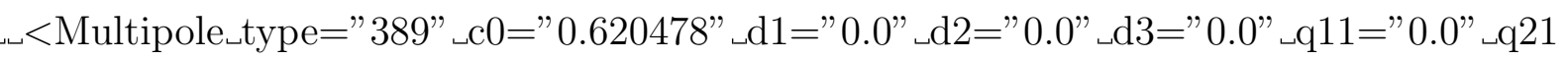
$=" 0.0 " \_q 22=" 0.0 "\left\llcorner q 31=" 0.0 " \_q 32=" 0.0 "\right\lrcorner q 33=" 0.0 " />$

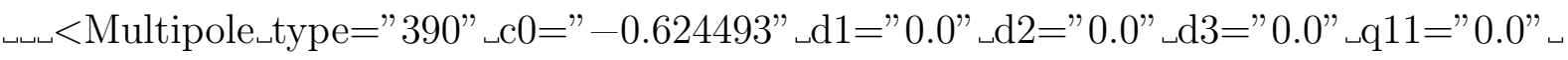
$\mathrm{q} 21=" 0.0 "\lrcorner \mathrm{q} 22=" 0.0 "$-q $31=" 0.0 "\llcorner\mathrm{q} 32=" 0.0 "\lrcorner \mathrm{q} 33=" 0.0 " />$

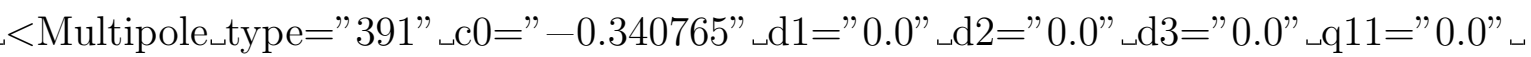
$\mathrm{q} 21=" 0.0 "\lrcorner \mathrm{q} 22=" 0.0 "\llcorner\mathrm{q} 31=" 0.0 "\llcorner\mathrm{q} 32=" 0.0 "\lrcorner \mathrm{q} 33=" 0.0 " />$ $<$ Polarize $\lrcorner$ type $=" 380 "$ "polarizability $=" 8.063631227791 \mathrm{e}-04 "$ "thole $=" 2.36164 \mathrm{e}-03 "$ 。

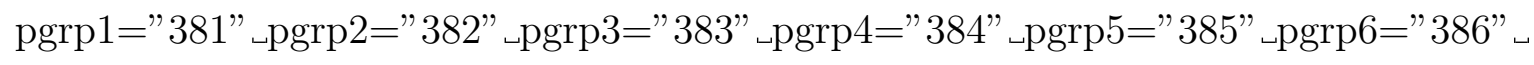


pgrp7=" 387" $\lrcorner \operatorname{pgrp} 8=" 388 "\lrcorner \operatorname{pgrp} 9=" 389 "\lrcorner \operatorname{pgrp} 10=" 390 "\lrcorner \operatorname{pgrp} 11=" 391 " />$

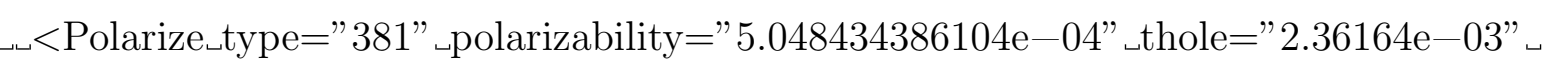

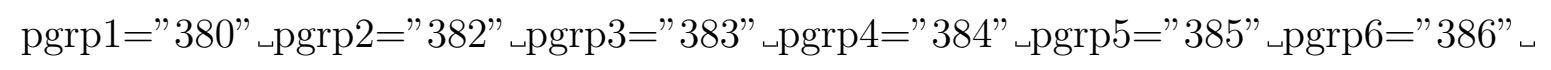

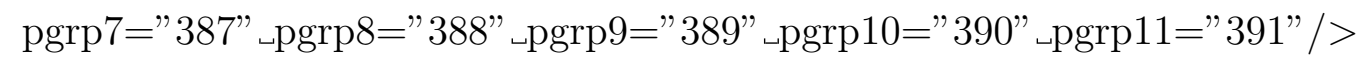

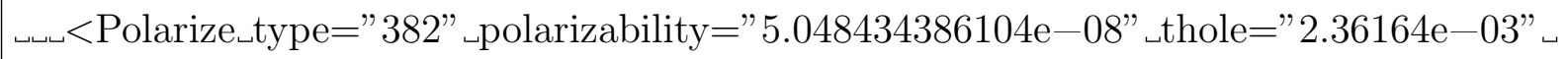

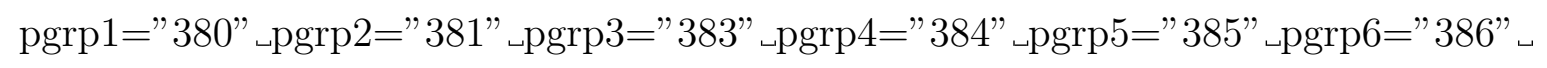

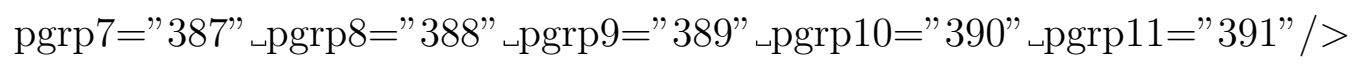

$<$ Polarize $\_$type $=" 383 "$ "polarizability $=" 5.048434386104 \mathrm{e}-08 "$.thole $=" 2.36164 \mathrm{e}-03 "$

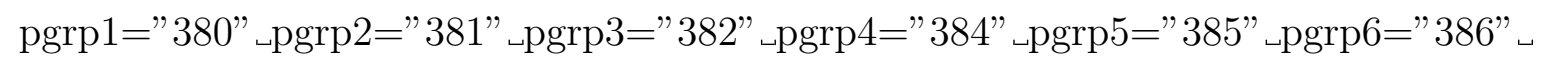

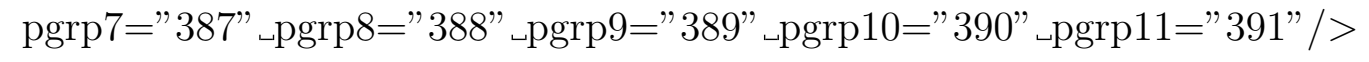

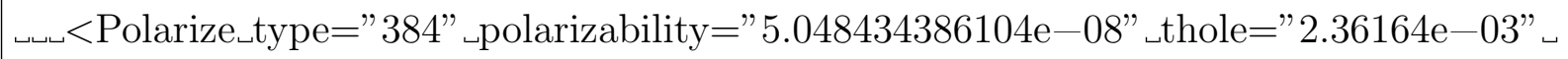

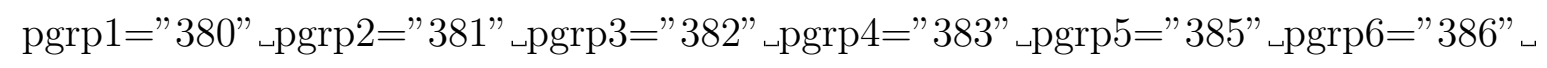

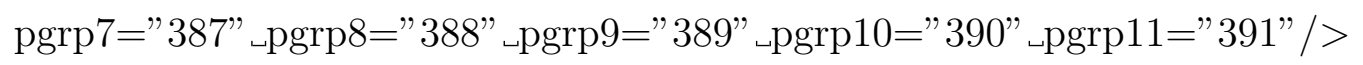

$\sim<$ Polarize $\lrcorner$ type $=" 385 "$ "polarizability $=" 5.048434386104 \mathrm{e}-08 "$ "thole $=" 2.36164 \mathrm{e}-03 "$

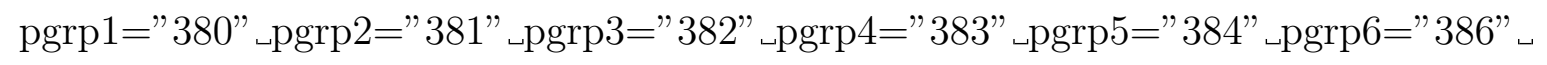

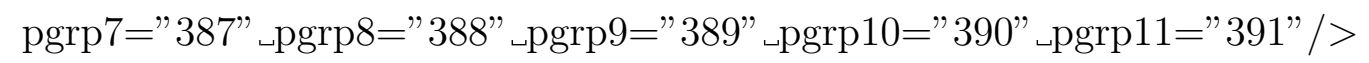

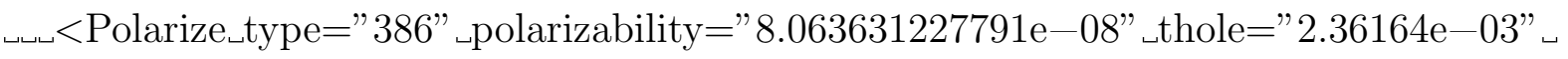

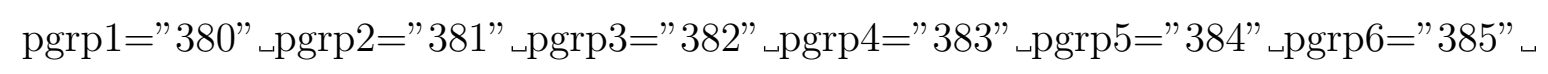

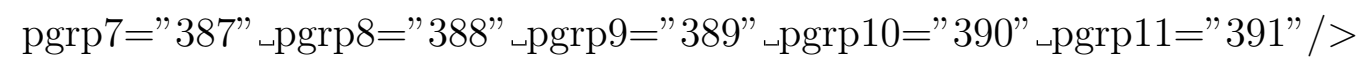

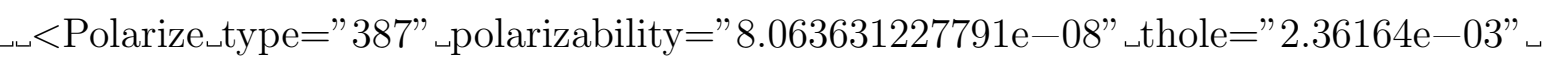

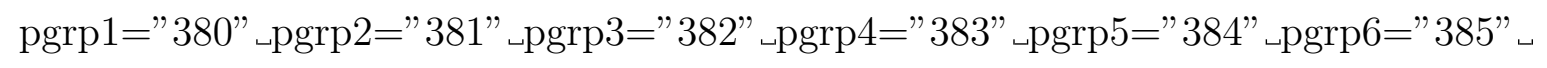

pgrp7="386" $\lrcorner$ pgrp8="388" $\lrcorner \operatorname{pgrp} 9=" 389 "\lrcorner \operatorname{pgrp} 10=" 390 "\lrcorner \operatorname{pgrp} 11=" 391 " />$

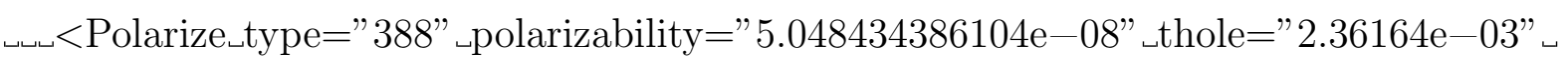

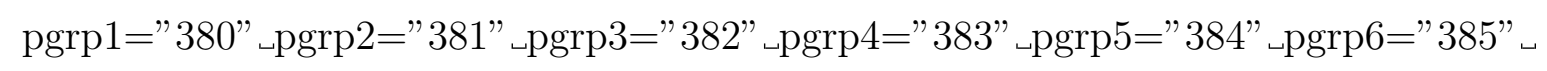

pgrp7="386" $\lrcorner \operatorname{pgrp} 8=" 387 "\lrcorner \operatorname{pgrp} 9=" 389 "\lrcorner \operatorname{pgrp} 10=" 390 "\lrcorner \operatorname{pgrp} 11=" 391 " />$

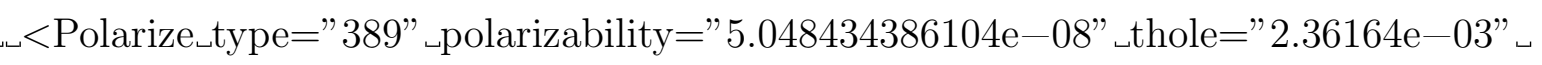

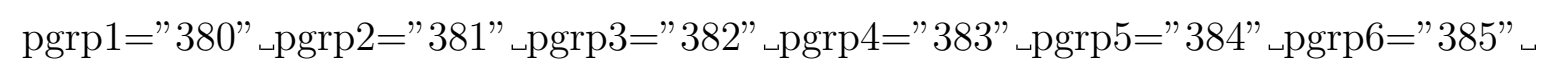




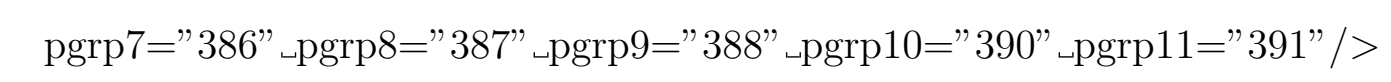

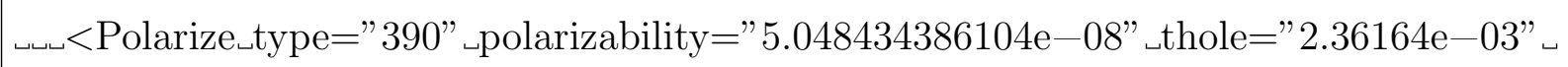

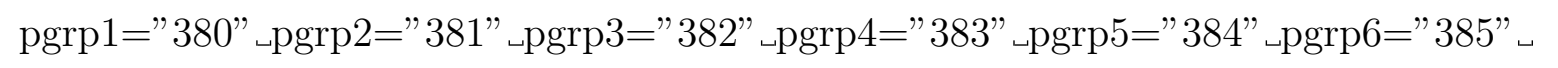

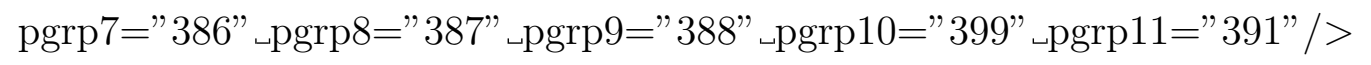

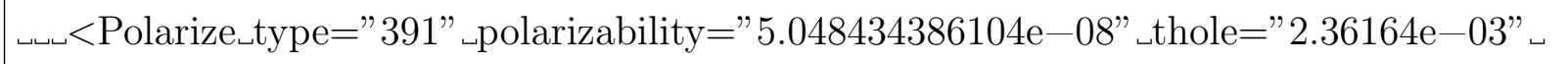

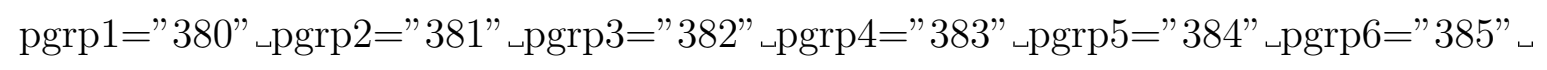

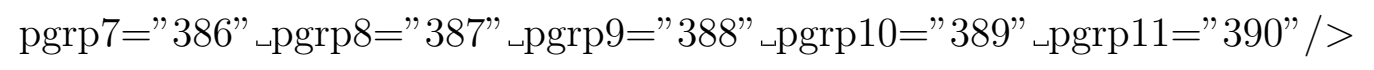

$\llcorner</$ AmoebaMultipoleForce $>$

$\backsim<$ AmoebaUreyBradleyForce $\_$cubic $=" 0.0 "$ "quartic="0.0" $>$

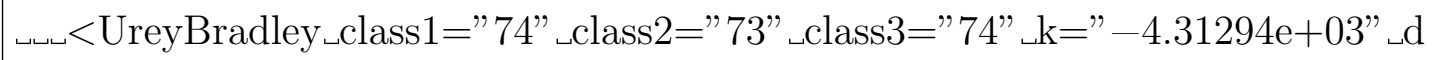

$$
=" 1.535676676685 \mathrm{e}-01 " />
$$

$\leftarrow$ /AmoebaUreyBradleyForce $>$

$\longleftarrow /$ ForceField $>$ 


\section{Impact of Broken Symmetry in MDCMs}

In this section the $\mathrm{H}_{2} \mathrm{O}$ dimer and cluster energies are compared before and after rotating all even-numbered monomers (i.e. the $2^{\text {nd }}, 4^{\text {th }}, 6^{\text {th }} \ldots$ ) by 180 degrees about their bisector in the PDB coordinate file. This effectively amounts to exchanging atom indices of the hydrogen atoms and quantifies the degree of symmetry-related differences in interaction energies. As MDCMs employ no symmetry constraints during fitting in the current work there will be a small discrepancy in interaction energy after rotating monomers. This effect should be minimal if the MDCM is sufficiently well fitted to the symmetric MEP around the molecule, but is non-existent in the multipolar and DCM models that share the spatial symmetry of the equilibrium structure of the molecule itself. The magnitude of the discrepancy is shown in Table S3. It should also be pointed out that even if the equilibrium structure of a molecule has a particular point-group symmetry and the distribution of charges shares this symmetry (whether for conventional PCs or for (M)DCM models), during an MD simulation the instantaneous geometries typically have lower symmetry.

The difference in $\mathrm{H}_{2} \mathrm{O}$ cluster electrostatic energies (including polarization) before and after rotating half of the monomers $\left(\Delta E_{\text {orig-rot }}\right)$ remains of the order of a few hundredths of a kcal/mol for all clusters and does not increase significantly with increasing cluster size. Together with results of bulk simulations, where all monomers are free to rotate their relative orientations during each trajectory, these results demonstrate that the spatial asymmetry in the MDCM charge arrangement does not have a significant impact on interaction energies or bulk simulation properties.

While development of symmetry constraints would eliminate this effect entirely, the fitting process would then be complicated. For example, charges may enter or leave the symmetry planes of the molecule during fitting. Each charge that leaves a plane must spawn an additional charge on the opposite side of that plane to maintain symmetry, favoring solutions with fewer in-plane charges as additional charges tend to improve the fit, and complicating 
Table S3: Comparison between multipolar and MDCM iAMOEBA electrostatic energies, including polarization. MDCM energies are calculated with all $\mathrm{H}_{2} \mathrm{O}$ monomers in their original orientations (orig), and with even-numbered monomers rotated 180 degrees about their bisector from their original orientations (rotated / rot) to evaluate the impact of symmetry violations in the MDCMs on cluster electrostatic energies. Energy differences are presented between MDCM monomers in their original orientations and their multipolar iAMOEBA equivalents $\left(\Delta E_{\text {orig-mtp }}\right)$, the same comparison is made using rotated monomers and multipolar iAMOEBA $\left(\Delta E_{\text {rot-mtp }}\right)$, and finally the energies of the original MDCM orientations are directly compared to MDCM energies with rotated monomers $\left(\Delta E_{\text {orig-rot }}\right)$. In the top part of the table energies for the 10 dimer structures are shown, while in the bottom part energies for various oligomers are presented.

\begin{tabular}{l|cccccc} 
Dimer & $\begin{array}{c}\text { iAMOEBA } \\
(\mathrm{kcal} / \mathrm{mol})\end{array}$ & $\begin{array}{c}\text { Original } \\
(\mathrm{kcal} / \mathrm{mol})\end{array}$ & $\begin{array}{c}\Delta E_{\text {orig-mtp }} \\
(\mathrm{kcal} / \mathrm{mol})\end{array}$ & $\begin{array}{c}\text { Rotated } \\
(\mathrm{kcal} / \mathrm{mol})\end{array}$ & $\begin{array}{c}\Delta E_{\text {rot-mtp }} \\
(\mathrm{kcal} / \mathrm{mol})\end{array}$ & $\begin{array}{c}\Delta E_{\text {orig-rot }} \\
(\mathrm{kcal} / \mathrm{mol})\end{array}$ \\
\hline 1 & -5.113 & -5.125 & -0.011 & -5.110 & 0.003 & 0.014 \\
2 & -4.505 & -4.465 & 0.040 & -4.491 & 0.013 & -0.027 \\
3 & -4.504 & -4.471 & 0.033 & -4.467 & 0.037 & 0.004 \\
4 & -3.824 & -3.836 & -0.011 & -3.814 & 0.011 & 0.022 \\
5 & -3.269 & -3.180 & 0.089 & -3.274 & -0.005 & -0.011 \\
6 & -2.972 & -2.863 & 0.109 & -2.990 & -0.018 & 0.020 \\
7 & -3.199 & -3.196 & 0.003 & -3.195 & 0.004 & 0.001 \\
8 & -1.572 & -1.587 & -0.014 & -1.577 & -0.005 & 0.009 \\
9 & -3.794 & -3.770 & 0.024 & -3.770 & 0.025 & 0.000 \\
10 & -3.016 & -3.026 & -0.010 & -3.025 & -0.009 & 0.001 \\
\hline Oligomer & iAMOEBA & Original & $\Delta E_{\text {orig-mtp }}$ & Rotated & $\Delta E_{\text {rot-mtp }}$ & $\Delta E_{\text {orig-rot }}$ \\
& $(\mathrm{kcal} / \mathrm{mol})$ & $(\mathrm{kcal} / \mathrm{mol})$ & $(\mathrm{kcal} / \mathrm{mol})$ & $(\mathrm{kcal} / \mathrm{mol})$ & $(\mathrm{kcal} / \mathrm{mol})$ & $(\mathrm{kcal} / \mathrm{mol})$ \\
\hline trimer & -13.770 & -13.782 & -0.012 & -13.725 & 0.045 & 0.056 \\
tetramer & -24.529 & -24.436 & 0.093 & -24.457 & 0.071 & -0.022 \\
pentamer & -32.321 & -32.266 & 0.055 & -32.180 & 0.141 & 0.086 \\
hexamer & -41.459 & -41.348 & 0.111 & -41.262 & 0.197 & 0.085 \\
heptamer & -51.299 & -51.129 & 0.171 & -51.009 & 0.290 & 0.119 \\
octamer & -64.672 & -64.238 & 0.434 & -64.166 & 0.506 & 0.072 \\
nonamer & -72.896 & -72.478 & 0.419 & -72.424 & 0.472 & 0.054 \\
decamer & -82.858 & -82.402 & 0.456 & -82.313 & 0.544 & 0.089 \\
& & & & & &
\end{tabular}


control over the total number of charges in the fit. While modifications can be envisaged to deal with such issues, the fitting process is likely to become more complex as a result. As such modifications are additionally applicable to a relatively small subset of available molecules (those with high symmetry) they are currently left as a future development.

For the purpose of the current study, namely demonstrating the accuracy and performance of new developments to MDCMs with respect to existing multipolar and TIP $n \mathrm{P}$ force field models, the results in Table S3 and the results section of the main text show that symmetry constraints are not required. 


\section{O...O Radial Distribution Functions}

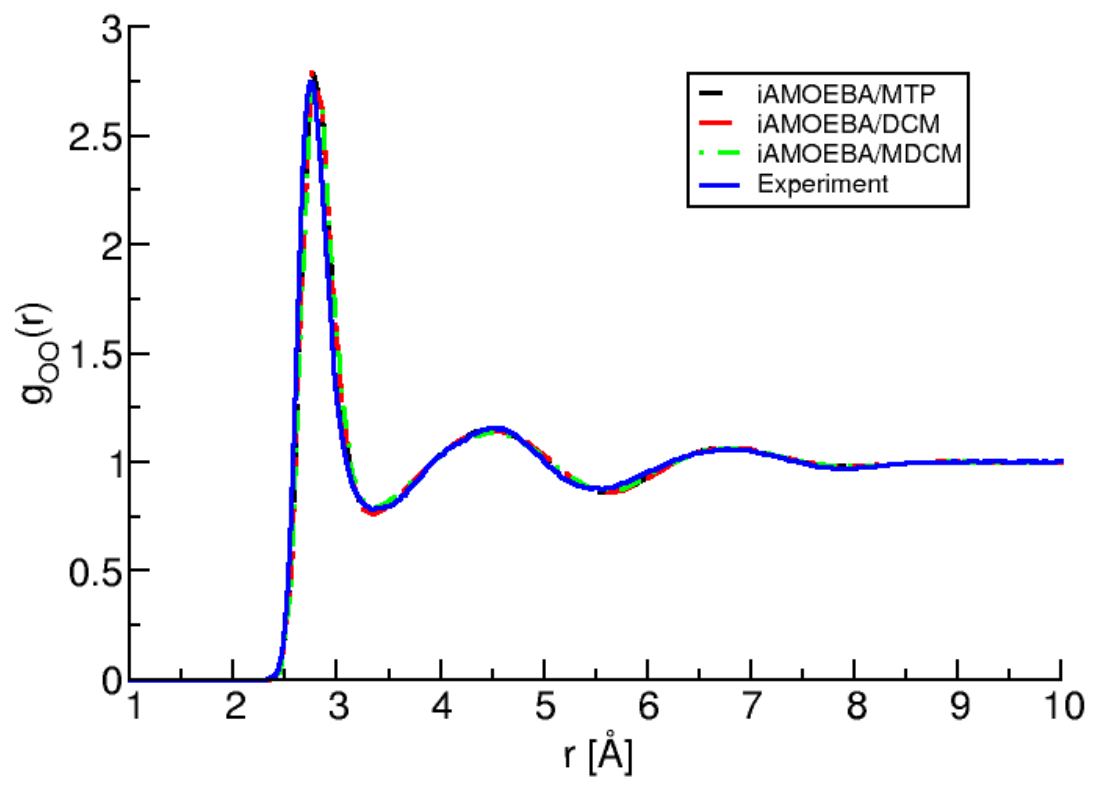

Figure S1: Oxygen-oxygen radial distribution functions for liquid water at $298.15 \mathrm{~K}$ and 1 atm. DCM and MDCM implementations are compared with results using the original, multipolar water iAMOEBA water model and with experimental neutron diffraction data. ${ }^{\text {S3 }}$ 


\section{O...H Radial Distribution Functions}

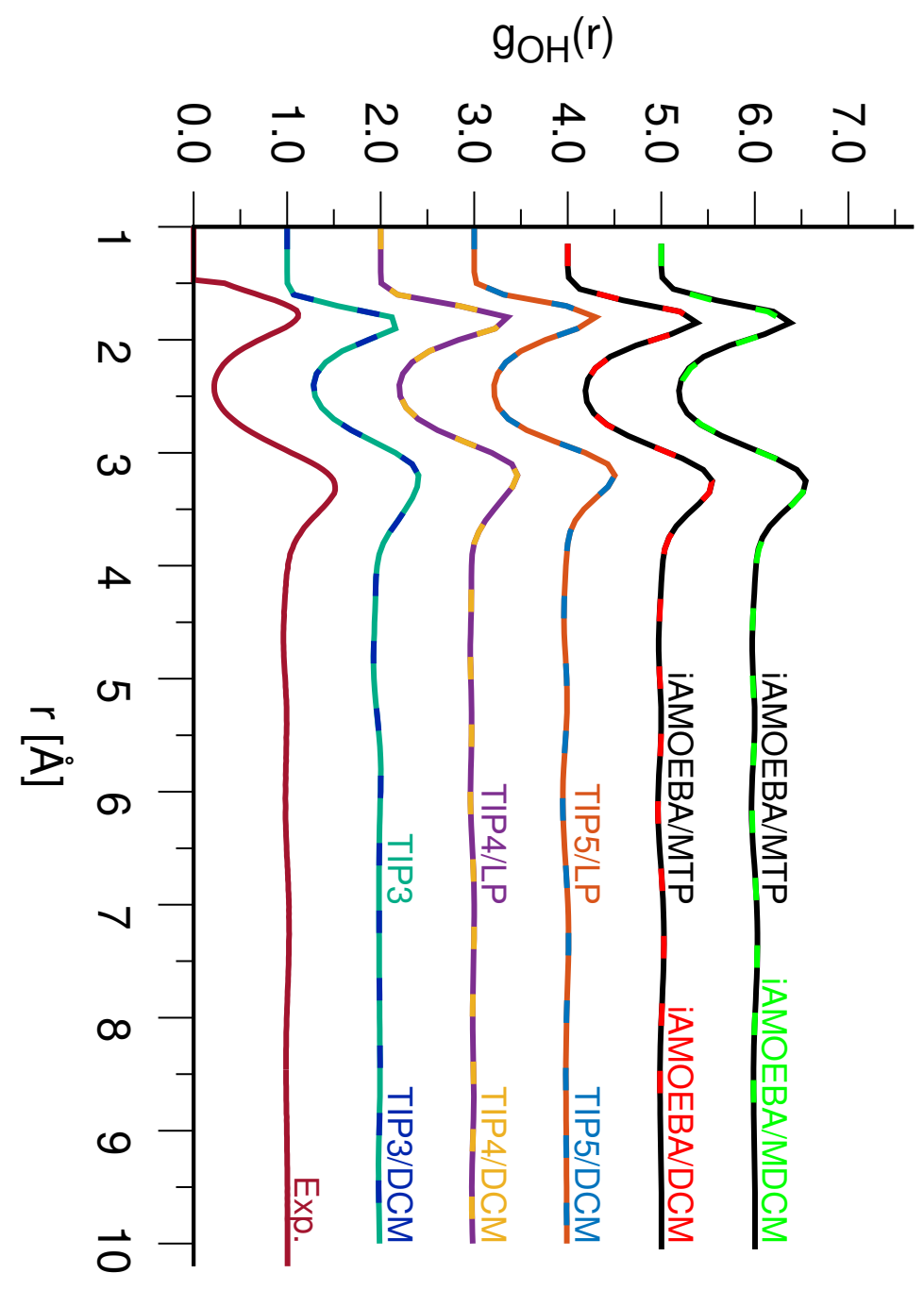

Figure S2: Oxygen-hydrogen radial distribution functions for liquid water at $298.15 \mathrm{~K}$ and $1 \mathrm{~atm}$. Various simulation methods are compared against experimental Neutron diffraction data. ${ }^{\text {S3 }}$ Successive curves are offset 1 unit along the $y$-axis for clarity. 


\section{$9 \quad$ H...H Radial Distribution Functions}

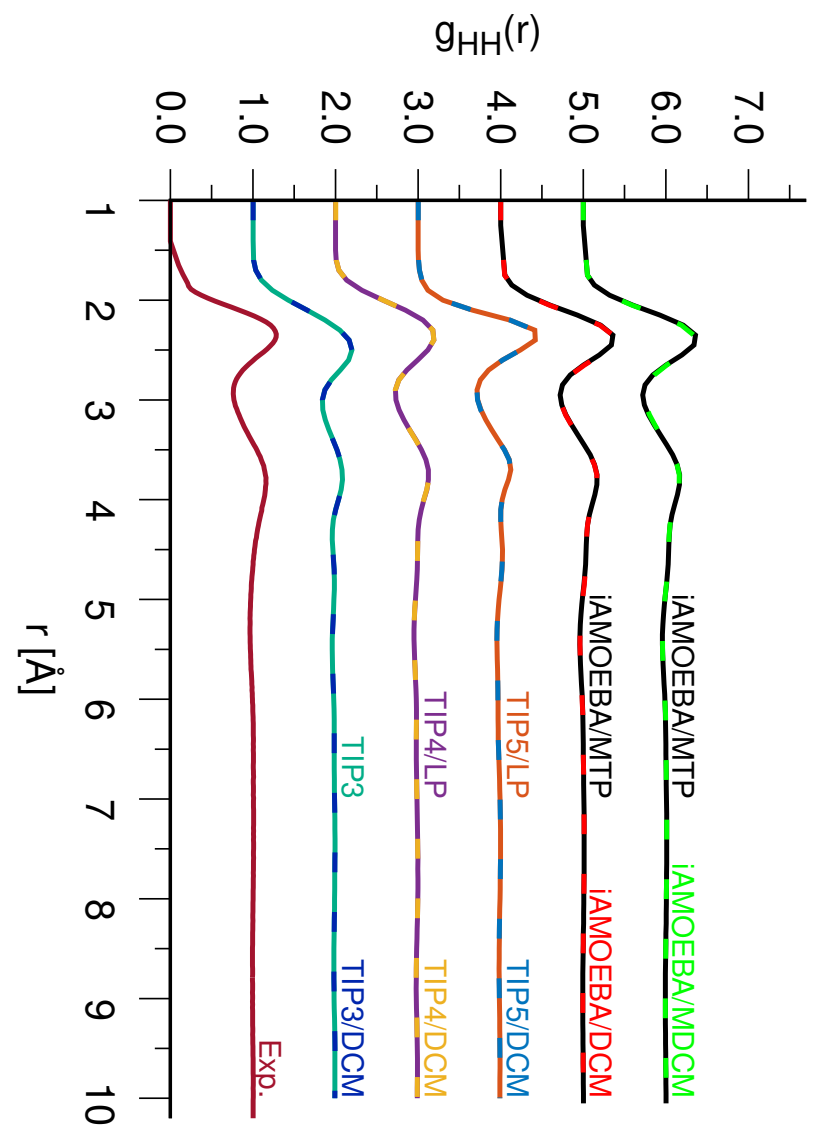

Figure S3: Hydrogen-hydrogen, radial distribution functions for liquid water at $298.15 \mathrm{~K}$ and $1 \mathrm{~atm}$. Various simulation methods are compared against experimental Neutron diffraction data. ${ }^{\text {S3 }}$ Successive curves are offset 1 unit along the $y$-axis for clarity. 


\section{Computational Efficiency}

As indicated in section 1, using (M)DCM over MTPs may also have a beneficial effect on computational efficiency in MD simulations. It is first noted that both approaches scale fundamentally as $O(N)$ or $O(N \log N)$ where $N$ is the number of atoms ${ }^{\mathrm{S} 4}$ as the number of interacting atoms remains the same in both cases, and the multiple charges or multipole terms belonging to each atom do not interact with one another. The relative number of terms for MDCM compared with MTP for a basic implementation using a spherical tensor formalism for the multipole expansion can be gleaned from Equations 2-11.

The cost for both, distributed charges and multipole moments, therefore arises from the increased number of terms per atom-atom interaction. For $n$ non-zero multipole terms and $n$ distributed charges each atom-atom interaction involves $n(n+1) / 2$ component terms. As multipole models allow for truncation at different ranks for different atoms, ${ }^{\mathrm{S} 5}$ and MDCMs allow for different numbers of charges per atom the general summation over terms per atomatom interaction is

$$
V_{\mathrm{ab}}\left(\mathbf{r}_{\mathrm{ab}}\right)=\sum_{i=1}^{n_{\mathrm{a}}} \sum_{j=1}^{n_{\mathrm{b}}} V_{i j}\left(\mathbf{r}_{\mathrm{ab}}\right)
$$

where $n_{\mathrm{a}}$ is the number of nonzero multipole moment components or distributed charges on atom $a$, respectively. $V_{i j}\left(\mathbf{r}_{\mathrm{ab}}\right)$ is the contribution to the interaction energy $V_{\mathrm{ab}}\left(\mathbf{r}_{\mathrm{ab}}\right)$ between atoms $a$ and $b$ from multipole moment components or charges $i$ and $j$ as a function of atomic separation $\mathbf{r}_{\mathrm{ab}}$ for both models, including the relative orientations of the local axis systems for multipolar models. Computational cost is therefore fundamentally linked to $n_{\mathrm{a}}, n_{\mathrm{b}}$ and the functional form of $V_{i j}\left(\mathbf{r}_{\mathrm{ab}}\right)$.

Hence, substantial computational savings can be achieved by reducing the number of terms $n_{\mathrm{a}}$ and $n_{\mathrm{b}}$, see Equation 18. For multipole moments this is typically achieved by diagonalizing the Cartesian quadrupole moment tensor and converting to spherical polar coordinates, leaving maximally 6 non-zero multipole moment components. In highly symmetric systems 
this number may reduce further, ${ }^{\mathrm{S} 5}$ and the multipole expansion may be truncated at lower rank for certain atom types such as hydrogen atoms. In MDCMs the fitting algorithm is explicitly conceived to use as few charges per atom as possible. In the current study it was demonstrated that no more than 10 charges are required in total compared to 19 non-zero multipole moment components in the iAMOEBA model. For PhF, 22 charges were used for the entire molecule (see Figure ?? of the main text) compared with 46 non-zero multipole components.

Next, the cost of evaluating the coordinate-dependent terms $V_{i j}\left(\mathbf{r}_{\mathrm{ab}}\right)$ is considered. A comparison of these terms has been presented previously ${ }^{\mathrm{S} 2}$ but is nevertheless discussed in some detail here.

- While the use of off-centered charges introduces complexity in evaluation of local axes to place the charges, these terms are the same for all atom-atom interactions and are evaluated once per atom per simulation time step. For multipoles the local axes also need to be evaluated to determine the torques.

- Conversely, each Coulomb charge-charge term or multipole--multipole term and its derivative must be evaluated for every atom-atom interaction and so dominates the computational cost. For multipole moments higher ranking terms incur increasing computational cost due to the need to evaluate trigonometric functions (Equations $2-11$ ), although schemes exist to reduce this. ${ }^{\mathrm{S} 6}$ For MDCM there is a small increase in complexity of the forces with respect to an atom-centered charge model to include the axis derivatives that are calculated once per time step, but as shown in Equation 18 the form of the interaction energy is the same as for an atom-centered charge model.

- For MDCM an additional inverse square root operation to evaluate $1 / r$ for each offnuclear charge position is incurred, whereas all multipole moments share the same local origin for a given atom and thus share the same value of $1 / r$. 
The comparison of computational cost of $V_{i j}\left(\mathbf{r}_{\mathrm{ab}}\right)$ is thus primarily a comparison of the cost of the additional inverse square root operation per interaction ij (MDCM), with the significantly increased number of multiplication and division operations and the trigonometric terms required especially for higher order multipole terms. Based on this argument it also becomes clear that in order to increase the accuracy of a multipolar model beyond rank $l=2$ (quadrupole moment) a significant cost will be incurred due to the large increase in $n_{\mathrm{a}}$ and $n_{\mathrm{b}}$ and the additional increase in complexity of $V_{i j}\left(\mathbf{r}_{\mathrm{ab}}\right)$, whereas additional charges can be added one by one in MDCM with no increase in complexity of $V_{i j}\left(\mathbf{r}_{\mathrm{ab}}\right)$.

The following timings are illustrative examples for two particular implementations for two different systems used in the current work and caution should be used before drawing general conclusions (see further below). The first system studied was the iAMOEBA waterbox introduced in the main text. To make a meaningful comparison the polarization term was removed, as current OpenMM functionality required 13 multipolar, polarizable sites to be created for MDCM where only 3 polarizable sites (at the nuclear positions) and no multipole moments would have been necessary with a minor modification to the code (see also Section ?? of the main text). The polarization energy therefore dominated the calculation time and hindered analysis of the cost of the underlying charge model. To remove polarization the Multipole definitions of the OpenMM parameter file (section 5) were replace by "NonbondedForce" definitions of the form:

$$
<\text { Atom name="M1" type="382" charge=" }-0.106662 " \text { sigma="0.0" epsilon="0.0" } />
$$

To keep results comparable, polarizabilities were also zeroed when using multipoles, and $10^{5}$ time steps (50 ps) equilibration was run with PME for each model (see section ??-?? of the main text for simulation details). The initial potential energies for the simulation water box without polarization are within $0.3 \mathrm{kcal} / \mathrm{mol}$ for both electrostatic models $(-688.5$ $\mathrm{kcal} / \mathrm{mol}$ for MDCM vs. $-688.8 \mathrm{kcal} / \mathrm{mol}$ for MTP). The simulation wall clock time on a single NVidia Titan Xp GPU with CUDA 10.2 and Intel(R) Xeon(R) E5-2620 v4 CPU was 
$97.8 \mathrm{~s}$ for MDCM (averaged over 5 runs) and $112.5 \mathrm{~s}$ for MTPs, i.e. a speedup of $\sim 15 \%$ for MDCM compared with MTP. It should be emphasized that the code in OpenMM is not likely to be optimized for MDCM, as models with more than one or two off-centered charges are currently uncommon, and even the non-polarizable OpenMM MDCM implementation leaves 10 superfluous van der Waals sites (1 per off-center charge) that are not required by MDCM, and 3 superfluous charged sites at the nuclear positions, while more focus on the implementation of virtual sites could potentially lead to further gains. In the same way the iAMOEBA multipolar implementation with zeroed polarizabilities would be made more efficient by completely removing the polarizable sites, which appear to incur a computational cost even when polarizabilities are zeroed. Despite these caveats it is encouraging that the MDCM representation runs efficiently, even without the minor modifications to the simulation code suggested.

A similar comparison was run for bulk PhF with spherical cut-offs in CHARMM for 4000 time steps (4 ps) heating and 4000 time steps (4 ps) equilibration of the pure liquid $\mathrm{PhF}$ simulation box described in section ?? of the main text with 8 CPU cores of an Intel Xeon E5-2630 v4 CPU. In this case neither code has been heavily optimized, but the average wall clock time averaged over 5 simulations was 848 s (MDCM) vs. 3233 s (MTP). Again, it is gratifying to note the decreased computational cost associated with the MDCM, but again both timings describe one possible implementation of each algorithm in one particular hardware and software environment for the PhF system studied here, and caution should still be used before drawing general conclusions.

The discussion to this point neglects details of the algorithms, implementation, hardware, compilers and others that can make a decisive difference to the efficiency for large scale applications. Control and consideration of all of these points for both approaches will be required for further quantitative comparison of computational cost, and are beyond the scope of the current analysis. 


\section{References}

(S1) Stone, A. J. The Theory of Intermolecular Forces; Oxford University Press: Oxford, U.K., 2000.

(S2) Devereux, M.; Raghunathan, S.; Fedorov, D. G.; Meuwly, M. A Novel, computationally efficient multipolar model employing distributed charges for molecular dynamics simulations. J. Chem. Theory Comput. 2014, 10, 4229-4241.

(S3) Soper, A. The radial distribution functions of water and ice from 220 to $673 \mathrm{~K}$ and at pressures up to $400 \mathrm{MPa}$. Chem. Phys. 2000, 258, 121-137.

(S4) Nilsson, L. Efficient table lookup without inverse square roots for calculation of pair wise atomic interactions in classical simulations. jcc 2009, 30, 1490-1498.

(S5) Kramer, C.; Gedeck, P.; Meuwly, M. Atomic multipoles: Electrostatic potential fit, local reference axis systems, and conformational dependence. J. Comput. Chem. 2012, 33, 1673-1688.

(S6) Wu, X.; Pickard, F. C.; Brooks, B. R. Isotropic periodic sum for multipole interactions and a vector relation for calculation of the Cartesian multipole tensor. jcp 2016, 145 , 164110. 